\title{
A Specification Test for Nonlinear Nonstationary Models
}

\author{
Qiying Wang \\ University of Sydney \\ Peter C. B. Phillips \\ Yale University, University of Auckland, \\ University of Southampton $\& 5$ Singapore Management University
}

January 31, 2012

This supplement gives the proofs of Theorems 5.1-5.3 (see Section 7), the proofs of Propositions 6.1-6.8 (see Section 8), and additional simulation results (see Section 9) in the official publication. Results (formulae) cited are along the lines of the official publication.

\section{Proofs of Theorems 5.1-5.3}

As usual, let $C, C_{1}, C_{2}, \ldots$ be constants that may differ at each appearance. In order to prove Theorems 5.1-5.3, we first claim that, for any finite $t, x, L_{G}(t, x)$ can be defined as an $L_{2}$ limit of $L_{G}^{N}(t, x):=L_{G}^{-N, N}(t, x)$ as $N \rightarrow \infty$, where

$$
L_{G}^{M, N}(t, x)=\frac{1}{2 \pi} \int_{M}^{N} e^{-i u x} \int_{0}^{t} \int_{0}^{t} e^{i u\left[G(s)-G\left(s_{1}\right)\right]} d s d s_{1} d u .
$$

In this regard, it suffices to show that, for any fixed $t, x$,

$$
E\left|L_{G}^{M, N}(t, x)\right|^{2} \rightarrow 0
$$

as $M, N \rightarrow \infty$. Indeed, the result (7.2) implies the $L_{2}$ convergence of $L_{G}^{N}(t, x)$. Hence, in the framework of $L_{2}$-theory, we have

$$
\begin{aligned}
\lim _{N \rightarrow \infty} L_{G}^{N}(t, x) & =\frac{1}{2 \pi} \int_{-\infty}^{\infty} e^{-i u x} \int_{0}^{t} \int_{0}^{t} e^{i u\left(G(s)-G\left(s_{1}\right)\right.} d s d s_{1} d u \\
& =\frac{1}{2 \pi} \int_{-\infty}^{\infty} e^{-i u x} \int_{-\infty}^{\infty} e^{i u y} L_{G}(t, y) d y d u \\
& =\int_{-\infty}^{\infty} L_{G}(t, y) d y \frac{1}{2 \pi} \int_{-\infty}^{\infty} e^{i u(y-x)} d u \\
& =L_{G}(t, x),
\end{aligned}
$$


which implies the claim. To prove (7.2), note that

$$
\begin{aligned}
E\left|L_{G}^{M, N}(t, x)\right|^{2} & =\frac{1}{(2 \pi)^{2}} \int_{M}^{N} \int_{M}^{N} e^{i\left(u_{1}-u\right) x} \int_{0}^{t} \int_{0}^{t} \int_{0}^{t} \int_{0}^{t} E e^{i u\left[G(s)-G\left(s_{1}\right)\right]-i u_{1}\left[G\left(s_{2}\right)-G\left(s_{3}\right)\right]} \\
& \leq C \sum_{\substack{i_{j} \in\{1,2,3,4\} \\
i_{1} \neq i_{2} \neq i_{3} \neq i_{4}}} \int_{M}^{N} \int_{M}^{N} \int_{s_{1}} d s_{2} d s_{3} d u d u_{1}
\end{aligned}
$$

where, with $f_{i_{1}, i_{2}, i_{3}, i_{4}}\left(u, u_{1}\right)=E e^{i u\left[G\left(x_{i_{1}}\right)-G\left(x_{i_{2}}\right)\right]-i u_{1}\left[G\left(x_{i_{3}}\right)-G\left(x_{i_{4}}\right)\right]}$,

$$
\Pi_{i_{1}, i_{2}, i_{3}, i_{4}}\left(u, u_{1}\right)=\int \ldots \int_{0<x_{1}<x_{2}<x_{3}<x_{4}<t}\left|f_{i_{1}, i_{2}, i_{3}, i_{4}}\left(u, u_{1}\right)\right| d x_{1} d x_{2} d x_{3} d x_{4} .
$$

The result (7.2) will follow if each term in the right hand of (7.3) converges to 0, as $M, N \rightarrow \infty$. Only consider $i_{1}=4, i_{3}=3, i_{2}=2, i_{4}=1$, and without loss of generality, assume $t=1$. All others are similar except more simpler. Note that, for any $s_{1} \leq s_{2}$,

$$
G\left(s_{2}\right)=\int_{0}^{s_{2}} e^{\kappa\left(s_{2}-u\right)} d W(u)=e^{\kappa\left(s_{2}-s_{1}\right)} G\left(s_{1}\right)+\int_{s_{1}}^{s_{2}} e^{\kappa\left(s_{2}-u\right)} d W(u)
$$

Write $G^{*}\left(s_{1}, s_{2}\right)=\int_{s_{1}}^{s_{2}} e^{\kappa\left(s_{2}-u\right)} d W(u)$. Simple calculations show that $G^{*}\left(s_{1}, s_{2}\right) \sim N\left(0, \sigma_{s_{1} s_{2}}^{2}\right)$ where $\sigma_{s_{1} s_{2}}^{2}=\int_{s_{1}}^{s_{2}} e^{2 \kappa\left(s_{2}-u\right)} d u \geq \gamma_{0}\left(s_{2}-s_{1}\right)$ for some $\gamma_{0}>0$. This, together with the independence between $G\left(s_{1}\right)$ and $G^{*}\left(s_{1}, s_{2}\right)$, yields that, for $0<x_{1}<x_{2}<x_{3}<x_{4} \leq 1$

$$
\begin{aligned}
\left|f_{4,2,3,1}\left(u, u_{1}\right)\right| & =\left|E \exp \left\{i u G\left(x_{4}\right)-i u_{1} G\left(x_{3}\right)-i u G\left(x_{2}\right)+i u_{1} G\left(x_{1}\right)\right\}\right| \\
& =\left|E \exp \left\{i u G^{*}\left(x_{3}, x_{4}\right)+i\left(u e^{\kappa\left(x_{4}-x_{3}\right)}-u_{1}\right) G\left(x_{3}\right)-i u G\left(x_{2}\right)+i u_{1} G\left(x_{1}\right)\right\}\right| \\
& \leq\left|E \exp \left\{i u G^{*}\left(x_{3}, x_{4}\right)+i\left(u e^{\kappa\left(x_{4}-x_{3}\right)}-u_{1}\right) G^{*}\left(x_{2}, x_{3}\right)\right\}\right| \\
& \leq \exp \left\{-\gamma_{0} u^{2}\left(x_{4}-x_{3}\right) / 2-\gamma_{0}\left(u e^{\kappa\left(x_{4}-x_{3}\right)}-u_{1}\right)^{2}\left(x_{3}-x_{2}\right) / 2\right\} .
\end{aligned}
$$

Hence it is readily seen that

$$
\begin{aligned}
& \int_{M}^{N} \int_{M}^{N} \Pi_{4,2,3,1}\left(u, u_{1}\right) d u d u_{1} \\
\leq & \int_{M}^{N} \int_{M}^{N} \int_{0}^{1} \int_{0}^{1} \exp \left\{-\gamma_{0} u^{2} x / 2-\gamma_{0}\left(u e^{\kappa x}-u_{1}\right)^{2} y / 2\right\} d x d y d u d u_{1} \\
\leq & \int_{M}^{N} \int_{0}^{1} \exp \left\{-\gamma_{0} u^{2} x / 2\right\} d x d u \int_{-\infty}^{\infty} \int_{0}^{1} \exp \left\{-\gamma_{0} u_{1}^{2} y / 2\right\} d y d u_{1} \\
\leq & C \int_{M}^{N} u^{-2} d u \rightarrow 0,
\end{aligned}
$$

as $M, N \rightarrow \infty$, as required. 
Proof of Theorem 5.1. We first prove the results (5.6) and (5.7) under an additional condition:

Con: $\quad g(x)$ is continuous and $\hat{g}(t)$ has a compact support,

where $\hat{g}(x)=\int_{-\infty}^{\infty} e^{i x t} g(t) d t$.

To start, noting that $g(x)=\frac{1}{2 \pi} \int_{-\infty}^{\infty} e^{i t x} \hat{g}(-t) d t$, we have

$$
\begin{aligned}
\frac{c_{n}}{n^{2}} \sum_{k, j=1}^{[n r]} g\left[c_{n}\left(x_{k, n}-x_{j, n}\right)\right] & =\frac{1}{2 \pi n^{2}} \sum_{k, j=1}^{[n r]} \int_{-\infty}^{\infty} \hat{g}\left(-s / c_{n}\right) e^{i s\left(x_{k, n}-x_{j, n}\right)} d s \\
& =R_{1 n}(r)+R_{2 n}(r),
\end{aligned}
$$

where, for some $A>0$,

$$
\begin{aligned}
& R_{1 n}(r)=\frac{1}{2 \pi n^{2}} \sum_{k, j=1}^{[n r]} \int_{|s| \leq A} \hat{g}\left(-s / c_{n}\right) e^{i s\left(x_{k, n}-x_{j, n}\right)} d s \\
& R_{2 n}(r)=\frac{1}{2 \pi n^{2}} \sum_{k, j=1}^{[n r]} \int_{|s|>A} \hat{g}\left(-s / c_{n}\right) e^{i s\left(x_{k, n}-x_{j, n}\right)} d s .
\end{aligned}
$$

Furthermore, $R_{1 n}(r)$ can be written as

$$
R_{1 n}(r)=\frac{1}{2 \pi} \int_{|s| \leq A} \hat{g}\left(-s / c_{n}\right) \int_{0}^{r} \int_{0}^{r} e^{i s\left(x_{[n u], n}-x_{[n v], n}\right)} d u d v d s+o_{P}(1) .
$$

Recall $c_{n} \rightarrow \infty$. It is readily seen that $\sup _{|s| \leq A}\left|\hat{g}\left(-s / c_{n}\right)-\hat{g}(0)\right| \rightarrow 0$ for any fixed $A>0$. Hence, by recalling (5.2), it follows from the continuous mapping theorem that, for any $A>0$ and fixed $r$,

$$
R_{1 n}(r) \rightarrow_{D} \frac{\hat{g}(0)}{2 \pi} \int_{|s| \leq A} \int_{0}^{r} \int_{0}^{r} e^{i s[G(u)-G(v)]} d u d v d s
$$

as $n \rightarrow \infty$. On the other hand, under the same probability space used in (5.3), we have

$$
\begin{aligned}
\sup _{0 \leq r \leq 1} \mid & R_{1 n}(r)-\frac{\hat{g}(0)}{2 \pi} \int_{|s| \leq A} \int_{0}^{r} \int_{0}^{r} e^{i s\left[G_{1}(u)-G_{1}(v)\right]} d u d v d s \mid \\
\leq & \frac{1}{2 \pi} \int_{|s| \leq A}\left|\hat{g}\left(-s / c_{n}\right)-\hat{g}(0)\right| d s \\
& \quad+\frac{|\hat{g}(0)|}{2 \pi} \int_{|s| \leq A} \int_{0}^{1} \int_{0}^{1}\left|e^{i s\left(x_{[n u], n}-G_{1}(u)+x_{[n v], n}-G_{1}(v)\right)}-1\right| d u d v d s \\
\leq & o(1)+O(1) \sup _{0 \leq u \leq 1}\left|x_{[n u], n}-G_{1}(u)\right|=o_{P}(1) .
\end{aligned}
$$


By (7.6) and (7.7), noting that $\hat{g}(0)=\omega$ and, as $A \rightarrow \infty, \frac{1}{2 \pi} \int_{|s| \leq A} \int_{0}^{r} \int_{0}^{r} e^{i s[G(u)-G(v)]} d u d v d s$ converges to $L_{G}(r, 0)$ in $L_{2}$ uniformly on $r \in[0,1]$, the results (5.6) and (5.7) will follow under the additional condition (7.4), if we prove

$$
\sup _{0 \leq r \leq 1} E\left|R_{2 n}(r)\right|^{2} \rightarrow 0
$$

as $n \rightarrow \infty$ first and then $A \rightarrow \infty$.

In order to prove (7.8), we need some preliminaries. Write $\eta_{r}^{\prime}=\sum_{q=1}^{r} \epsilon_{q} \phi_{r-q}$ and

$$
y_{\lambda, n}^{\prime}=\sum_{r=1}^{\lambda} \rho^{\lambda-r} \eta_{r}^{\prime}=\sum_{q=1}^{\lambda} \epsilon_{q} \sum_{r=0}^{\lambda-q} \rho^{\lambda-q-r} \phi_{r}=\sum_{q=1}^{\lambda} \epsilon_{q} a(\lambda-q),
$$

where $a(v)=\sum_{r=0}^{v} \rho^{v-r} \phi_{r}$. Simple calculations show that, whenever $k \geq j \geq l \geq m$,

$$
\begin{aligned}
& s\left(y_{k, n}^{\prime}-y_{j, n}^{\prime}\right)-t\left(y_{l, n}^{\prime}-y_{m, n}^{\prime}\right) \\
& =s \sum_{q=j+1}^{k} \epsilon_{q} a(k-q)+s \sum_{q=l+1}^{j} \epsilon_{q}[a(k-q)-a(j-q)] \\
& \quad+\sum_{q=m+1}^{l} \epsilon_{q}\{s[a(k-q)-a(j-q)]-t a(l-q)\} \\
& \quad+\sum_{q=1}^{m} \epsilon_{q}\{s[a(k-q)-a(j-q)]-t[a(l-q)-a(m-q)]\} .
\end{aligned}
$$

By virtue of the independence between $\epsilon_{q}$, it follows that, whenever $k \geq j \geq l \geq m$,

$$
\left|E \exp \left\{\left[i s\left(y_{k, n}^{\prime}-y_{j, n}^{\prime}\right)-i t\left(y_{l, n}^{\prime}-y_{m, n}^{\prime}\right)\right]\right\}\right| \leq I_{1}(s) I_{2}(s, t),
$$

where

$$
\begin{aligned}
I_{1}(s) & =\left|E \exp \left\{i s \sum_{q=j+1}^{k} \epsilon_{q} a(k-q)\right\}\right|, \\
I_{2}(s, t) & =\left|E \exp \left(i \sum_{q=m+1}^{l} \epsilon_{q}\{s[a(k-q)-a(j-q)]-t a(l-q)\}\right)\right| .
\end{aligned}
$$

We may claim that there exist constants $\gamma_{1}>0$ and $\gamma_{2}>0$ such that

$$
\int_{|s| \geq A} I_{1}\left(\frac{s}{\sqrt{n} \phi}\right)\left|\hat{g}\left(-s / c_{n}\right)\right| d s \leq C\left[c_{n} e^{-\gamma_{1} \sqrt{k-j}}+\int_{|s| \geq A} e^{-\gamma_{2}(k-j+1) s^{2} / n} d s\right],
$$

and for each $s \in R$,

$$
\int_{|t| \geq A} I_{2}\left(\frac{s}{\sqrt{n} \phi}, \frac{t}{\sqrt{n} \phi}\right)\left|\hat{g}\left(-t / c_{n}\right)\right| d t \leq C\left[c_{n} e^{-\gamma_{1} \sqrt{l-m}}+\int e^{-\gamma_{2}(l-m+1) t^{2} / n} d t\right](7
$$


In order to prove (7.10), write $\Omega_{1}\left(\Omega_{2}\right.$, respectively) for the set of $m \leq q \leq(l+m) / 2$ such that

$$
|s[a(k-q)-a(j-q)]-t a(l-q)| \geq \sqrt{n}|\phi|
$$

$(|s[a(k-q)-a(j-q)]-t a(l-q)|<\sqrt{n}|\phi|$, respectively). Also let

$$
B_{1}=\sum_{q \in \Omega_{2}} a(l-q)^{2}, \quad B_{2}=\sum_{q \in \Omega_{2}} a(l-q)[a(k-q)-a(j-q)]
$$

and $B_{3}=\sum_{q \in \Omega_{2}}[a(k-q)-a(j-q)]^{2}$. By Hölder's inequality, $B_{2}^{2} \leq B_{3} B_{1}$ and hence

$$
\begin{aligned}
& \sum_{q \in \Omega_{2}}\{s[a(k-q)-a(j-q)]-t a(l-q)\}^{2} \\
= & s^{2} B_{3}-2 s t B_{2}+t^{2} B_{1}=B_{1}\left(t-s B_{2} / B_{1}\right)^{2}+s^{2}\left(B_{3}-B_{2}^{2} / B_{1}\right) \\
\geq & B_{1}\left(t-s B_{2} / B_{1}\right)^{2} .
\end{aligned}
$$

Next, using the fact that there exist constants $\gamma_{1}^{\prime}>0$ and $\gamma_{2}^{\prime}>0$ such that

$$
\left|E e^{i \epsilon_{1} t}\right| \leq \begin{cases}e^{-\gamma_{1}^{\prime}} & \text { if }|t| \geq 1 \\ e^{-\gamma_{2}^{\prime} t^{2}} & \text { if }|t| \leq 1\end{cases}
$$

since $E \epsilon_{1}=0, E \epsilon_{1}^{2}=1$ and $\epsilon_{1}$ satisfies the Cramér condition $\lim \sup _{|t| \rightarrow \infty}\left|E e^{i t \epsilon_{1}}\right|<1$, it follows from the independence of $\epsilon_{t}$ that, for all $l \geq m$,

$$
\begin{aligned}
& I_{2}\left(\frac{s}{\sqrt{n} \phi}, \frac{t}{\sqrt{n} \phi}\right) \\
\leq & \exp \left\{-\gamma_{1}^{\prime} \#\left(\Omega_{1}\right)-\gamma_{2}^{\prime}\left(n \phi^{2}\right)^{-1} \sum_{q \in \Omega_{2}}\{s[a(k-q)-a(j-q)]-t a(l-q)\}^{2}\right\} \\
\leq & \exp \left\{-\gamma_{1}^{\prime} \#\left(\Omega_{1}\right)-\gamma_{2}^{\prime} B_{1}\left(n \phi^{2}\right)^{-1}\left(t-s B_{2} / B_{1}\right)^{2}\right\},
\end{aligned}
$$

where $\#(A)$ denotes the number of elements in $A$. Note that $\Omega_{1}+\Omega_{2}=(l-m) / 2$ and $|a(v)| \geq e^{-|\kappa|}|\phi| / 4$ for all sufficiently large $v$ [see (7.14) in Wang and Phillips (2009b)]. It is readily seen that, there exists a $n_{0}$ such that for all $l-m \geq n_{0}, B_{1} \geq C(l-m)$, whenever $\#\left(\Omega_{1}\right) \leq \sqrt{l-m}$. Using these facts in (7.12), we find that, whenever $l-m \geq n_{0}$,

$$
\begin{aligned}
& \int_{|t| \geq A} I_{2}\left(\frac{s}{\sqrt{n} \phi}, \frac{t}{\sqrt{n} \phi}\right)\left|\hat{g}\left(-t / c_{n}\right)\right| d t \\
\leq & \int_{\#\left(\Omega_{1}\right) \geq \sqrt{l-m}} e^{-\gamma_{1}^{\prime} \#\left(\Omega_{1}\right)}\left|\hat{g}\left(-t / c_{n}\right)\right| d t+C \int_{\#\left(\Omega_{1}\right) \leq \sqrt{l-m}} e^{-\gamma_{2}^{\prime} B_{1}\left(n \phi^{2}\right)^{-1}\left(t-s B_{2} / B_{1}\right)^{2}} d t \\
\leq & \left.C c_{n} e^{-\gamma_{1}^{\prime} \sqrt{l-m}} \int|\hat{g}(t)| d t+C_{1} \int e^{-C \gamma_{2}^{\prime}(l-m) t^{2} / n} d t\right) \\
\leq & C_{2}\left[c_{n} e^{-\gamma_{1} \sqrt{l-m}}+\int e^{-\gamma_{2}(l-m+1) t^{2} / n} d t\right]
\end{aligned}
$$


for some $\gamma_{1}>0$ and $\gamma_{2}>0$. This prove (7.10), since (7.10) is obvious if $0 \leq l-m \leq n_{0}$.

Similarly, it follows from (7.11) and the fact that $|a(v)| \geq e^{-|\kappa|}|\phi| / 4$ for all sufficiently large $v$ that, there exist $\epsilon>0$ and $n_{1}$ such that for all $k-j \geq n_{1}$, if $|s| \geq \epsilon \sqrt{n}$, then

$$
I_{1}\left(\frac{s}{\sqrt{n} \phi}\right) \leq e^{-\gamma_{1}^{\prime}(k-j) / 2}
$$

and if $|s| \leq \epsilon \sqrt{n}$, then

$$
I_{1}\left(\frac{s}{\sqrt{n} \phi}\right) \leq e^{-C \gamma_{2}^{\prime} s^{2} \sum_{q=j+1}^{k} a(k-q)^{2} / n} \leq e^{-\gamma_{1}(k-j+1) s^{2} / n}
$$

for some $\gamma_{2}>0$. By virtue of these facts, it is readily seen that

$$
\begin{aligned}
& \int_{|s| \geq A} I_{1}\left(\frac{s}{\sqrt{n} \phi}\right)\left|\hat{g}\left(-s / c_{n}\right)\right| d s \\
\leq & e^{-\gamma_{1}(k-j) / 2} \int_{|s| \geq \epsilon \sqrt{n}}\left|\hat{g}\left(-s / c_{n}\right)\right| d s+\int_{A \leq|s| \leq \epsilon \sqrt{n}} e^{-\gamma_{2}(k-j+1) s^{2} / n} d s \\
\leq & C\left[c_{n} e^{-\gamma_{1} \sqrt{k-j}}+\int_{|s| \geq A} e^{-\gamma_{2}(k-j+1) s^{2} / n} d s\right] .
\end{aligned}
$$

which yields (7.9).

We are now ready to prove (7.8). Write $\eta_{r}^{\prime \prime}=\sum_{q=-\infty}^{0} \epsilon_{q} \phi_{r-q}$. Note that $\eta_{r}=\eta_{r}^{\prime}+\eta_{r}^{\prime \prime}$. Simple calculations show that

$$
s\left(x_{k, n}-x_{j, n}\right)-t\left(x_{l, n}-x_{m, n}\right)=s\left(y_{k, n}^{\prime}-y_{j, n}^{\prime}\right)-t\left(y_{l, n}^{\prime}-y_{m, n}^{\prime}\right)+F\left(\epsilon_{0}, \epsilon_{-1}, \ldots\right),
$$

where $F\left(\epsilon_{0}, \epsilon_{-1}, \ldots\right)$ depends only on $\epsilon_{j}, j \leq 0$, which is independent of $s\left(y_{k, n}^{\prime}-y_{j, n}^{\prime}\right)-$ $t\left(y_{l, n}^{\prime}-y_{m, n}^{\prime}\right)$. It now follows from (7.9) and (7.10) that

$$
\begin{aligned}
E\left|R_{2 n}(r)\right|^{2} & \leq \frac{C}{n^{4}} \sum_{k, j, l, m=1}^{n} \int_{|s| \geq A} \int_{|t| \geq A}\left|\hat{g}\left(-s / c_{n}\right)\right|\left|\hat{g}\left(-t / c_{n}\right)\right| \\
& \leq \frac{C}{n^{4}} \sum_{k, j, l, m=1}^{n} \int_{|s| \geq A} \int_{|t| \geq A}\left|\hat{g}\left(-s / c_{n}\right)\right|\left|\hat{g}\left(-t / x_{n, n}\right)\right| \\
& \leq \frac{C_{1}}{n^{4}} \sum_{k \geq j \geq l \geq m} \int_{|s| \geq A} I_{1}\left(\frac{s}{\sqrt{n} \phi}\right)\left|\hat{g}\left(-s / c_{n}\right)\right| d s \int_{|t| \geq A} I_{2}\left(\frac{s}{\sqrt{n} \phi}, \frac{t}{\sqrt{n} \phi}\right)\left|\hat{g}\left(-t / c_{n}\right)\right| d t
\end{aligned}
$$




$$
\begin{aligned}
& \leq \frac{C_{2}}{n^{4}} \sum_{1 \leq j \leq k \leq n}\left[c_{n} e^{-\gamma_{1} \sqrt{k-j}}+\int_{|s| \geq A} e^{-\gamma_{2}(k-j+1) s^{2} / n} d s\right] \\
& \times \sum_{1 \leq m \leq l \leq n}\left[c_{n} e^{-\gamma_{1} \sqrt{l-m}}+\int e^{-\gamma_{2}(l-m+1) t^{2} / n} d t\right] \\
& \leq C_{2}\left[\frac{c_{n}}{n^{2}} \sum_{1 \leq j \leq k \leq n} e^{-\gamma_{1} \sqrt{k-j}}+\int_{|s| \geq A} \frac{1}{n^{2}} \sum_{1 \leq j \leq k \leq n} e^{-\gamma_{2}(k-j+1) s^{2} / n} d s\right] \\
& \leq C_{3}\left[\frac{c_{n}}{n}+\int_{|s| \geq A} \int_{0}^{1} \int_{0}^{u} e^{-\gamma_{2}(u-v) s^{2}} d v d u d s\right] \\
& \times\left[\frac{c_{n}}{n}+\iint_{0}^{1} \int_{0}^{u} e^{-\gamma_{1} \sqrt{l-m}}+\int \frac{1}{n^{2}} \sum_{1 \leq m \leq l \leq n} e^{-\gamma_{2}(l-m+1) s^{2} / n} d s\right] \\
& \rightarrow 0,
\end{aligned}
$$

when $n \rightarrow \infty$ first and then $A \rightarrow \infty$. This proves (7.8), and hence the results (5.6) and (5.7) under the additional condition (7.4).

We next remove the additional condition (7.4). Recall $\int_{-\infty}^{\infty}|g(x)| d x<\infty$. We first claim that, for any $\epsilon>0$, we may construct a $g_{\delta_{0}}(x)$ satisfying $(7.4), \int_{-\infty}^{\infty}\left|g_{\delta_{0}}(x)\right| d x<\infty$ and

$$
\int_{-\infty}^{\infty}\left|g(x)-g_{\delta_{0}}(x)\right| d x<\epsilon
$$

Since Theorem 5.1 holds true for $g_{\delta_{0}}(x)$, it remains to show that

$$
\frac{c_{n}}{n^{2}} \sum_{k, j=1}^{n}\left|g\left[c_{n}\left(x_{k, n}-x_{j, n}\right)\right]-g_{\delta_{0}}\left[c_{n}\left(x_{k, n}-x_{j, n}\right)\right]\right|=O_{P}(\epsilon) .
$$

This follows from (7.13) and the assumption that $\int_{-\infty}^{\infty}\left|E e^{i t \epsilon_{0}}\right| d t<\infty$. Indeed, under this assumption, similar arguments to those in the proof of Corollary 3.2 in Wang and Phillips (2009a) show that $\sqrt{n}\left(x_{k, n}-x_{j, n}\right) / \sqrt{k-j}$, for all $1 \leq j<k \leq n$, has a density $h_{k, j}(x)$ which is uniformly bounded by a constant $K$. This fact implies that

$$
\begin{aligned}
& \frac{c_{n}}{n^{2}} \sum_{k, j=1}^{n} E\left|g\left[c_{n}\left(x_{k, n}-x_{j, n}\right)\right]-g_{\delta_{0}}\left[c_{n}\left(x_{k, n}-x_{j, n}\right)\right]\right| \\
= & \frac{2 c_{n}}{n^{2}} \sum_{1 \leq j<k \leq n} \int_{-\infty}^{\infty}\left|g\left(\frac{c_{n} \sqrt{n} x}{\sqrt{k-j}}\right)-g_{\delta_{0}}\left(\frac{c_{n} \sqrt{n} x}{\sqrt{k-j}}\right)\right| h_{k, j}(x) d x+\frac{c_{n}|g(0)|}{n} \\
\leq & 2 K \int_{-\infty}^{\infty}\left|g(x)-g_{\delta_{0}}(x)\right| d x \frac{1}{n^{5 / 2}} \sum_{1 \leq j<k \leq n} \sqrt{k-j}+\frac{c_{n}|g(0)|}{n}=O(\epsilon),
\end{aligned}
$$


which yields (7.14).

It remains only to construct a $g_{\delta_{0}}(x)$ satisfying (7.4), (7.13) and $\int_{-\infty}^{\infty}\left|g_{\delta_{0}}(x)\right| d x<\infty$. This can be done according to the similar idea as in the proof of Theorem 4.2.1 in Borodin and Ibragimov (1995). For the sake of completeness, we describe this process as follows.

First of all, for given $\epsilon>0$, there exists a continuous function $f_{\epsilon}(x)$ having a compact support such that

$$
\int_{-\infty}^{\infty}\left|g(x)-g_{\epsilon}(x)\right| d x \leq \epsilon / 2
$$

See Proposition 15.3.3 of Gasquet and Witomski (1999). Next, set

$$
g_{\delta}(x)=\frac{\delta}{\pi} \int_{-\infty}^{\infty} \frac{\sin ^{2}[(x-y) / \delta]}{(x-y)^{2}} g_{\epsilon}(y) d y
$$

Since

$$
\int_{-\infty}^{\infty} \frac{\sin ^{2}(x)}{x^{2}} e^{i t x} d x= \begin{cases}\pi(1-|t| / 2), & \text { if }|t|<2 \\ 0, & \text { otherwise }\end{cases}
$$

it is readily seen that, for any $\delta>0, g_{\delta}(x)$ is continuous and

$$
\begin{aligned}
\hat{g}_{\delta}(t) & =\int_{-\infty}^{\infty} e^{i t x} g_{\delta}(x) d x \\
& =\frac{1}{\pi} \int_{-\infty}^{\infty} g_{\epsilon}(y) e^{i t y} d y \int_{-\infty}^{\infty} \frac{\sin ^{2}(x)}{x^{2}} e^{i t \delta x} d x
\end{aligned}
$$

has a compact support. That is, $g_{\delta}(x)$ satisfies the condition (7.4). Furthermore, by noting $\int_{-\infty}^{\infty} \frac{\sin ^{2}(x)}{x^{2}} d x=\pi$ and

$$
g_{\delta}(x)=\frac{1}{\pi} \int_{-\infty}^{\infty} \frac{\sin ^{2}(y)}{y^{2}} g_{\epsilon}(x+\delta y) d y
$$

simple calculations show that, as $\delta \rightarrow 0$,

$$
\int_{-\infty}^{\infty}\left|g_{\delta}(x)-g_{\epsilon}(x)\right| d x \leq \frac{1}{\pi} \int_{-\infty}^{\infty} \int_{-\infty}^{\infty} \frac{\sin ^{2}(y)}{y^{2}}\left|g_{\epsilon}(x+\delta y)-g_{\epsilon}(x)\right| d x d y \rightarrow 0 .
$$

Consequently, for any $\epsilon>0$, there exists a $g_{\delta_{0}}(x)$ satisfying $(7.4), \int_{-\infty}^{\infty}\left|g_{\delta_{0}}(x)\right| d x<\infty$ and

$$
\begin{aligned}
\int_{-\infty}^{\infty}\left|g(x)-g_{\delta_{0}}(x)\right| d x & \leq \int_{-\infty}^{\infty}\left|g(x)-g_{\epsilon}(x)\right| d x+\int_{-\infty}^{\infty}\left|g_{\delta_{0}}(x)-g_{\epsilon}(x)\right| d x \\
& \leq \epsilon / 2+\epsilon / 2=\epsilon .
\end{aligned}
$$

This completes the construction of $g_{\delta}(x)$. The proof of Theorem 5.1 is now complete. 
Proof of Theorem 5.2. By checking the proof of Theorem 5.1, the integrability condition $\int_{-\infty}^{\infty}\left|E e^{i t \epsilon_{0}}\right| d t<\infty$, which is stronger than the Cramér condition lim $\sup _{|t| \rightarrow \infty}\left|E e^{i t \epsilon_{0}}\right|<$ 1 , is only used to remove the additional condition (7.4). So, to prove Theorem 5.2, it is sufficient to show that this can be done by an alternative method under the additional condition on $g(x)$. Explicitly, we only need to prove that, for any $\epsilon>0$, there exist $g_{\delta_{0}}^{+}(x)$ and $g_{\delta_{0}}^{-}(x)$ such that $g_{\delta_{0}}^{-}(x) \leq g(x) \leq g_{\delta_{0}}^{+}(x)$, both $g_{\delta_{0}}^{+}(x)$ and $g_{\delta_{0}}^{-}(x)$ satisfy $(7.4)$, $\int_{-\infty}^{\infty}\left(\left|g_{\delta_{0}}^{+}(x)\right|+\left|g_{\delta_{0}}^{-}(x)\right|\right) d x<\infty$ and

$$
\int_{-\infty}^{\infty}\left[g_{\delta_{0}}^{+}(x)-g_{\delta_{0}}^{-}(x)\right] d x<\epsilon
$$

Indeed it follows from these facts that

$$
\begin{aligned}
\frac{c_{n}}{n} \sum_{k, j=1}^{[n r]} g\left[c_{n}\left(x_{k, n}-x_{j, n}\right)\right] & \leq \frac{c_{n}}{n} \sum_{k, j=1}^{[n r]} g_{\delta_{0}}^{+}\left[c_{n}\left(x_{k, n}-x_{j, n}\right)\right] \\
& \rightarrow_{D} \int_{-\infty}^{\infty} g_{\delta_{0}}^{+}(x) d x L_{G}(r, 0) \leq\left(\int_{-\infty}^{\infty} g(x) d x+\epsilon\right) L_{G}(r, 0), \\
\frac{c_{n}}{n} \sum_{k, j=1}^{[n r]} g\left[c_{n}\left(x_{k, n}-x_{j, n}\right)\right] & \geq \frac{c_{n}}{n} \sum_{k, j=1}^{[n r]} g_{\delta_{0}}^{-}\left[c_{n}\left(x_{k, n}-x_{j, n}\right)\right] \\
& \rightarrow \int_{-\infty}^{\infty} g_{\delta_{0}}^{-}(x) d x L_{G}(r, 0) \geq\left(\int_{-\infty}^{\infty} g(x) d x-\epsilon\right) L_{G}(r, 0) .
\end{aligned}
$$

This yields (5.6) since $\epsilon$ is arbitrary. In a similar way we may prove (5.7).

The constructions of $g_{\delta_{0}}^{+}(x)$ and $g_{\delta_{0}}^{-}(x)$ again are similar to those in the proof of Theorem 4.2.1 in Borodin and Ibragimov (1995). To start with, we notice that, for $\forall \epsilon>0$, there exist two continuous functions $g_{\epsilon}^{+}(x)$ and $g_{\epsilon}^{-}(x)$ such that $g_{\epsilon}^{-}(x) \leq g(x) \leq g_{\epsilon}^{+}(x)$ and

$$
\int_{-\infty}^{\infty}\left[g_{\epsilon}^{+}(x)-g_{\epsilon}^{-}(x)\right] d x<\epsilon
$$

See, e.g., part (b) and (c) in the proof of Theorem 4.2.1 in Borodin and Ibragimov (1995). Here $g_{\epsilon}^{ \pm}(x)$ can be chosen such that $\left|g_{\epsilon}^{ \pm}(x)\right| \leq M /\left(1+|x|^{1+b}\right)$ for some $b>0$, under the same conditions on $|g(x)|$. Using $g_{\epsilon}^{+}(x)$ and $g_{\epsilon}^{-}(x)$, as in (7.16), define

$$
\begin{aligned}
& f_{\delta}^{+}(x)=\frac{\delta}{\pi} \int_{-\infty}^{\infty} \frac{\sin ^{2}[(x-y) / \delta]}{(x-y)^{2}} g_{\epsilon}^{+}(y) d y, \\
& f_{\delta}^{-}(x)=\frac{\delta}{\pi} \int_{-\infty}^{\infty} \frac{\sin ^{2}[(x-y) / \delta]}{(x-y)^{2}} g_{\epsilon}^{-}(y) d y .
\end{aligned}
$$

Also write (defining $\sin (y) / y=1$ for $y=0$ )

$$
f(x)=\sum_{n=1}^{\infty} n^{-1-b / 2} \frac{\sin ^{2}(x-n)}{(x-n)^{2}} .
$$


Simple calculation shows that

$$
c_{1}\left(1+|x|^{-1-b / 2}\right) \leq f(x) \leq c_{2}\left(1+|x|^{-1-b / 2}\right)
$$

where $c_{1}>0$ and $c_{2}>0$ are constants. On the other hand, as in the proof of $(7.17)$, for any $\epsilon>0$, there exists a $\delta_{1}$ such that for all $0<\delta \leq \delta_{1}$,

$$
\int_{-\infty}^{\infty}\left|f_{\delta}^{+}(x)-g_{\epsilon}^{+}(x)\right| d x \leq \epsilon, \quad \int_{-\infty}^{\infty}\left|f_{\delta}^{-}(x)-g_{\epsilon}^{-}(x)\right| d x \leq \epsilon .
$$

It can also be proved (see below) that, for any $\epsilon>0$, there exists a $\delta_{2}$ such that for all $0<\delta \leq \delta_{2}$

$$
\left|f_{\delta}^{+}(x)-g_{\epsilon}^{+}(x)\right| d x \leq \epsilon f(x), \quad\left|f_{\delta}^{-}(x)-g_{\epsilon}^{-}(x)\right| d x \leq \epsilon f(x) .
$$

Now the required $g_{\delta_{0}}^{+}(x)$ and $g_{\delta_{0}}^{-}(x)$ can be defined by

$$
g_{\delta_{0}}^{+}(x)=f_{\delta_{0}}^{+}(x)+\epsilon f(x) \quad \text { and } \quad g_{\delta_{0}}^{-}(x)=f_{\delta_{0}}^{-}(x)-\epsilon f(x),
$$

where $\delta_{0}=\min \left\{\delta_{1}, \delta_{2}\right\}$. Indeed, as in the proof of Theorem 5.1, both $g_{\delta_{0}}^{+}(x)$ and $g_{\delta_{0}}^{+}(x)$ satisfy the additional condition (7.4). By virtue of (7.22),

$$
g_{\delta_{0}}^{-}(x) \leq g_{\epsilon}^{-}(x) \leq g(x) \leq g_{\epsilon}^{+}(x) \leq g_{\delta_{0}}^{+}(x)
$$

and by (7.19)-(7.21), we have that $\int_{-\infty}^{\infty}\left(\left|g_{\delta_{0}}^{+}(x)\right|+\left|g_{\delta_{0}}^{-}(x)\right|\right) d x<\infty$ and

$$
\begin{aligned}
\int_{-\infty}^{\infty}\left[g_{\delta_{0}}^{+}(x)-g_{\delta_{0}}^{-}(x)\right] d x \leq & \int_{-\infty}^{\infty}\left[g_{\epsilon}^{+}(x)-g_{\epsilon}^{-}(x)\right] d x+\int_{-\infty}^{\infty}\left|f_{\delta_{0}}^{+}(x)-g_{\epsilon}^{+}(x)\right| d x \\
& +\int_{-\infty}^{\infty}\left|f_{\delta_{0}}^{-}(x)-g_{\epsilon}^{-}(x)\right| d x+2 \epsilon \int_{-\infty}^{\infty} f(x) d x \\
\leq & C \epsilon,
\end{aligned}
$$

where $C$ is a constant.

We next prove (7.22). We have

$$
\begin{aligned}
\left|f_{\delta}^{+}(x)-g_{\epsilon}^{+}(x)\right| & \leq \frac{1}{\pi} \int_{-\infty}^{\infty} \frac{\sin ^{2}(y)}{y^{2}}\left|g_{\epsilon}^{+}(x+\delta y)-g_{\epsilon}^{+}(x)\right| d y \\
& \leq \frac{1}{\pi}\left(\int_{\substack{|x| \leq A \\
y \in R}}+\int_{\substack{|x|>A \\
|\delta y| \geq|x| / 2}}+\int_{\substack{|x|>A|<| x|/ 2\\
| \delta y \mid<}}\right) \frac{\sin ^{2}(y)}{y^{2}}\left|g_{\epsilon}^{+}(x+\delta y)-g_{\epsilon}^{+}(x)\right| d y \\
& :=R_{1 A}+R_{2 A}+R_{3 A},
\end{aligned}
$$

where $A$ is chosen later. Recall that (7.20) and $\left|g_{\epsilon}^{ \pm}(x)\right| \leq M /\left(1+|x|^{1+b}\right)$ for some $b>0$. For any $\epsilon>0$, there exists an $A_{0}>0$ such that, whenever $A \geq A_{0}$,

$$
\begin{aligned}
R_{3 A} & \leq 2 M(A / 2)^{-b / 2}\left[1+(|x| / 2)^{-1-b / 2}\right] I(|x| \geq A) \\
& \leq \epsilon f(x) I(|x| \geq A) / 3
\end{aligned}
$$


For this $A_{0}$, routine calculations yield that there exists a $\delta_{2}$ such that, for all $0<\delta \leq \delta_{2}$,

$$
R_{1 A_{0}} \leq \epsilon f(x) I\left(|x| \leq A_{0}\right) / 3
$$

since $g_{\epsilon}^{+}(x)$ is continuous, and

$$
\begin{aligned}
R_{2 A_{0}} & \leq \frac{M \delta}{\pi} \int_{|y| \geq|x| / 2} y^{-2}\left[|x|^{-1-b}+\left(1+|x+y|^{1+b}\right)^{-1}\right] d y I(|x| \geq A) \\
& \leq \epsilon f(x) I(|x| \geq A) / 3 .
\end{aligned}
$$

Taking $A=A_{0}$ in (7.23), it follows from (7.23)-(7.26) that, for any $\epsilon>0$, there exists a $\delta_{2}$ such that for all $0<\delta \leq \delta_{2},\left|f_{\delta}^{+}(x)-g_{\epsilon}^{+}(x)\right| \leq \epsilon f(x)$. Similarly we have $\left|f_{\delta}^{-}(x)-g_{\epsilon}^{-}(x)\right| \leq$ $\epsilon f(x)$. This proves (7.22) and hence completes the proof of Theorem 5.2.

Proof of Theorem 5.3. The idea for the proof of this theorem is similar to Berkes and Horváth (2006). First notice that, for any $\epsilon>0$, there exists $N_{0}$ such that for all $N \geq N_{0}$,

$$
P\left(\sup _{0 \leq u, v \leq 1}\left|G_{1}(u)-G_{1}(v)\right| \geq N / 2\right) \leq 2 P\left(\sup _{0 \leq u \leq 1}\left|G_{1}(u)\right| \geq N / 4\right) \leq \epsilon .
$$

This, together with (5.3), also implies that, for all $N \geq N_{0}$,

$$
\begin{aligned}
& P\left(\sup _{1 \leq k, j \leq n}\left|x_{k, n}-x_{j, n}\right| \geq N\right) \\
\leq & 2 P\left(\sup _{0 \leq u \leq 1}\left|x_{[n u], n}-G_{1}(u)\right| \geq N / 2\right)+P\left(\sup _{0 \leq u, v \leq 1}\left|G_{1}(u)-G_{1}(v)\right| \geq N / 2\right) \\
\leq & 2 \epsilon .
\end{aligned}
$$

Write

$$
g_{N}(x)= \begin{cases}g(x) & \text { if }|x| \leq N \\ 0 & \text { if }|x|>N\end{cases}
$$

By virtue of the above facts and noting that

$$
\frac{1}{n^{2}} \sum_{k, j=1}^{[n r]} g_{N}\left(x_{k, n}-x_{j, n}\right)=\int_{0}^{r} \int_{0}^{r} g_{N}\left(x_{[n u], n}-x_{[n v], n}\right) d u d v+o_{P}(1),
$$

(5.9) will follow if we prove, for $\forall \epsilon>0$ and $N \geq N_{0}$,

$$
P\left(\Lambda_{N} \geq \epsilon\right) \rightarrow 0, \quad \text { as } n \rightarrow \infty
$$

where

$$
\Lambda_{N}=\int_{0}^{1} \int_{0}^{1}\left|g_{N}\left(x_{[n u], n}-x_{[n v], n}\right)-g_{N}\left[G_{1}(u)-G_{1}(v)\right]\right| d u d v
$$


In fact, for any $\epsilon>0$ and $h>0$, we have

$$
\begin{aligned}
& P\left(\Lambda_{N} \geq \epsilon\right) \leq 2 P\left(\sup _{0 \leq u \leq 1}\left|x_{[n u], n}-G_{1}(u)\right| \geq h / 2\right) \\
& \quad+P\left(\int_{0}^{1} \int_{0}^{1} \sup _{|t| \leq h}\left|g_{N}\left[G_{1}(u)-G_{1}(v)+t\right]-g_{N}\left[G_{1}(u)-G_{1}(v)\right]\right| d u d v \geq \epsilon\right) .
\end{aligned}
$$

By (5.3), we may choose $h=h_{n} \rightarrow 0$ such that

$$
P\left(\sup _{0 \leq u \leq 1}\left|x_{[n u], n}-G_{1}(u)\right| \geq h / 2\right) \rightarrow 0, \quad \text { as } n \rightarrow \infty .
$$

Note that $G_{1}(u)-G_{1}(v) \sim N\left(0, \operatorname{var}\left[G_{1}(u)-G_{1}(v)\right]\right)$. For this chosen $h=h_{n} \rightarrow 0$, it is readily seen that, for some $\alpha>0$,

$$
\begin{aligned}
& P\left(\int_{0}^{1} \int_{0}^{1} \sup _{|t| \leq h}\left|g_{N}\left[G_{1}(u)-G_{1}(v)+t\right]-g_{N}\left[G_{1}(u)-G_{1}(v)\right]\right| d u d v \geq \epsilon\right) \\
\leq & \epsilon^{-1} \int_{0}^{1} \int_{0}^{1} E \sup _{|t| \leq h}\left|g_{N}\left[G_{1}(u)-G_{1}(v)+t\right]-g_{N}\left[G_{1}(u)-G_{1}(v)\right]\right| d u d v \\
\leq & C \epsilon^{-1} \int_{0}^{1} \int_{0}^{1} \frac{1}{\sqrt{|u-v|}} \int_{-\infty|t| \leq h}^{\infty} \sup _{|c|}\left|g_{N}(x+t)-g_{N}(x)\right| e^{-C x^{2} /|u-v|} d x d u d v \\
\leq & C_{1} \epsilon^{-1} \int_{0}^{1} \int_{0}^{1} \frac{1}{|u-v|^{\alpha}} d u d v \int_{-N}^{N}|x|^{\alpha-1} \sup _{|t| \leq h}|g(x+t)-g(x)| d x \\
\leq & C_{2} \epsilon^{-1} \int_{-N}^{N}|x|^{\alpha-1} \sup _{|t| \leq h}|g(x+t)-g(x)| d x \\
\rightarrow & 0, \quad \operatorname{as} n \rightarrow \infty, \quad
\end{aligned}
$$

where we have used (5.8) and the fact that $t^{1-\alpha} e^{-t^{2} / 2} \leq C$ for all $0 \leq t<\infty$. Taking (7.29) and (7.30) into (7.28), we prove (7.27), and also complete the proof of Theorem 3.

\section{Proofs of Propositions 6.1-6.8}

Except where mentioned explicitly, the notation in this section is the same as in previous sections. In Section 8.1, we introduce some preliminary lemmas. The proofs of Propositions 6.1-6.8 will be given in Sections 8.2-8.9 respectively. 
8.1 Preliminaries. First note that

$$
\begin{aligned}
x_{t} & =\sum_{j=1}^{t} \rho^{t-j} \eta_{j}=\sum_{j=1}^{t} \rho^{t-j} \sum_{i=-\infty}^{j} \epsilon_{i} \phi_{j-i} \\
& =\rho^{t-s} x_{s}+\sum_{j=s+1}^{t} \rho^{t-j} \sum_{i=-\infty}^{s} \epsilon_{i} \phi_{j-i}+\sum_{j=s+1}^{t} \rho^{t-j} \sum_{i=s+1}^{j} \epsilon_{i} \phi_{j-i} \\
& :=\rho^{t-s} x_{s}+\Delta_{s, t}+x_{s, t}^{\prime}
\end{aligned}
$$

where

$$
x_{s, t}^{\prime}=\sum_{j=1}^{t-s} \rho^{t-j-s} \sum_{i=1}^{j} \epsilon_{i+s} \phi_{j-i}=\sum_{i=s+1}^{t} \epsilon_{i} \sum_{j=0}^{t-i} \rho^{t-j-i} \phi_{j} .
$$

Write $d_{s, t}^{2}=\sum_{i=s+1}^{t} \rho^{2(t-i)}\left(\sum_{j=0}^{t-i} \rho^{-j} \phi_{j}\right)^{2}=E\left(x_{s, t}^{\prime}\right)^{2}$. Without loss of generality, assume $d_{s, t} \neq 0$ for all $0 \leq s<t \leq n$. Otherwise, $x_{s, t}^{\prime}=0$,a.s. This occurs only in the situation such as $\phi_{0}=\phi_{1}=\ldots=\phi_{k_{0}}=0$ with a finite $k_{0}$. Hence $t-s$ must be small and the main results can be obtained by a routine modification. By virtue of this fact, for all $0 \leq s<t \leq n$, we have that $C_{1}(t-s) \leq d_{s, t}^{2} \leq C_{2}(t-s)$ and

$$
\frac{1}{\sqrt{t-s}} x_{s, t}^{\prime} \text { has a density } h_{s, t}(x),
$$

which is uniformly bounded by a constant $C_{0}$. See (7.14) and Proposition 6.2 (page 1934 there) of Wang and Phillips (2009b) with a minor modification. Furthermore we may prove that,

$$
\text { conditional on } \mathcal{F}_{s}, x_{t} / \sqrt{t} \text { has a density } h_{s, t}\left(x-x_{s}^{*} / \sqrt{t-s}\right) \text {, }
$$

where $x_{s}^{*}=\rho^{t-s} x_{s}+\Delta_{s, t}$, and under Assumption 1,

$$
\begin{aligned}
& \sup _{x}\left|h_{s, t}(x+y)-h_{s, t}(x)\right| \leq C|y| \\
& \left|\Delta_{s, t}\right| \leq e \sum_{i=-\infty}^{s}\left|\epsilon_{i}\right| \sum_{j=s+1}^{t}\left|\phi_{j-i}\right| \leq C \sum_{k=0}^{\infty}(k+1)^{-1-\delta}\left|\epsilon_{s-k}\right| .
\end{aligned}
$$

Indeed (8.3) follows from (8.2) because of the independence between $\mathcal{F}_{s}$ and $\epsilon_{k}, k \geq s+1$ and (8.5) is obvious by recalling $\sum_{k=0}^{\infty} k^{1+\delta}\left|\phi_{k}\right|<\infty$. If we write $\varphi_{s, t}(u)=E e^{i u x_{s, t}^{\prime} / \sqrt{t-s}}$, arguments similar to those in the proof of Corollary 2.2 in Wang and Phillips (2009a) yield that, uniformly for $0 \leq s<t \leq n, \int_{-\infty}^{\infty}(1+|u|)\left|\varphi_{s, t}(u)\right| d u<\infty$. It follows by inversion of the characteristic function $\varphi_{s, t}(u)$ that

$$
\begin{aligned}
\sup _{x}\left|h_{s, t}(x+y)-h_{s, t}(x)\right| & =\frac{1}{\sqrt{2 \pi}}\left|\int_{-\infty}^{\infty}\left(e^{-i u(x+y)}-e^{-i u x}\right) \varphi_{s, t}(u) d u\right| \\
& \leq C|y| \int_{-\infty}^{\infty}|u|\left|\varphi_{s, t}(u)\right| d u \leq C_{1}|y|,
\end{aligned}
$$


which implies (8.4).

By making use of (8.2)-(8.5), we may establish the following lemmas which play a key part in the proofs of our main results.

LEMMA 8.1. Assume that $p(x)$ satisfies $\int|p(x)| d x<\infty$. Let Assumptions 1-2 hold. Then,

(i) for any $0 \leq s<t \leq n$ and $h>0$,

$$
E\left\{p\left(x_{t} / h\right) \mid \mathcal{F}_{s}\right\}=\frac{h}{\sqrt{t-s}} \int_{-\infty}^{\infty} p\left(\rho^{t-s} x_{s} / h+x\right) h_{s, t}\left(\frac{h x}{\sqrt{t-s}}\right) d x+\mathcal{L}_{n}
$$

where

$$
\left|\mathcal{L}_{n}\right| \leq \frac{C h}{t-s}\left(1+\sum_{k=0}^{\infty}(k+1)^{-1-\delta}\left|\epsilon_{s-k}\right|\right) \int_{-\infty}^{\infty}|p(x)| d x
$$

(ii) for any $2 \leq s+2 \leq t \leq n$ and $h>0$,

$$
\left|E\left\{g\left(u_{s+1}\right) p\left(x_{t} / h\right) \mid \mathcal{F}_{s}\right\}\right| \leq \frac{C h}{t-s} \int_{-\infty}^{\infty}|p(x)| d x,
$$

where $g(x)$ satisfies that $E\left[g\left(u_{s+1}\right) \mid \mathcal{F}_{s}\right]=0, s \geq 1$ and $\sup _{s \geq 1} E\left[g\left(u_{s+1}\right)^{2} \mid \mathcal{F}_{s}\right] \leq C$; (iii) for any $0 \leq s<t \leq n$,

$$
E\left(\left|p\left(x_{t} / h\right)\right| \mid \mathcal{F}_{s}\right) \leq \frac{C h}{\sqrt{t-s}} \int_{-\infty}^{\infty}|p(x)| d x
$$

(iv) for any $2 \leq s+2 \leq t \leq n$ and $h>0$,

$$
E\left\{g\left(\left|u_{s+1}\right|\right)\left|p\left(x_{t} / h\right)\right| \mid \mathcal{F}_{s}\right\} \leq \frac{C h E\left(g\left(\left|u_{s+1}\right|\right) \mid \mathcal{F}_{s}\right)}{\sqrt{t-s}} \int_{-\infty}^{\infty}|p(x)| d x ;
$$

(v) for any $s+1 \leq k_{1}, \ldots, k_{m} \leq t$ and $2 \leq s+m+2 \leq t \leq n$ and $h>0$,

$$
E\left\{\left(1+\left|u_{s+1}\right|\right) \prod_{u=1}^{m}\left|\epsilon_{k_{u}}\right|\left|p\left(x_{t} / h\right)\right| \mid \mathcal{F}_{s}\right\} \leq \frac{C h}{\sqrt{t-s-m}} \int_{-\infty}^{\infty}|p(x)| d x .
$$

Proof. By virtue of the independence between $\mathcal{F}_{s}$ and $\epsilon_{k}, k \geq s+1$, it follows from (8.1)-(8.2) that

$$
\begin{aligned}
& E\left\{p\left(x_{t} / h\right) \mid \mathcal{F}_{s}\right\} \\
= & \frac{h}{\sqrt{t-s}} \int_{-\infty}^{\infty} p\left(\rho^{t-s} x_{s} / h+\Delta_{s, t} / h+x\right) h_{s, t}\left(\frac{h x}{\sqrt{t-s}}\right) d x \\
= & \frac{h}{\sqrt{t-s}} \int_{-\infty}^{\infty} p\left(\rho^{t-s} x_{s} / h+x\right) h_{s, t}\left[\frac{h}{\sqrt{t-s}}\left(x-\Delta_{s, t} / h\right)\right] d x \\
= & \frac{h}{\sqrt{t-s}} \int_{-\infty}^{\infty} p\left(\rho^{t-s} x_{s} / h+x\right) h_{s, t}\left(\frac{h x}{\sqrt{t-s}}\right) d x+\mathcal{L}_{n}
\end{aligned}
$$


where, by (8.4)-(8.5),

$$
\begin{aligned}
\mathcal{L}_{n} & \leq \frac{h}{\sqrt{t-s}} \sup _{x}\left|h_{s, t}\left(x-\frac{\Delta_{s, t}}{\sqrt{t-s}}\right)-h_{s, t}(x)\right| \int_{-\infty}^{\infty}\left|p\left(\rho^{t-s} x_{s} / h+x\right)\right| d x \\
& \leq \frac{C h}{t-s}\left|\Delta_{s, t}\right| \int_{-\infty}^{\infty}|p(x)| d x \\
& \leq \frac{C h}{t-s}\left(1+\sum_{k=0}^{\infty}(k+1)^{-1-\delta}\left|\epsilon_{s-k}\right|\right) \int_{-\infty}^{\infty}|p(x)| d x .
\end{aligned}
$$

This proves (8.6). Similarly, it follows from (8.1) and (8.2) that

$$
\begin{aligned}
& E\left\{g\left(u_{s+1}\right) p\left(x_{t} / h\right) \mid \mathcal{F}_{s}\right\} \\
= & \frac{h}{\sqrt{t-s-1}} \int_{-\infty}^{\infty} E\left\{g\left(u_{s+1}\right) p\left(\frac{\rho^{t-s} x_{s}}{h}+\frac{\Delta_{s, t}}{h}+\frac{\left(x_{s, t}^{\prime}-x_{s+1, t}^{\prime}\right)}{h}+y\right) \mid \mathcal{F}_{s}\right\} \\
= & \frac{h}{\sqrt{t-s-1}} \int_{-\infty}^{\infty} p\left(\frac{\rho_{s+1, t}^{t-s} x_{s}}{h}+\frac{\Delta_{s, t}}{h}+y\right) d y \\
& E\left\{g\left(u_{s+1}\right) h_{s+1, t}\left[\frac{h}{\sqrt{t-s-1}}\left\{y-\left(x_{s, t}^{\prime}-x_{s+1, t}^{\prime}\right) / h\right\}\right] \mid \mathcal{F}_{s}\right\} d y .
\end{aligned}
$$

This, together with $E\left(g\left(u_{s+1}\right) \mid \mathcal{F}_{s}\right)=0$ and (8.4), yields

$$
\begin{aligned}
& \left|E\left\{u_{s+1} p\left(x_{t} / h\right) \mid \mathcal{F}_{s}\right\}\right| \\
\leq & \frac{C h}{t-s} \int_{-\infty}^{\infty}\left|p\left(\frac{\rho^{t-s} x_{s}}{h}+\frac{\Delta_{s, t}}{h}+y\right)\right| d y E\left\{\left|g\left(u_{s+1}\right)\right|\left|x_{s, t}^{\prime}-x_{s+1, t}^{\prime}\right| \mid \mathcal{F}_{s}\right\} \\
\leq & \frac{C h}{t-s} \int_{-\infty}^{\infty}|p(y)| d y,
\end{aligned}
$$

where we have used the fact:

$$
E\left\{\left|g\left(u_{s+1}\right)\right|\left|x_{s, t}^{\prime}-x_{s+1, t}^{\prime}\right| \mid \mathcal{F}_{s}\right\} \leq C\left(E\left\{g^{2}\left(u_{s+1}\right) \mid \mathcal{F}_{s}\right\}\right)^{1 / 2}\left(E \epsilon_{1}^{2}\right)^{1 / 2} \leq C .
$$

This proves (8.7). The proofs of (8.8) and (8.9) are simple. Note that, whenever $2 \leq$ $s+m+2 \leq t \leq n$, similar to (8.2) we find that

$$
\frac{1}{\sqrt{t-s-m}}\left(x_{s+1, t}^{\prime}-\sum_{u=1}^{m} \epsilon_{k_{u}} \sum_{j=0}^{t-k_{u}} \rho^{t-k_{u}-j} \phi_{j}\right) \text { has a density, say } h_{s, t}^{\prime}(x)
$$

which is uniformly bounded by a constant $C_{0}$. It follows from (8.2) and independence 
between $\epsilon_{j}$ that, for $k_{1}, \ldots, k_{m} \leq t$ and $2 \leq s+m+2 \leq t \leq n$,

$$
\begin{aligned}
& E\left\{\left(1+\left|u_{s+1}\right|\right) \prod_{u=1}^{m}\left|\epsilon_{k_{u}}\right|\left|p\left(x_{t} / h\right)\right| \mid \mathcal{F}_{s}\right\} \\
= & \frac{h}{\sqrt{t-s}} E\left\{\left(1+\left|u_{s+1}\right|\right) \prod_{u=1}^{m}\left|\epsilon_{k_{u}}\right|\right. \\
& \left.\int_{-\infty}^{\infty}\left|p\left(\frac{\rho^{t-s-1} x_{s+1}}{h}+\frac{\Delta_{s+1, t}}{h}+\frac{\sum_{u=1}^{m} \epsilon_{k_{u}} \sum_{j=0}^{t-k_{u}} \rho^{t-k_{u}-j} \phi_{j}}{h}+y\right)\right|\left|h_{s, t}^{\prime}\left(\frac{y h}{\sqrt{t-s}}\right)\right| d y \mid \mathcal{F}_{s}\right\} \\
\leq & \frac{C_{0} h}{\sqrt{t-s}} \int_{-\infty}^{\infty}|p(y)| d y E\left[\left(1+\left|u_{s+1}\right|\right) \prod_{u=1}^{m}\left|\epsilon_{k_{u}}\right| \mid \mathcal{F}_{s}\right] \\
\leq & \frac{C h}{\sqrt{t-s}} \int_{-\infty}^{\infty}|p(y)| d y,
\end{aligned}
$$

which yields (8.10). The proof of Lemma 8.1 is now complete.

LEMMA 8.2. Let $p_{j}(x), j=1,2,3$, be positive functions satisfying $\int_{-\infty}^{\infty} p_{j}(x) d x<\infty$ and $\sup _{x} p_{j}(x)<\infty$. Then, under Assumption 1, we have

$$
\begin{aligned}
& E p_{1}\left[\left(x_{t}-x_{i}\right) / h\right] \leq \frac{C h}{\sqrt{t-i}} \int_{-\infty}^{\infty} p_{1}(x) d x \\
& E p_{1}\left[\left(x_{t}-x_{i}\right) / h\right] p_{2}\left[\left(x_{t}-x_{j}\right) / h\right] \\
& \quad \leq \frac{C h^{2}}{\sqrt{t-j} \sqrt{j-i}} \int_{-\infty}^{\infty} p_{1}(x) d x \int_{-\infty}^{\infty} p_{2}(y) d y, \quad \text { for } i<j \\
& E p_{1}\left[\left(x_{t}-x_{i}\right) / h\right] p_{2}\left[\left(x_{t}-x_{j}\right) / h\right] p_{3}\left[\left(x_{t}-x_{k}\right) / h\right] \\
& \quad \leq \frac{C h^{3}}{\sqrt{t-k} \sqrt{k-j} \sqrt{j-i}} \int_{-\infty}^{\infty} p_{1}(y) d y \int_{-\infty}^{\infty} p_{2}(y) d y \int_{-\infty}^{\infty} p_{3}(y) d y,
\end{aligned}
$$

for $i<j<k$. For the $K(x)$ defined in Assumption 3, write

$$
\chi_{s, t}(i, j, k, l)=E\left\{K\left[\left(x_{s}-x_{i}\right) / h\right] K\left[\left(x_{s}-x_{j}\right) / h\right] K\left[\left(x_{t}-x_{k}\right) / h\right] K\left[\left(x_{t}-x_{l}\right) / h\right]\right\} .
$$

Similar to (8.11)-(8.13), we also have

(i) if $t>s, s-2 \geq i, j, i \neq j, k=i$ and $l=j$ or $l=s-1$ or $l=s$ then

$$
\chi_{s, t}(i, j, k, l) \leq \frac{C h^{3}}{\sqrt{t-s}} \frac{1}{\sqrt{s-i_{1}}},
$$

where $i_{1}=\max \{i, j\}$;

(ii) if $t>s, s-2 \geq i, j, k, i \neq j \neq k$ and $l=i$ or $l=s-1$ or $l=s$, then

$$
\chi_{s, t}(i, j, k, l) \leq \frac{C h^{4}}{\sqrt{t-s}} \frac{1}{\sqrt{s-i_{1}}} \frac{1}{\sqrt{i_{2}-i_{3}}},
$$

where $i_{1}=\max \{i, j, k\}, i_{3}=\min \{i, j, k\}$ and $i_{2}$ is the median value of $i, j, k$. 
Proof. We only prove (8.13) and (8.15) for $i<j<k$ and $l=s-1$. The other results are similar but simpler. In order to prove (8.13), let $p^{*}(x)=p_{1}\left(x-x_{i} / h\right) p_{2}(x-$ $\left.x_{j} / h\right) p_{3}\left(x-x_{k} / h\right)$. Note that $p^{*}(x)$ is $\mathcal{F}_{k}$-measurable and

$$
\begin{aligned}
\int_{-\infty}^{\infty} p^{*}(x) d x & =\int_{-\infty}^{\infty} p_{1}\left[x+\left(x_{k}-x_{i}\right) / h\right] p_{2}\left[x+\left(x_{k}-x_{j}\right) / h\right] p_{3}(x) d x \\
& \leq C \int_{-\infty}^{\infty} p_{3}(x) d x<\infty .
\end{aligned}
$$

It follows from (8.8) with $p(x)=p^{*}(x)$ that

$$
\begin{aligned}
E p^{*}(x) & =E\left\{E\left[p^{*}(x) \mid \mathcal{F}_{k}\right]\right\} \\
& \leq \frac{c h}{\sqrt{t-s}} \int_{-\infty}^{\infty} E\left\{p_{1}\left[x+\left(x_{k}-x_{i} / h\right)\right] p_{2}\left[x+\left(x_{k}-x_{j}\right) / h\right]\right\} p_{3}(x) d x \\
& \leq \frac{C h^{3}}{\sqrt{t-k} \sqrt{k-j} \sqrt{j-i}} \int_{-\infty}^{\infty} p_{1}(y) d y \int_{-\infty}^{\infty} p_{2}(y) d y \int_{-\infty}^{\infty} p_{3}(y) d y,
\end{aligned}
$$

where, in the last inequality, we have used the result (8.12). This proves (8.13).

The idea to prove (8.15) for $i<j<k$ and $l=s-1$ is similar. Indeed, by using (8.8), we have

$$
\begin{aligned}
M_{s} & :=E\left\{K\left[\left(x_{t}-x_{k}\right) / h\right] K\left[\left(x_{t}-x_{s-1}\right) / h\right] \mid \mathcal{F}_{s}\right\} \\
& \leq \frac{C h}{\sqrt{t-s}} \int_{-\infty}^{\infty} E\left\{K\left[x+\left(x_{s-1}-x_{k} / h\right)\right]\right\} K(x) d x, \\
N_{s} & :=E\left\{K\left[\left(x_{s}-x_{i}\right) / h\right] K\left[\left(x_{s}-x_{j}\right) / h\right] \mid \mathcal{F}_{s-1}\right\} \\
& \leq C h \int_{-\infty}^{\infty} E\left\{K\left[x+\left(x_{j}-x_{i}\right) / h\right]\right\} K(x) d x,
\end{aligned}
$$

Similarly, uniformly on $x, y \in R$, it follows from (8.8) that

$$
\begin{aligned}
E\{K & {\left.\left[x+\left(x_{j}-x_{i}\right) / h\right] K\left[y+\left(x_{s-1}-x_{k}\right) / h\right]\right\} } \\
& =E\left\{K\left[x+\left(x_{j}-x_{i}\right) / h\right] E\left(K\left[y+\left(x_{s-1}-x_{k}\right) / h\right] \mid \mathcal{F}_{k}\right)\right\} \\
& \leq \frac{C h}{\sqrt{s-k}} E\left\{K\left[x+\left(x_{j}-x_{i}\right) / h\right] \int_{-\infty}^{\infty} K\left(y+z-x_{k} / h\right) d x\right\} \\
& \leq \frac{C_{1} h}{\sqrt{s-k}} E K\left[x+\left(x_{j}-x_{i}\right) / h\right] \\
& \leq \frac{C h^{2}}{\sqrt{s-k}} \frac{1}{\sqrt{j-i}} .
\end{aligned}
$$


These facts, together with conditional arguments, yield that

$$
\begin{aligned}
& \chi_{s, t}(i, j, k, s-1)=E\left\{K\left[\left(x_{s}-x_{i}\right) / h\right] K\left[\left(x_{s}-x_{j}\right) / h\right] M_{s}\right\} \\
\leq & \frac{C h}{\sqrt{t-s}} \int_{-\infty}^{\infty} E\left\{K\left[\left(x_{s}-x_{i}\right) / h\right] K\left[\left(x_{s}-x_{j}\right) / h\right] K\left[x+\left(x_{s-1}-x_{k} / h\right)\right]\right\} K(x) d x \\
= & \frac{C h}{\sqrt{t-s}} \int_{-\infty}^{\infty} E\left\{K\left[x+\left(x_{s-1}-x_{k} / h\right)\right] N_{s}\right\} K(x) d x \\
\leq & \frac{C h^{2}}{\sqrt{t-s}} \int_{-\infty}^{\infty} \int_{-\infty}^{\infty} E\left\{K\left[x+\left(x_{j}-x_{i}\right) / h\right] K\left[y+\left(x_{s-1}-x_{k}\right) / h\right]\right\} K(x) K(y) d x d y \\
\leq & \frac{C h^{4}}{\sqrt{t-s}} \frac{1}{\sqrt{s-k}} \frac{1}{\sqrt{j-i}}
\end{aligned}
$$

This proves (8.13) for $i<j<k$ and $l=s-1$. The proof of Lemma 8.2 is now complete.

LEMMA 8.3. Write

$$
\begin{array}{r}
I(i, j, k, l, s, t)=E\left\{u_{i+1} u_{j+1} u_{k+1} u_{l+1} K\left[\left(x_{s}-x_{i}\right) / h\right] K\left[\left(x_{s}-x_{j}\right) / h\right]\right. \\
\left.K\left[\left(x_{t}-x_{k}\right) / h\right] K\left[\left(x_{t}-x_{l}\right) / h\right]\right\} .
\end{array}
$$

In addition to Assumptions 1-3, assume $\left|u_{j}\right| \leq A$. Then,

(i) for $t>s, s-2 \geq i, j, k, l$ and $i<j<k<l$,

$$
|I(i, j, k, l, s, t)| \leq C h^{4}\left(\frac{1}{t-s} \frac{1}{\sqrt{s-l}}+\frac{1}{\sqrt{t-s}} \frac{1}{s-l}\right) \frac{1}{\sqrt{l-k}} \frac{1}{\sqrt{j-i}} ;
$$

(ii) for $t \geq l+2,1 \leq i<j \leq s-2, l \geq s+1$ and $k<l$

$$
|I(i, j, k, l, s, t)| \leq \begin{cases}\frac{C h^{4}}{t-l} \frac{1}{\sqrt{l-k}} \frac{1}{\sqrt{s-j}} \frac{1}{\sqrt{j-i}}, & \text { if } k \geq s \\ \frac{C h^{4}}{t-l} \frac{1}{\sqrt{l-s}} \frac{1}{\sqrt{s-j}} \frac{1}{\sqrt{j-i}}, & \text { if } k<s .\end{cases}
$$

(iii) for $t=s$ and $s-2 \geq i, j, k, l$ and $i<j<k<l$,

$$
|I(i, j, k, l, t, t)| \leq \frac{C_{1} h^{4}}{t-l} \frac{1}{\sqrt{l-k}} \frac{1}{\sqrt{k-j}} \frac{1}{\sqrt{j-i}} .
$$

Proof. First for (8.17). It follows (8.6) of Lemma 8.1 with $p(x)=K\left(x-x_{k} / h\right) K(x-$ $\left.x_{l} / h\right)$ that

$$
\begin{aligned}
T_{s} & :=E\left\{K\left[\left(x_{t}-x_{k}\right) / h\right] K\left[\left(x_{t}-x_{l}\right) / h\right] \mid \mathcal{F}_{s}\right\} \\
& =\frac{h}{\sqrt{t-s}} \int_{-\infty}^{\infty} p\left(\rho^{t-s} x_{s} / h+x\right) h_{s, t}\left(\frac{h x}{\sqrt{t-s}}\right) d x+\mathcal{L}_{n}
\end{aligned}
$$


where

$$
\left|\mathcal{L}_{n}\right| \leq \frac{C h}{t-s}\left(1+\sum_{u=0}^{\infty}(u+1)^{-1-\delta}\left|\epsilon_{s-u}\right|\right) \int_{-\infty}^{\infty}|p(x)| d x
$$

Now conditional arguments, together with $\left|h_{s t}(y)\right| \leq C_{0}$ and some simple calculations, yield that

$$
\begin{aligned}
& |I(i, j, k, l, s, t)| \\
= & \left|E\left\{u_{i+1} u_{j+1} u_{k+1} u_{l+1} K\left[\left(x_{s}-x_{i}\right) / h\right] K\left[\left(x_{s}-x_{j}\right) / h\right] T_{s}\right\}\right| \\
\leq & \frac{C_{0} h}{\sqrt{t-s}} \int_{-\infty}^{\infty} \mid E\left\{u_{i+1} u_{j+1} u_{k+1} u_{l+1} K\left[\left(x_{s}-x_{i}\right) / h\right] K\left[\left(x_{s}-x_{j}\right) / h\right]\right. \\
& \left.p\left(\rho^{t-s} x_{s} / h+x\right)\right\} \mid d x+A^{4} E\left\{K\left[\left(x_{s}-x_{i}\right) / h\right] K\left[\left(x_{s}-x_{j}\right) / h\right]\left|\mathcal{L}_{n}\right|\right\} \\
\leq & \frac{C_{0} h}{\sqrt{t-s}} \int_{-\infty}^{\infty}\left|E I_{1}(x)\right| K(x) d x+\frac{C h}{t-s}\left(\int_{-\infty}^{\infty} E\left|I_{2}(x)\right| K(x) d x\right. \\
+ & \left.\sum_{u=0}^{\infty}(u+1)^{-1-\delta} \int_{-\infty}^{\infty} E\left\{\left|\epsilon_{s-u}\right|\left|I_{2}(x)\right|\right\} K(x) d x\right),
\end{aligned}
$$

where

$$
\begin{aligned}
I_{1}(x) & =u_{i+1} u_{j+1} u_{k+1} u_{l+1} K\left[\left(x_{s}-x_{i}\right) / h\right] K\left[\left(x_{s}-x_{j}\right) / h\right] K\left[x+\left(x_{l}-x_{k}\right) / h\right] \\
I_{2}(x) & =K\left[\left(x_{s}-x_{i}\right) / h\right] K\left[\left(x_{s}-x_{j}\right) / h\right] K\left[x+\left(x_{l}-x_{k}\right) / h\right] .
\end{aligned}
$$

It follows from $(8.7)$ with $p(y)=K\left(y-x_{i} / h\right) K\left(y-x_{j} / h\right)$ that

$$
\begin{aligned}
T_{1 s} & :=\left|E\left\{u_{l+1} K\left[\left(x_{s}-x_{i}\right) / h\right] K\left[\left(x_{s}-x_{j}\right) / h\right] \mid \mathcal{F}_{l}\right\}\right| \\
& \leq \frac{C h}{s-l} \int_{-\infty}^{\infty} K\left(y-x_{i} / h\right) K\left(y-x_{j} / h\right) d y \\
& =\frac{C h}{s-l} \int_{-\infty}^{\infty} K(y) K\left[y+\left(x_{j}-x_{i}\right) / h\right] d y .
\end{aligned}
$$

Hence, using conditional arguments and (8.8) repeatedly, we have

$$
\begin{aligned}
\left|E I_{1}(x)\right| & \leq A^{3} E\left(K\left[x+\left(x_{l}-x_{k}\right) / h\right]\left|T_{1 s}\right|\right) \\
& \leq \frac{C h}{s-l} \int_{-\infty}^{\infty} E\left\{K\left[x+\left(x_{l}-x_{k}\right) / h\right] K\left[y+\left(x_{j}-x_{i}\right) / h\right]\right\} K(y) d y \\
& \leq \frac{C_{1} h}{s-l} \frac{h}{\sqrt{l-k}} \frac{h}{\sqrt{j-l}} \int_{-\infty}^{\infty} \int_{-\infty}^{\infty} \int_{-\infty}^{\infty} K(x+z) K(y+u) K(y) d y d z d u \\
& \leq \frac{C_{2} h^{3}}{s-l} \frac{1}{\sqrt{l-k}} \frac{1}{\sqrt{j-i}} .
\end{aligned}
$$


The same idea as in the proof of (8.21), but with (8.10) instead of (8.8), yields that

$$
E\left|I_{2}(x)\right|+E\left\{\left|\epsilon_{s-u}\right|\left|I_{2}(x)\right|\right\} \leq \frac{C h^{3}}{\sqrt{s-l}} \frac{1}{\sqrt{l-k}} \frac{1}{\sqrt{j-i}},
$$

for all $0 \leq u<\infty$. Note that $\sum_{u=0}^{\infty}(u+1)^{-1-\delta}<\infty$. Taking (8.21) and (8.22) into (8.20), we obtain

$$
|I(i, j, k, l, s, t)| \leq C h^{4}\left(\frac{1}{t-s} \frac{1}{\sqrt{s-l}}+\frac{1}{\sqrt{t-s}} \frac{1}{s-l}\right) \frac{1}{\sqrt{l-k}} \frac{1}{\sqrt{j-i}},
$$

which yields (8.17).

Next for (8.19). It follows from (8.7) with $p(y)=K\left(y-x_{i} / h\right) K\left(y-x_{j} / h\right) K(y-$ $\left.x_{k} / h\right) K\left(y-x_{l} / h\right)$ that

$$
\begin{aligned}
& \left|E\left\{u_{l+1} K\left[\left(x_{s}-x_{i}\right) / h\right] K\left[\left(x_{s}-x_{j}\right) / h\right] K\left[\left(x_{s}-x_{k}\right) / h\right] K\left[\left(x_{s}-x_{l}\right) / h\right] \mid \mathcal{F}_{l}\right\}\right| \\
& \leq \frac{C h}{s-l} \int_{-\infty}^{\infty} K\left(y-x_{i} / h\right) K\left(y-x_{j} / h\right) K\left(y-x_{k} / h\right) K\left(y-x_{l} / h\right) d y \\
& \quad=\frac{C h}{s-l} \int_{-\infty}^{\infty} K\left[y+\left(x_{l}-x_{i}\right) / h\right] K\left[y+\left(x_{l}-x_{j}\right) / h\right] K\left[y+\left(x_{l}-x_{k}\right) / h\right] K(y) d y .
\end{aligned}
$$

This, together with (8.13) in Lemma 8.2, yields that

$$
\begin{aligned}
& |I(i, j, k, l, s, s)| \\
\leq & \frac{C A^{3} h}{s-l} \int_{-\infty}^{\infty} E\left\{K\left[y+\left(x_{l}-x_{i}\right) / h\right] K\left[y+\left(x_{l}-x_{j}\right) / h\right] K\left[y+\left(x_{l}-x_{k}\right) / h\right]\right\} K(y) d y \\
\leq & \frac{C_{1} h^{4}}{s-l} \frac{1}{\sqrt{l-k}} \frac{1}{\sqrt{k-j}} \frac{1}{\sqrt{j-i}}\left(\int_{-\infty}^{\infty} K(y) d y\right)^{4} \\
\leq & \frac{C_{1} h^{4}}{s-l} \frac{1}{\sqrt{l-k}} \frac{1}{\sqrt{k-j}} \frac{1}{\sqrt{j-i}},
\end{aligned}
$$

which implies (8.19).

Finally for(8.18). The same idea as above, together with (8.13) in Lemma 8.2, yields that

$$
\begin{aligned}
& |I(i, j, k, l, s, t)| \\
\leq & A^{3} E\left(K\left[\left(x_{s}-x_{i}\right) / h\right] K\left[\left(x_{s}-x_{j}\right) / h\right]\right. \\
& \left.\left|E\left\{u_{l+1} K\left[\left(x_{t}-x_{k}\right) / h\right] K\left[\left(x_{t}-x_{l}\right) / h\right] \mid \mathcal{F}_{l}\right\}\right|\right) \\
\leq & \frac{C h}{t-l} \int_{-\infty}^{\infty} E\left\{K\left[\left(x_{s}-x_{i}\right) / h\right] K\left[\left(x_{s}-x_{j}\right) / h\right] K\left[x+\left(x_{l}-x_{k}\right) / h\right]\right\} K(x) d x \\
\leq & \begin{cases}\frac{C h^{2}}{t-l} \frac{1}{\sqrt{l-k}} E\left\{K\left[\left(x_{s}-x_{i}\right) / h\right] K\left[\left(x_{s}-x_{j}\right) / h\right]\right\}, & \text { if } k \geq s, \\
\frac{C h^{2}}{t-l} \frac{1}{\sqrt{l-s}} E\left\{K\left[\left(x_{s}-x_{i}\right) / h\right] K\left[\left(x_{s}-x_{j}\right) / h\right]\right\}, & \text { if } k<s,\end{cases} \\
\leq & \begin{cases}\frac{C h^{4}}{t-l} \frac{1}{\sqrt{l-k}} \frac{1}{\sqrt{s-j}} \frac{1}{\sqrt{j-i}}, & \text { if } k \geq s, \\
\frac{C h^{4}}{t-l} \frac{1}{\sqrt{l-s}} \frac{1}{\sqrt{s-j}} \frac{1}{\sqrt{j-i}}, & \text { if } k<s .\end{cases}
\end{aligned}
$$


The proof of Lemma 8.3 is complete.

LEMMA 8.4. Suppose Assumption 1 holds. For any $1 \leq s<t$ and $\alpha>0$, we have

$$
E\left(\left|x_{t}\right|^{\alpha-1}\right) \leq C t^{(\alpha-1) / 2}
$$

provided $E\left|\epsilon_{0}\right|^{\max \{[\alpha], 2\}}<\infty$;

$$
E\left\{\left|x_{t}\right|^{\alpha} \mid \mathcal{F}_{s}\right\} \leq C\left[(t-s)^{\alpha / 2}+\left|x_{s}\right|^{\alpha}+\left(\sum_{k=0}^{\infty}(k+1)^{-1-\delta}\left|\epsilon_{s-k}\right|\right)^{\alpha}\right],
$$

provided $E\left|\epsilon_{0}\right|^{\max \{[\alpha]+1,2\}}<\infty$.

Proof. If $\alpha-1$ is an integer greater than 2, (8.23) is well-known. If $\alpha \geq 1$, it follows from $\left|x_{t}\right|^{\alpha-1} \leq t^{(\alpha-1) / 2}\left(1+\left|x_{t} / \sqrt{t}\right|^{\max \{[\alpha], 2\}}\right)$ that

$$
E\left(\left|x_{t}\right|^{\alpha-1}\right) \leq t^{(\alpha-1) / 2}\left(1+E\left|x_{t} / \sqrt{t}\right|^{\max \{[\alpha], 2\}}\right) \leq C t^{(\alpha-1) / 2} .
$$

If $1 \geq \alpha>0$, by recalling (8.3), we have

$$
\begin{aligned}
E\left(\left|x_{t} / \sqrt{t}\right|^{\alpha-1} \mid \mathcal{F}_{0}\right) & =\int_{-\infty}^{\infty}|x|^{\alpha-1} p_{0, t}\left(x-x_{0}^{*} / \sqrt{t}\right) d x \\
& \leq C_{0} \int_{|x| \leq 1}|x|^{\alpha-1} d x+\int_{|x| \geq 1} p_{0, t}\left(x-x_{0}^{*} / \sqrt{t}\right) d x \leq C
\end{aligned}
$$

and hence $E\left(\left|x_{t}\right|^{\alpha-1}\right) \leq C t^{(\alpha-1) / 2}$. Combining all these fact, we obtain (8.23).

Recall $|\rho| \leq C$ and $\sum_{j=1}^{\infty}\left|\phi_{j}\right|<\infty$. It is readily seen that $E\left|x_{s, t}^{\prime}\right|^{\alpha} \leq C(t-s)^{\alpha / 2}$, where $x_{s, t}^{\prime}$ is defined as in (8.1). Now the result (8.24) follows from (8.1), (8.2) and the fact that, whenever $\alpha \geq 0$,

$$
(|x|+|y|+|z|)^{\alpha} \leq C_{\alpha}\left(|x|^{\alpha}+|y|^{\alpha}+|z|^{\alpha}\right)
$$

where $C_{\alpha}$ is a constant depending only on $\alpha$.

LEMMA 8.5. Suppose that Assumptions 1-2 hold. E $\left|\epsilon_{0}\right|^{\max \{[\alpha], 2\}}<\infty$ and $p(x)$ satisfies $\int\left(|p(x)|+p^{2}(x)\right) d x<\infty$. Then, for any $\alpha>0, h>0$ and $1 \leq s \leq n-1$,

$$
E\left\{\left|u_{s+1}\right|\left(1+\left|x_{s}\right|^{\alpha-1}\right)\left|p\left[\left(x_{s+1}-x_{s}\right) / h\right]\right|\right\} \leq C h^{1 / 2}\left(1+s^{(\alpha-1) / 2}\right)
$$

for any $\alpha>0, h>0$ and $t \geq s+2$,

$$
E\left\{\left(1+\left|u_{s+1}\right|\right)\left(1+\left|x_{s}\right|^{\alpha-1}\right)\left|p\left[\left(x_{t}-x_{s}\right) / h\right]\right|\right\} \leq C h\left(1+s^{(\alpha-1) / 2}\right) / \sqrt{t-s} .
$$


If in addition $\int|x|^{\max \left\{\left[\alpha_{1}\right],\left[\alpha_{2}\right]\right\}+1}|p(x)| d x<\infty$ and $E\left|\epsilon_{0}\right|^{\left[\alpha_{1}\right]+\left[\alpha_{2}\right]+2}<\infty$, then for any $\alpha_{1}, \alpha_{2} \geq 0, h>0$ and $0 \leq s<t<i$,

$$
E\left\{\left(1+\left|x_{s}\right|^{\alpha_{1}}\right)\left(1+\left|x_{t}\right|^{\alpha_{2}}\right)\left|p\left[\left(x_{i}-x_{t}\right) / h\right]\right|\left|p\left[\left(x_{i}-x_{s}\right) / h\right]\right|\right\} \leq \frac{C h^{2} s^{\alpha_{1} / 2} t^{\alpha_{2} / 2}}{\sqrt{t-s} \sqrt{i-t}}
$$

for any $\alpha_{1}, \alpha_{2} \geq 0, h>0$ and $t \neq s, s+1$,

$$
\begin{aligned}
E\left\{g\left(u_{s+1}\right)\right. & \left.g_{1}\left(u_{t+1}\right)\left(1+\left|x_{s}\right|^{\alpha_{1}}\right)\left(1+\left|x_{t}\right|^{\alpha_{2}}\right) K\left[\left(x_{t}-x_{s}\right) / h\right]\right\} \\
\leq & \frac{C h s^{\alpha_{1} / 2} t^{\alpha_{2} / 2}}{\sqrt{t-s}}
\end{aligned}
$$

where $g(x)$ and $g_{1}(x)$ are positive real functions such that

$$
\sup _{s \geq 1} E\left\{\left[\left|g\left(u_{s+1}\right)\right|+\left|g_{1}\left(u_{s+1}\right)\right|\right] \mid \mathcal{F}_{s}\right\}<\infty .
$$

Proof. We only prove (8.27). By using conditional arguments and Lemmas 8.1-8.4, the other results are similar but simpler. Note that

$$
\begin{aligned}
& 1+\left|x_{t}\right|^{\alpha_{2}} \leq t^{\alpha_{2} / 2}\left[1+\left(x_{t} / \sqrt{t}\right)^{\alpha_{2}}\right] \\
\leq & C_{\alpha} t^{\alpha_{2} / 2}\left[1+\left(\left|x_{t}-x_{i}\right| / \sqrt{t}\right)^{\left[\alpha_{2}\right]+1}+\left(\left|x_{s}-x_{i}\right| / \sqrt{t}\right)^{\left[\alpha_{2}\right]+1}+\left(\left|x_{s}\right| / \sqrt{t}\right)^{\left[\alpha_{2}\right]+1}\right] .
\end{aligned}
$$

We have that

$$
\begin{aligned}
& E\left\{\left(1+\left|x_{s}\right|^{\alpha_{1}}\right)\left(1+\left|x_{t}\right|^{\alpha_{2}}\right)\left|p\left[\left(x_{i}-x_{t}\right) / h\right]\right|\left|p\left[\left(x_{i}-x_{s}\right) / h\right]\right|\right\} \\
\leq & C_{\alpha} t^{\alpha_{2} / 2} E\left\{\left(1+\left|x_{s}\right|^{\alpha_{1}}\right)\left[1+\left(\left|x_{s}\right| / \sqrt{t}\right)^{\left[\alpha_{2}\right]+1}\right]\left|p\left[\left(x_{i}-x_{t}\right) / h\right]\right|\left|p\left[\left(x_{i}-x_{s}\right) / h\right]\right|\right\} \\
& +C_{\alpha} t^{\alpha_{2} / 2} E\left\{\left(1+\left|x_{s}\right|^{\alpha_{1}}\right) p_{\left[\alpha_{2}\right]+1}\left[\left(x_{i}-x_{t}\right) / h\right]|| p\left[\left(x_{i}-x_{s}\right) / h\right] \mid\right\} \\
& +C_{\alpha} t^{\alpha_{2} / 2} E\left\{\left(1+\left|x_{s}\right|^{\alpha_{1}}\right)\left|p\left[\left(x_{i}-x_{t}\right) / h\right]\right|\left|p_{\left[\alpha_{2}\right]+1}\left[\left(x_{i}-x_{s}\right) / h\right]\right|\right\} \\
:= & I_{1 n}+I_{2 n}+I_{3 n} .
\end{aligned}
$$

It follows from (8.8) with $p(x)=\left|p\left(x-x_{t} / h\right)\right|\left|p\left(x-x_{s} / h\right)\right|$ that

$$
\begin{aligned}
\Xi_{t} & :=E\left(\left|p\left[\left(x_{i}-x_{t}\right) / h\right]\right|\left|p\left[\left(x_{i}-x_{s}\right) / h\right]\right| \mid \mathcal{F}_{t}\right) \\
& \leq \frac{C h}{\sqrt{i-t}} \int_{-\infty}^{\infty}\left|p\left(x-x_{t} / h\right)\right|\left|p\left(x-x_{s} / h\right)\right| d x \\
& =\frac{C h}{\sqrt{i-t}} \int_{-\infty}^{\infty}|p(x)|\left|p\left[x+\left(x_{t}-x_{s}\right) / h\right]\right| d x .
\end{aligned}
$$

Similarly, uniformly for $x \in R$, it follows from (8.8) first and then (8.23) that

$$
\begin{aligned}
& E\left\{\left(1+\left|x_{s}\right|^{\alpha_{1}}\right)\left[1+\left(\left|x_{s}\right| / \sqrt{t}\right)^{\left[\alpha_{2}\right]+1}\right]\left|p\left(x+\left(x_{t}-x_{s}\right) / h\right)\right|\right\} \\
\leq & \frac{C h}{\sqrt{t-s}} \int_{-\infty}^{\infty}|p(x+y)| d y E\left\{\left(1+\left|x_{s}\right|^{\alpha_{1}}\right)\left[1+\left(\left|x_{s}\right| / \sqrt{t}\right)^{\left[\alpha_{2}\right]+1}\right]\right\} \\
\leq & \frac{C_{1} h s^{\alpha / 2}}{\sqrt{t-s}} .
\end{aligned}
$$


By virtue of these facts, it is readily seen that

$$
\begin{aligned}
I_{1 n} & =C_{\alpha} t^{\alpha_{2} / 2} E\left\{\left(1+\left|x_{s}\right|^{\alpha_{1}}\right)\left[1+\left(\left|x_{s}\right| / \sqrt{t}\right)^{\left[\alpha_{2}\right]+1}\right] \Xi_{t}\right\} \\
& \leq \frac{C h t^{\alpha_{2} / 2}}{\sqrt{i-t}} \int_{-\infty}^{\infty}|p(x)| E\left\{\left(1+\left|x_{s}\right|^{\alpha_{1}}\right)\left[1+\left(\left|x_{s}\right| / \sqrt{t}\right)^{\left[\alpha_{2}\right]+1}\right]\left|p\left(x+\left(x_{t}-x_{s}\right) / h\right)\right|\right\} d x \\
& \leq \frac{C_{1} h^{2}}{\sqrt{i-t}} \frac{s^{\alpha_{1} / 2} t^{\alpha_{2} / 2}}{\sqrt{t-s}} .
\end{aligned}
$$

Similarly, we have

$$
I_{2 n}+I_{3 n} \leq \frac{C h^{2}}{\sqrt{i-t}} \frac{s^{\alpha_{1} / 2} t^{\alpha_{2} / 2}}{\sqrt{t-s}} .
$$

Taking these estimates into (8.29), we obtain the required (8.27). The proof of Lemma 8.5 is now complete.

LEMMA 8.6. Let $A_{k}$ and $B_{k}$ be two sequence of random variables satisfying $A_{k} \rightarrow 0$,a.s. and $\left|A_{k}\right| \leq C, \sum_{k=1}^{n} E\left|B_{n}\right| \leq \Lambda_{n}^{2}$ and for any $c_{n} / n \rightarrow 0, \sum_{k=1}^{c_{n}} E\left|B_{n}\right|=o\left(\Lambda_{n}^{2}\right)$. Then,

$$
E\left|\sum_{k=1}^{n} A_{n} B_{n}\right|^{1 / 2}=o\left(\Lambda_{n}\right)
$$

Proof. Note that $(|x|+|y|)^{1 / 2} \leq|x|^{1 / 2}+|y|^{1 / 2}$ and

$$
\left|\sum_{k=1}^{n} A_{n} B_{n}\right| \leq C \sum_{k=1}^{\sqrt{n}}\left|B_{n}\right|+\max _{\sqrt{n} \leq k \leq n}\left|A_{k}\right| \sum_{k=1}^{n}\left|B_{n}\right| .
$$

It follows that

$$
\begin{aligned}
E\left|\sum_{k=1}^{n} A_{n} B_{n}\right|^{1 / 2} & \leq C\left(\sum_{k=1}^{\sqrt{n}} E\left|B_{n}\right|\right)^{1 / 2}+\left(E \max _{\sqrt{n} \leq k \leq n}\left|A_{k}\right|\right)^{1 / 2}\left(\sum_{k=1}^{n} E\left|B_{n}\right|\right)^{1 / 2} \\
& =o\left(\Lambda_{n}\right),
\end{aligned}
$$

as, by the dominated convergence theorem,

$$
\lim _{n \rightarrow \infty} E \max _{\sqrt{n} \leq k \leq n}\left|A_{k}\right|=E \lim _{n \rightarrow \infty} \max _{\sqrt{n} \leq k \leq n}\left|A_{k}\right|=0
$$

This proves Lemma 8.6.

8.2 Proof of Proposition 6.1. Recall $x_{s}$ is $\mathcal{F}_{s}$-measurable. By Lemma 8.4, we have

$$
\begin{aligned}
& E\left\{\left[g^{2}\left(u_{s+1}\right)+g_{1}^{2}\left(u_{s+1}\right)\right]\left(1+\left|x_{s}\right|^{\alpha}\right)\right\} \\
\leq & E\left[\left(1+\left|x_{s}\right|^{\alpha}\right) E\left\{\left[g^{2}\left(u_{s+1}\right)+g_{1}^{2}\left(u_{s+1}\right)\right] \mid \mathcal{F}_{s}\right\}\right] \\
\leq & C E\left(1+\left|x_{s}\right|^{\alpha}\right) \leq C_{1} s^{\alpha / 2} .
\end{aligned}
$$


This, together with $|p(x)| \leq C$ and (8.28), implies that

$$
\begin{aligned}
E \Lambda_{n} & \leq C \sum_{s=1}^{n-1} E g\left(u_{s+1}\right) g_{1}\left(u_{s+2}\right)\left(1+\left|x_{s}\right|^{\alpha_{1}}\right)\left(1+\left|x_{s+1}\right|^{\alpha_{2}}\right)+C \sum_{\substack{s, t=1 \\
s \neq t, t+1}}^{n} \frac{h s^{\alpha_{1} / 2} t^{\alpha_{2} / 2}}{\sqrt{|t-s|}} \\
& \leq C \sum_{s=1}^{n-1}\left\{E g^{2}\left(u_{s+1}\right)\left(1+\left|x_{s}\right|^{2 \alpha_{1}}\right)\right\}^{1 / 2}\left\{E g_{1}^{2}\left(u_{s+2}\right)\left(1+\left|x_{s+1}\right|^{2 \alpha_{2}}\right)\right\}^{1 / 2}+C n^{3 / 2+\alpha_{1} / 2+\alpha_{2} / 2} h \\
& =C \sum_{s=1}^{n-1} s^{\alpha_{1} / 2}(s+1)^{\alpha_{2} / 2}+C n^{3 / 2+\alpha_{1} / 2+\alpha_{2} / 2} h \\
& \leq C_{1} n^{3 / 2+\alpha_{1} / 2+\alpha_{2} / 2} h,
\end{aligned}
$$

since $n h^{2} \rightarrow \infty$. This proves $\Lambda_{n}=O_{P}\left(n^{3 / 2+\alpha_{1} / 2+\alpha_{2} / 2} h\right)$. Similarly it follows from (8.25) and (8.26) that

$$
\begin{aligned}
E \widetilde{\Lambda}_{n} & \leq C h^{1 / 2} \sum_{s=1}^{n-1}\left(1+s^{\left(\alpha_{1}-1\right) / 2}\right)+C h \sum_{s=1}^{n-1}\left(1+s^{\left(\alpha_{1}-1\right) / 2}\right) \sum_{t=s+2}^{n} 1 / \sqrt{t-s} \\
& \leq C n^{\max \left\{3 / 2,1+\alpha_{1} / 2\right\}} h,
\end{aligned}
$$

which yields that $\widetilde{\Lambda}_{n}=O_{P}\left(n^{\max \left\{3 / 2,1+\alpha_{1} / 2\right\}} h\right)$. The proof of Proposition 6.1 is now complete.

8.3 Proof of Proposition 6.2. For the convenience in notation, we use $g(x)$ instead of $g(x, \theta)$. We may write

$$
\begin{aligned}
\Delta_{n} & =\sum_{s=2}^{n} u_{s+1} \sum_{t=1}^{s-1} g\left(x_{t}\right) K\left[\left(x_{t}-x_{s}\right) / h\right]+\sum_{s=1}^{n-1} u_{s+1} \sum_{t=s+1}^{n} g\left(x_{t}\right) K\left[\left(x_{t}-x_{s}\right) / h\right] \\
& :=\Delta_{1 n}+\Delta_{2 n} .
\end{aligned}
$$

By recalling $x_{1}, \ldots, x_{t}$ are $\mathcal{F}_{t}$-measurable, it follows from $(8.27)-(8.28)$ with $p(x)=K(-x)$ that

$$
\begin{aligned}
E \Delta_{1 n}^{2} \leq & C \sum_{i=1}^{n} E\left\{\left(\sum_{t=1}^{i-1} g\left(x_{t}\right) K\left[\left(x_{t}-x_{i}\right) / h\right]\right)^{2} E\left(u_{t+1}^{2} \mid \mathcal{F}_{t}\right)\right\} \\
\leq & C_{1} \sum_{i=1}^{n} \sum_{1 \leq s<t \leq i-1} E\left\{\left(1+\left|x_{s}\right|^{\beta}\right)\left(1+\left|x_{t}\right|^{\beta}\right) K\left[\left(x_{t}-x_{i}\right) / h\right] K\left[\left(x_{s}-x_{i}\right) / h\right]\right\} \\
& +C_{1} \sum_{i=1}^{n} \sum_{t=1}^{i-1} E\left\{\left(1+\left|x_{t}\right|^{2 \beta}\right) K^{2}\left[\left(x_{t}-x_{i}\right) / h\right]\right\} \\
\leq & C_{2} h^{2} \sum_{i=1}^{n} \sum_{1 \leq s<t \leq i-1} \frac{s^{\beta / 2} t^{\beta / 2}}{\sqrt{i-t} \sqrt{t-s}}+C_{2} h \sum_{i=1}^{n} \sum_{t=1}^{i-1} \frac{t^{\beta}}{\sqrt{i-t}} \\
\leq & C_{3}\left(h^{2} n^{2+\beta}+h n^{3 / 2+\beta}\right) \leq C n^{2+\beta} h^{2},
\end{aligned}
$$


since $n h^{2} \rightarrow \infty$. This yields

$$
\Delta_{1 n}=O_{P}\left(n^{5 / 4+\beta / 2} h^{3 / 4}\right)
$$

As for $\Delta_{2 n}$, we may write

$$
\Delta_{2 n}=\Delta_{2 n 1}+\sum_{i=1}^{n} Z_{2 i}+\sum_{i=1}^{n} u_{i+1} g\left(x_{i}\right) K\left[\left(x_{i+1}-x_{i}\right) / h\right]
$$

where $Z_{2 i}=u_{i+1} g\left(x_{i}\right) \sum_{t=i+2}^{n} K\left[\left(x_{t}-x_{i}\right) / h\right]$, and

$$
\left|\Delta_{2 n 1}\right| \leq \sum_{s=1}^{n-1}\left|u_{s+1}\right| \sum_{t=s+1}^{n}\left|g\left(x_{t}\right)-g\left(x_{s}\right)\right| K\left[\left(x_{t}-x_{s}\right) / h\right] .
$$

Let $\beta^{\prime}=\beta$ if $\beta>0$ and $\beta^{\prime}=\gamma^{\prime}$ if $\beta=0$. Similarly to the proof of (6.26), it follows from (2.5) and Proposition 6.1 that

$$
\begin{aligned}
\left|\Delta_{2 n 1}\right| & \leq C h^{\gamma} \sum_{1 \leq s<t \leq n}\left|u_{s+1}\right|\left(1+\left|x_{s}\right|^{\beta^{\prime}}+\left|x_{t}-x_{s}\right|^{\beta}\right) K_{\gamma}\left[\left(x_{t}-x_{s}\right) / h\right] \\
& =O_{P}\left(h^{1+\gamma} n^{\max \left\{3 / 2,1+\beta^{\prime} / 2\right\}}\right),
\end{aligned}
$$

where $K_{u}(x)=|x|^{u} K(x), u>0$. Recalling $n h^{4} \rightarrow 0$ and $1 \geq \gamma>\max \{0,3 / 4-2 \beta\}$, simple calculation shows that $\Delta_{2 n 1}=O_{P}\left(n^{5 / 4+\beta / 2} h^{3 / 4}\right)$. On the other hand, the same arguments in the proof of (8.32) show that

$$
\begin{aligned}
& \sum_{i=1}^{n} E\left|u_{i+1}\right|\left|g\left(x_{i}\right)\right| K\left[\left(x_{i+1}-x_{i}\right) / h\right] \\
\leq & \sum_{i=1}^{n}\left(E\left|u_{i+1}\right|^{2}\left|g\left(x_{i}\right)\right|^{2}\right)^{1 / 2}\left(E K^{2}\left[\left(x_{i+1}-x_{i}\right) / h\right]\right)^{1 / 2} \\
\leq & C \sum_{i=1}^{n} i^{\beta / 2} \sqrt{h} \leq C_{1} n^{\beta / 2+1} \sqrt{h},
\end{aligned}
$$

which implies $\sum_{i=1}^{n} u_{i+1} g\left(x_{i}\right) K\left[\left(x_{i+1}-x_{i}\right) / h\right]=O_{P}\left(n^{5 / 4+\beta / 2} h^{3 / 4}\right)$, since $n h^{2} \rightarrow \infty$. Combining all facts above, the result (6.3) will follow if we prove $\sum_{i=1}^{n} Z_{2 i}=O_{P}\left(n^{5 / 4+\beta / 2} h^{3 / 4}\right)$. In this regard, it only need to show that

$$
\begin{aligned}
E Z_{2 i}^{2} & \leq C n h^{2} i^{\beta}, \\
E\left(Z_{2 i} Z_{2 j}\right) & \leq C \sqrt{n} h^{3 / 2}\left(1+h^{1 / 2} \log n\right) i^{\beta / 2} j^{\beta / 2},
\end{aligned}
$$

for $i<j$. Indeed, by (8.35)-(8.36), it is readily seen that

$$
\begin{aligned}
E\left(\sum_{i=1}^{n} Z_{2 i}\right)^{2} & \leq C n h^{2} \sum_{i=1}^{n} i^{\beta}+C \sqrt{n} h^{3 / 2}\left(1+h^{1 / 2} \log n\right) \sum_{1 \leq i<j \leq n-1} i^{\beta / 2} j^{\beta / 2} \\
& \leq C n^{5 / 2+\beta} h^{3 / 2}
\end{aligned}
$$


since $n h^{2} \rightarrow \infty$ and $h \log ^{2} n \rightarrow 0$. This implies $\sum_{i=1}^{n} Z_{2 i}=O_{P}\left(n^{5 / 4+\beta / 2} h^{3 / 4}\right)$.

We next prove (8.35) and (8.36). To this end, write

$$
J(i, j, s, t)=E\left[u_{i+1} u_{j+1} g\left(x_{i}\right) g\left(x_{j}\right) K\left[\left(x_{s}-x_{i}\right) / h\right] K\left[\left(x_{t}-x_{j}\right) / h\right]\right] .
$$

If $i=j$ and $s=t \geq i+2$, by recalling $\sup _{i \geq 1} E\left(u_{i+1}^{4} \mid \mathcal{F}_{i}\right)<\infty$ and $\sup _{x} K(x)<\infty$, it follows from (8.28) that

$$
\begin{aligned}
J(i, i, t, t) & \leq \sup _{x} K(x) E\left\{u_{i+1}^{2}\left(1+\left|x_{i}\right|^{2 \beta}\right) K\left[\left(x_{t}-x_{i}\right) / h\right]\right\} \\
& \leq \frac{C h i^{\beta}}{\sqrt{t-i}} .
\end{aligned}
$$

Similarly, if $i=j$ and $i+2 \leq s<t$, then by (8.8) and (8.28)

$$
\begin{aligned}
J(i, i, s, t) & =E\left\{u_{i+1}^{2} g\left(x_{i}\right)^{2} K\left[\left(x_{s}-x_{i}\right) / h\right] E\left(K\left[\left(x_{t}-x_{i}\right) / h\right] \mid \mathcal{F}_{s}\right)\right\} \\
& \leq \frac{C h}{\sqrt{t-s}} E\left\{u_{i+1}^{2}\left(1+\left|x_{i}\right|^{2 \beta}\right) K\left[\left(x_{s}-x_{i}\right) / h\right]\right\} \\
& \leq \frac{C h^{2} i^{\beta}}{\sqrt{t-s}} \frac{1}{\sqrt{s-i}} .
\end{aligned}
$$

By virtue of these estimates, it is readily seen that

$$
\begin{aligned}
E Z_{2 i}^{2} & =2 \sum_{i+2 \leq s<t \leq n} J(i, i, s, t)+\sum_{t=i+2}^{n} J(i, i, t, t) \\
& \leq C h^{2} \sum_{i+2 \leq s<t \leq n} \frac{1}{\sqrt{t-s}} \frac{1}{\sqrt{s-i}} i^{\beta}+C h \sum_{t=i+2}^{n} \frac{1}{\sqrt{t-i}} i^{\beta} \\
& \leq C\left(n h^{2}+\sqrt{n} h\right) i^{\beta} \leq C n h^{2} i^{\beta},
\end{aligned}
$$

since $n h^{2} \rightarrow \infty$, which yields (8.35).

To prove (8.36), for $i<j$ and $m_{0}=1+[\beta]$, write $\Omega=\{s: s=j+1$ or $i+1 \leq s \leq$ $\left.i+m_{0}+1\right\}$ and assume $\sum_{i=k}^{s}=0$ if $s<k$. We have

$$
\begin{aligned}
E\left(Z_{2 i} Z_{2 j}\right) & =\left(\sum_{s \in \Omega} \sum_{t=j+2}^{n}+\sum_{s=i+m_{0}+2}^{j} \sum_{t=j+2}^{n}+\sum_{s=j+2}^{n} \sum_{t=j+2}^{n}\right) J(i, j, s, t) \\
& :=A_{1 i}+A_{2 i}+A_{3 i} .
\end{aligned}
$$

First calculate $A_{2 i}$. For $i<j, i+m_{0}+2 \leq s \leq j$ and $t \geq j+2$, it follows from (8.7) with $p(x)=K\left(x-x_{j} / h\right)$ that

$$
\begin{aligned}
J(i, j, s, t) & \leq E\left\{\left|u_{i+1}\right|\left|g\left(x_{i}\right)\right|\left|g\left(x_{j}\right)\right| K\left[\left(x_{s}-x_{i}\right) / h\right]\left|E\left(u_{j+1} K\left[\left(x_{t}-x_{j}\right) / h\right] \mid \mathcal{F}_{j}\right)\right|\right\} \\
& \leq \frac{C h}{t-j} E\left\{\left|u_{i+1} \| g\left(x_{i}\right)\right|\left|g\left(x_{j}\right)\right| K\left[\left(x_{s}-x_{i}\right) / h\right]\right\} .
\end{aligned}
$$


Furthermore, by recalling $|g(x)| \leq C\left(1+|x|^{\beta}\right)$ for some $\beta \geq 0$, the result (8.24) implies that

$$
\begin{aligned}
& E\left\{\left|u_{i+1}\right|\left|g\left(x_{i}\right)\right|\left|g\left(x_{j}\right)\right| K\left[\left(x_{s}-x_{i}\right) / h\right]\right\} \\
\leq & C E\left[\left|u_{i+1}\right|\left(1+\left|x_{i}\right|^{\beta}\right) K\left[\left(x_{s}-x_{i}\right) / h\right] E\left\{\left(1+\left|x_{j}\right|^{\beta}\right) \mid \mathcal{F}_{s}\right\}\right] \\
\leq & C_{1}(j-s)^{\beta / 2} E\left\{\left|u_{i+1}\right|\left(1+\left|x_{i}\right|^{\beta}\right) K\left[\left(x_{s}-x_{i}\right) / h\right]\right\} \\
& +C_{1} E\left\{\left|u_{i+1}\right|\left(1+\left|x_{i}\right|^{\beta} \mid\right)\left|x_{s}\right|^{\beta} K\left[\left(x_{s}-x_{i}\right) / h\right]\right\} \\
& +C_{1} E\left\{\left|u_{i+1}\right|\left(1+\left|x_{i}\right|^{\beta} \mid\right)\left(\sum_{k=0}^{\infty}(k+1)^{-1-\delta}\left|\epsilon_{s-k}\right|\right)^{\beta} K\left[\left(x_{s}-x_{i}\right) / h\right]\right\} \\
:= & B_{i 1}+B_{i 2}+B_{i 3} .
\end{aligned}
$$

It follows from (8.28) that

$$
B_{i 1}+B_{i 2} \leq \frac{C h}{\sqrt{s-i}} i^{\beta / 2} j^{\beta / 2}
$$

To calculate $B_{i 3}$, first notice that

$$
\begin{aligned}
\left(\sum_{k=0}^{\infty}(k+1)^{-1-\delta}\left|\epsilon_{s-k}\right|\right)^{\beta} & \leq 1+\left(\sum_{k=0}^{\infty}(k+1)^{-1-\delta}\left|\epsilon_{s-k}\right|\right)^{[\beta]+1} \\
& =1+\sum_{k_{1}, \ldots, k_{m_{0}}=0}^{\infty} \prod_{u=1}^{m_{0}}\left(k_{u}+1\right)^{-1-\delta} \prod_{u=1}^{m_{0}}\left|\epsilon_{t-k_{u}}\right|,
\end{aligned}
$$

and by using (8.10) in Lemma 8.1 with $p(x)=K\left(x-x_{i} / h\right)$,

$$
\begin{aligned}
& E\left\{\left|u_{i+1}\right|\left(1+\left|x_{i}\right|^{\beta} \mid\right) \prod_{u=1}^{m_{0}}\left|\epsilon_{s-k_{u}}\right| K\left[\left(x_{s}-x_{i}\right) / h\right]\right\} \\
\leq & E\left\{\left(1+\left|x_{i}\right|^{\beta} \mid\right) \prod_{\substack{u=1 \\
s-k_{u} \leq i}}^{m_{0}}\left|\epsilon_{s-k_{u}}\right| E\left(\left|u_{i+1}\right| \prod_{\substack{u=1 \\
s \geq s-k_{u} \geq i+1}}^{m_{0}}\left|\epsilon_{s-k_{u}}\right| K\left[\left(x_{s}-x_{i}\right) / h\right] \mid \mathcal{F}_{i}\right)\right\} \\
\leq & \frac{C h}{\sqrt{s-i-m_{0}}} E\left\{\left(1+\left|x_{i}\right|^{\beta} \mid\right) \prod_{\substack{u=1 \\
s-k_{u} \leq i}}^{m_{0}}\left|\epsilon_{s-k_{u}}\right|\right\} \\
\leq & \frac{C h}{\sqrt{s-i}}\left[E\left(1+\left|x_{i}\right|^{\beta} \mid\right)^{2}\right]^{1 / 2}\left(E \prod_{\substack{u=1 \\
s-k_{u} \leq i}}^{m_{0}}\left|\epsilon_{s-k_{u}}\right|^{2}\right)^{1 / 2} \\
\leq & \frac{C h}{\sqrt{s-i}} i^{\beta / 2} .
\end{aligned}
$$


These facts imply that

$$
\begin{aligned}
B_{i 3} \leq & C E\left[\left|u_{i+1}\right|\left(1+\left|x_{i}\right|^{\beta} \mid\right)\left\{1+\left(\sum_{k=0}^{\infty}(k+1)^{-1-\delta}\left|\epsilon_{t-k}\right|\right)^{1+[\beta]}\right\} K\left[\left(x_{t}-x_{i}\right) / h\right]\right] \\
\leq & C E\left\{\left|u_{i+1}\right|\left(1+\left|x_{i}\right|^{\beta} \mid\right) K\left[\left(x_{s}-x_{i}\right) / h\right]\right\} \\
& +\sum_{k_{1}, \ldots, k_{m_{0}}}^{\infty} \prod_{u=1}^{m_{0}}\left(k_{u}+1\right)^{-1-\delta} E\left\{\left|u_{i+1}\right|\left(1+\left|x_{i}\right|^{\beta} \mid\right) \prod_{u=1}^{m_{0}}\left|\epsilon_{s-k_{u}}\right| K\left[\left(x_{s}-x_{i}\right) / h\right]\right\} \\
\leq & \frac{C h}{\sqrt{s-i}} i^{\beta / 2} .
\end{aligned}
$$

Combining (8.38)-(8.41), we get

$$
\begin{aligned}
A_{2 i} & \leq \sum_{s=i+m+2}^{j} \sum_{t=j+2}^{n} \frac{C h}{t-j} \frac{C_{1} h}{\sqrt{s-i}} i^{\beta / 2} j^{\beta / 2} \\
& \leq C \sqrt{n} h^{2} \log n i^{\beta / 2} j^{\beta / 2} .
\end{aligned}
$$

Next calculate $A_{1 i}$. For $i<j, s \in \Omega$ and $t \geq j+2$, similar arguments to those above show that

$$
\begin{aligned}
& |J(i, j, s, t)| \\
\leq & E\left[\left|u_{i+1}\right|\left|u_{j+1}\right|\left|g\left(x_{i}\right)\right|\left|g\left(x_{j}\right)\right| K\left[\left(x_{s}-x_{i}\right) / h\right]\left|E\left(K\left[\left(x_{t}-x_{j}\right) / h\right] \mid \mathcal{F}_{j+1}\right)\right|\right] \\
\leq & \frac{C h}{\sqrt{t-j}} E\left[\left|u_{i+1}\right|\left|u_{j+1}\right|\left|g\left(x_{i}\right)\right|\left|g\left(x_{j}\right)\right| K\left[\left(x_{s}-x_{i}\right) / h\right]\right] \\
\leq & \frac{C h}{\sqrt{t-j}}\left\{E\left(\left|u_{i+1}\right|^{2}\left|g\left(x_{i}\right)\right|^{2} K^{2}\left[\left(x_{s}-x_{i}\right) / h\right]\right)\right\}^{1 / 2}\left\{E\left[\left|u_{j+1}^{2} g^{2}\left(x_{j}\right)\right|\right]\right\}^{1 / 2} \\
\leq & \frac{C h}{\sqrt{t-j}} \frac{h^{1 / 2}}{(s-i)^{1 / 4}}\left[E\left(1+\left|x_{i}\right|^{2 \beta}\right)\right]^{1 / 2}\left[E\left(1+\left|x_{j}\right|^{2 \beta}\right)\right]^{1 / 2} \\
\leq & \frac{C h}{\sqrt{t-j}} \frac{h^{1 / 2}}{(s-i)^{1 / 4}} i^{\beta / 2} j^{\beta / 2} .
\end{aligned}
$$

This yields

$$
\begin{aligned}
A_{1 i} & \leq C h^{3 / 2} i^{\beta / 2} j^{\beta / 2} \sum_{t=j+2}^{n} \frac{1}{\sqrt{t-j}} \sum_{s=i+1}^{i+m_{0}+1} \frac{1}{(s-i)^{1 / 4}} \\
& \leq C m_{0} \sqrt{n} h^{3 / 2} i^{\beta / 2} j^{\beta / 2} .
\end{aligned}
$$


Finally, we calculate $A_{3 i}$. It follows from (8.6) that, for $i<j, j+2 \leq s<t \leq n$,

$$
\begin{aligned}
& J(i, j, s, t) \\
= & E\left[u_{i+1} u_{j+1} g\left(x_{i}\right) g\left(x_{j}\right) K\left[\left(x_{s}-x_{i}\right) / h\right] E\left\{K\left[\left(x_{t}-x_{j}\right) / h\right] \mid \mathcal{F}_{s}\right\}\right] \\
= & \frac{h}{\sqrt{t-s}} \int_{-\infty}^{\infty} E\left\{u_{i+1} u_{j+1} g\left(x_{i}\right) g\left(x_{j}\right) K\left[\left(x_{s}-x_{i}\right) / h\right] K\left[\left(\rho^{t-s} x_{s}-x_{j}\right) / h+x\right]\right\} \\
& h_{s, t}\left(\frac{h x}{\sqrt{t-s}}\right) d x+C_{1 n} \\
:= & C_{n}+C_{1 n},
\end{aligned}
$$

where

$$
\left|C_{1 n}\right| \leq \frac{C h}{t-s} E\left\{\left|u_{i+1}\right|\left|u_{j+1}\right|\left|g\left(x_{i}\right) g\left(x_{j}\right)\right| K\left[\left(x_{s}-x_{j}\right) / h\right]\left(1+\sum_{k=0}^{\infty}(k+1)^{-1-\delta}\left|\epsilon_{s-k}\right|\right)\right\} .
$$

Note that, by (8.10) and Lemma 8.4,

$$
\begin{aligned}
& J_{k}^{*}(i, j, s) \\
:= & E\left\{\left|u_{i+1}\right|\left|u_{j+1}\right|\left|g\left(x_{i}\right) g\left(x_{j}\right)\right| K\left[\left(x_{s}-x_{j}\right) / h\right]\left(1+\left|\epsilon_{s-k}\right|\right)\right\} \\
= & E\left\{\left|u_{i+1}\right|\left|g\left(x_{i}\right) g\left(x_{j}\right)\right| E\left[\left|u_{j+1}\right| K\left[\left(x_{s}-x_{j}\right) / h\right]\left(1+\left|\epsilon_{s-k}\right|\right) \mid \mathcal{F}_{j}\right]\right\} \\
\leq & \frac{C h}{\sqrt{s-j}} E\left\{\left|u_{i+1}\right|\left|g\left(x_{i}\right) g\left(x_{j}\right)\right|\right\} \\
\leq & \frac{C h}{\sqrt{s-j}}\left[E\left\{u_{i+1}^{2}\left(1+\left|x_{i}\right|^{2 \beta}\right)\right\}\right]^{1 / 2}\left[E\left(1+\left|x_{j}\right|^{2 \beta}\right)\right]^{1 / 2} \\
\leq & \frac{C h}{\sqrt{s-j}} i^{\beta} j^{\beta / 2}, \quad \text { for } s-k \geq j+1
\end{aligned}
$$

and similarly,

$$
\begin{aligned}
J_{k}^{*}(i, j, s) & =E\left\{\left|u_{i+1}\right|\left|g\left(x_{i}\right) g\left(x_{j}\right)\right|\left(1+\left|\epsilon_{s-k}\right|\right) E\left[\left|u_{j+1}\right| K\left[\left(x_{s}-x_{j}\right) / h\right] \mid \mathcal{F}_{j}\right]\right\} \\
& \leq \frac{C h}{\sqrt{s-j}} E\left\{\left|u_{i+1}\right|\left|g\left(x_{i}\right) g\left(x_{j}\right)\right|\left(1+\left|\epsilon_{s-k}\right|\right)\right\} \\
& \leq \frac{C h}{\sqrt{s-j}}\left[E\left\{u_{i+1}^{2}\left(1+\left|x_{i}\right|^{2 \beta}\right)\right\}\right]^{1 / 2}\left[E\left(1+\left|x_{j}\right|^{4 \beta}\right)\right]^{1 / 4}\left\{E\left(1+\left|\epsilon_{s-k}\right|\right)^{4}\right\}^{1 / 4} \\
& \leq \frac{C h}{\sqrt{s-j}} i^{\beta} j^{\beta / 2}, \quad \text { for } s-k \leq j
\end{aligned}
$$

By virtue of these estimates, it is readily seen that

$$
\left|C_{1 n}\right| \leq \frac{C h}{t-s} \sum_{k=0}^{\infty}(k+1)^{-1-\delta} J_{k}^{*}(i, j, s, t) \leq \frac{C h^{2}}{t-s} \frac{1}{\sqrt{s-j}} i^{\beta} j^{\beta / 2}
$$


On the other hand, by noting that (8.7) with $p(y)=K\left(y-x_{j} / h\right) K\left(\rho^{t-s} y-x_{i} / h+x\right)$ yields that

$$
\begin{aligned}
& \left|E\left\{u_{j+1} K\left[\left(x_{s}-x_{j}\right) / h\right] K\left[\left(\rho^{t-s} x_{s}-x_{i}\right) / h+x\right] \mid \mathcal{F}_{j}\right\}\right| \\
& \leq \frac{C h}{s-j} \int_{-\infty}^{\infty} K\left(y-x_{j} / h\right) K\left(\rho^{t-s} y-x_{i} / h+x\right) d y \leq \frac{C_{1} h}{s-j}
\end{aligned}
$$

the conditional argument implies that

$$
\begin{aligned}
\left|C_{n}\right| & \leq \frac{C h^{2}}{\sqrt{t-s}} \frac{1}{s-j} E\left\{\left|u_{i+1}\right|\left|g\left(x_{i}\right) g\left(x_{j}\right)\right|\right\} \\
& \leq \frac{C h^{2}}{\sqrt{t-s}} \frac{1}{s-j}\left[E\left\{u_{i+1}^{2}\left(1+\left|x_{i}\right|^{2 \beta}\right)\right\}\right]^{1 / 2}\left[E\left(1+\left|x_{j}\right|^{2 \beta}\right)\right]^{1 / 2} \\
& \leq \frac{C h^{2}}{\sqrt{t-s}} \frac{1}{s-j} i^{\beta / 2} j^{\beta / 2} .
\end{aligned}
$$

It follows from (8.44)-(8.46) that, for $i<j$ and $j+2 \leq s<t \leq n$,

$$
|J(i, j, s, t)| \leq c h^{2}\left(\frac{1}{\sqrt{t-s}} \frac{1}{s-j}+\frac{1}{t-s} \frac{1}{\sqrt{s-j}}\right) i^{\beta / 2} j^{\beta / 2} .
$$

Similarly, for $i<j$ and $j+2 \leq s=t \leq n$, we have

$$
\begin{aligned}
|J(i, j, t, t)| & =E\left\{\left|u_{i+1}\right|\left|g\left(x_{i}\right) g\left(x_{j}\right)\right|\left|E\left(u_{j+1} K\left[\left(x_{t}-x_{i}\right) / h\right] K\left[\left(x_{t}-x_{j}\right) / h\right] \mid \mathcal{F}_{j}\right)\right|\right\} \\
& \leq \frac{C h}{t-j} E\left\{\left|u_{i+1}\right|\left|g\left(x_{i}\right) g\left(x_{j}\right)\right|\right\} \\
& \leq \frac{C h}{t-j} i^{\beta / 2} j^{\beta / 2} .
\end{aligned}
$$

It is now readily seen that

$$
\begin{aligned}
\left|A_{3 i}\right| & \leq \sum_{t=j+2}^{n}|J(i, j, t, t)|+2 \sum_{j+2 \leq s<t \leq n}|J(i, j, s, t)| \\
& \leq C i^{\beta / 2} j^{\beta / 2}\left[\sum_{t=j+2}^{n} \frac{h}{t-j}+\sum_{j+2 \leq s<t \leq n}\left(\frac{h^{2}}{\sqrt{t-s}} \frac{1}{s-j}+\frac{1}{t-s} \frac{1}{\sqrt{s-j}}\right)\right] \\
& \leq C \sqrt{n} h^{2} \log n i^{\beta / 2} j^{\beta / 2},
\end{aligned}
$$

since $n h^{2} \rightarrow \infty$. The result (8.36) follows from (8.37), (8.42), (8.43) and (8.47). The proof of Proposition 6.2 is now complete.

8.4 Proof of Proposition 6.3. We may write

$$
\begin{aligned}
\Gamma_{n}= & \sum_{t=1}^{n}\left(E\left(u_{t+1}^{2} \mid \mathcal{F}_{t}-\sigma^{2}\right) \sum_{\substack{s=1 \\
s \neq t}}^{n} g\left(u_{s+1}\right) K^{2}\left[\left(x_{t}-x_{s}\right) / h\right]\right. \\
& +\sum_{\substack{s, t=1 \\
s \neq t}}^{n} g\left(u_{s+1}\right)\left[u_{t+1}^{2}-E\left(u_{t+1}^{2} \mid \mathcal{F}_{t}\right)\right] K^{2}\left[\left(x_{t}-x_{s}\right) / h\right] \\
:= & \Gamma_{1 n}+\Gamma_{2 n} .
\end{aligned}
$$


Recall that, by (8.28) in Lemma 8.5,

$$
E\left\{g\left(u_{s+1}\right) K^{2}\left[\left(x_{t}-x_{s}\right) / h\right]\right\} \leq \frac{C h}{\sqrt{|t-s|}} .
$$

It is readily seen that

$$
\sum_{t=1}^{n} \sum_{\substack{s=1 \\ s \neq t}}^{n} E\left\{g\left(u_{s+1}\right) K^{2}\left[\left(x_{t}-x_{s}\right) / h\right]\right\} \leq C n^{3 / 2} h
$$

and for any $c_{n} / n \rightarrow 0$,

$$
\sum_{t=1}^{c_{n}} \sum_{\substack{s=1 \\ s \neq t}}^{n} E\left\{g\left(u_{s+1}\right) K^{2}\left[\left(x_{t}-x_{s}\right) / h\right]\right\} \leq C c_{n} \sqrt{n} h=o\left(n^{3 / 2} h\right) .
$$

By virtue of these facts and $E\left(u_{s+1}^{2} \mid \mathcal{F}_{s}\right) \rightarrow \sigma^{2}$,a.s., it follows from Lemma 8.6 that

$$
E\left|\Gamma_{1 n}\right|^{1 / 2}=o\left(n^{3 / 4} \sqrt{h}\right)
$$

which implies that $\Gamma_{1 n}=o_{P}\left(n^{3 / 2} h\right)$.

As for $\Gamma_{2 n}$, we may write

$$
\begin{aligned}
\Gamma_{2 n}= & \sum_{s=2}^{n} g\left(u_{s+1}\right) \sum_{t=1}^{s-1}\left[u_{t+1}^{2}-E\left(u_{t+1}^{2} \mid \mathcal{F}_{t}\right)\right] K^{2}\left[\left(x_{t}-x_{s}\right) / h\right] \\
& +\sum_{t=2}^{n}\left[u_{t+1}^{2}-E\left(u_{t+1}^{2} \mid \mathcal{F}_{t}\right)\right] \sum_{s=1}^{t-1} g\left(u_{s+1}\right) I(|g| \leq \log n) K^{2}\left[\left(x_{t}-x_{s}\right) / h\right] \\
& +\sum_{t=2}^{n}\left[u_{t+1}^{2}-E\left(u_{t+1}^{2} \mid \mathcal{F}_{t}\right)\right] \sum_{s=1}^{t-1} g\left(u_{s+1}\right) I(|g|>\log n) K^{2}\left[\left(x_{t}-x_{s}\right) / h\right] \\
:= & \Gamma_{2 n 1}+\Gamma_{2 n 2}+\Gamma_{2 n 3} .
\end{aligned}
$$

By (6.7) in Proposition 6.5 and conditional argument,

$$
\begin{aligned}
E\left|\Gamma_{2 n 1}\right| & \leq \sum_{s=2}^{n} E\left\{\left|\sum_{t=1}^{s-1}\left[u_{t+1}^{2}-E\left(u_{t+1}^{2} \mid \mathcal{F}_{t}\right)\right] K^{2}\left[\left(x_{t}-x_{s}\right) / h\right]\right| E\left[\left|g\left(u_{s+1}\right)\right| \mid \mathcal{F}_{s}\right]\right\} \\
& \leq C \sum_{s=2}^{n}\left(s h^{2}\right)^{1 / 4} \leq C n^{5 / 4} \sqrt{h},
\end{aligned}
$$

which implies that $\Gamma_{2 n 1}=o_{P}\left(n^{3 / 2} h\right)$, since $n h^{2} \rightarrow \infty$. Similarly to the proof of (8.32), we have

$$
\begin{aligned}
E \Gamma_{2 n 2}^{2} & \leq C \log ^{2} n \sum_{t=2}^{n} E\left(\sum_{s=1}^{t-1} K^{2}\left[\left(x_{t}-x_{s}\right) / h\right]\right)^{2} \\
& \leq C n^{2} h^{2} \log ^{2} n
\end{aligned}
$$


On the other hand, by recalling (8.9), simple calculations show that

$$
\begin{aligned}
E\left|\Gamma_{2 n 3}\right| & \leq C \sum_{t=2}^{n} E\left|g\left(u_{t}\right)\right| I(|g|>\log n)+C \sum_{t=2}^{n} \sum_{s=1}^{t-2} \frac{h E\left(\left|g\left(u_{s+1}\right)\right| I(|g|>\log n) \mid \mathcal{F}_{s}\right)}{\sqrt{t-s}} \\
& \leq C\left(\log ^{-1} n\right)\left(n+n^{3 / 2} h\right) .
\end{aligned}
$$

By virtue of (8.51) and (8.52),

$$
\left|\Gamma_{2 n 2}\right|+\left|\Gamma_{2 n 3}\right|=O_{P}(n h \log n)+O_{P}\left(n^{3 / 2} h \log ^{-1} n\right)=o_{P}\left(n^{3 / 2} h\right)
$$

Combining all above estimates, we obtain the required (6.4), and hence completes the proof of Proposition 6.3.

8.5 Proof of Proposition 6.4. By using the convention that $\sum_{i=s}^{t}=0$ if $t<s$, let

$$
R_{1 n}=\sum_{t=1}^{n} \sum_{j=1}^{t-2} u_{t} u_{j+1} K\left[\left(x_{t}-x_{t-1}\right) / h\right] K\left[\left(x_{t}-x_{j}\right) / h\right]
$$

and $R_{2 n}=\sum_{t=1}^{n} Z_{t}$, where

$$
Z_{t}=\sum_{i=1}^{t-2} \sum_{j=1}^{i-1} u_{i+1} u_{j+1} K\left[\left(x_{t}-x_{i}\right) / h\right] K\left[\left(x_{t}-x_{j}\right) / h\right] .
$$

Recall $\left|u_{t}\right| \leq A$. It follows easily from (8.12) with $p_{1}(x)=p_{2}(x)=K(x)$ in Lemma 8.2 that

$$
\begin{aligned}
E\left|R_{1 n}\right| & \leq A^{2} \sum_{t=1}^{n} \sum_{j=1}^{t-2} E\left\{K\left[\left(x_{t}-x_{t-1}\right) / h\right] K\left[\left(x_{t}-x_{j}\right) / h\right]\right\} \\
& \leq A^{2} \sum_{t=1}^{n} \sum_{j=1}^{t-2} \frac{h^{2}}{\sqrt{t-j}} \leq C n^{3 / 2} h^{2} .
\end{aligned}
$$

To calculate $R_{2 n}$, we will show that

$$
E Z_{t}^{2} \leq C t^{3 / 2} h^{3}
$$

and for $s<t$,

$$
\begin{gathered}
E\left(Z_{s} Z_{t}\right) \leq \frac{C h^{4}}{\sqrt{t-s}}\left\{s^{2}[\log (t-s)+\log s]+\frac{s^{5 / 2}}{\sqrt{t-s}}+s(t-s) \log (t-s)\right\} \\
+\frac{C h^{3} s}{\sqrt{t-s}}
\end{gathered}
$$


By virtue of (8.54) and (8.55), we obtain

$$
\begin{aligned}
E R_{2 n}^{2} & \leq 2 \sum_{t=1}^{n} \sum_{s=1}^{t-1} E\left(Z_{t} Z_{s}\right)+\sum_{t=1}^{n} E Z_{t}^{2} \\
& \leq\left(C h^{2} \sqrt{n} \log n+C n^{-1 / 2} h\right) n^{3} h^{2}=o\left(n^{3} h^{2}\right),
\end{aligned}
$$

since $n h^{4} \log ^{2} n \rightarrow 0$. By (8.53),(8.56) and the fact that $\left|R_{n}\right| \leq 2\left|R_{1 n}\right|+2\left|R_{2 n}\right|$, we obtain

$$
E\left|R_{n}\right| \leq 2 E\left|R_{1 n}\right|+2\left(E R_{2 n}^{2}\right)^{1 / 2}=o\left(n^{3 / 2} h\right)
$$

which implies (6.5).

It suffices to show (8.54) and (8.55). The result (8.54) is simple. Indeed, by letting $Z_{t 12}=\sum_{i=1}^{t-2} u_{i+1} K\left[\left(x_{t}-x_{i}\right) / h\right]$ as in Proposition 6.5 , it is readily seen that

$$
2 Z_{t}=Z_{t 12}^{2}-\sum_{i=1}^{t-2} u_{i+1}^{2} K^{2}\left[\left(x_{t}-x_{i}\right) / h\right] .
$$

This, together with (6.8) and (8.11)-(8.12) in Lemma 8.2, implies that

$$
\begin{aligned}
E Z_{t}^{2} & \leq \frac{1}{4} E Z_{t 12}^{4}+\frac{A^{4}}{4} E\left(\sum_{i=1}^{t-2} K^{2}\left[\left(x_{t}-x_{i}\right) / h\right]\right)^{2} \\
& \leq C t^{3 / 2} h^{3}+C h^{2} \sum_{1 \leq i<j \leq t-2} \frac{1}{\sqrt{t-j}} \frac{1}{\sqrt{j-i}}+C h \sum_{i=1}^{t-2} \frac{1}{\sqrt{t-i}} \\
& \leq C_{1}\left(t^{3 / 2} h^{3}+h^{2} t+h \sqrt{t}\right) \leq C_{2} t^{3 / 2} h^{3},
\end{aligned}
$$

since $t \leq n$ and $n h^{2} \rightarrow \infty$. This proves (8.54).

To prove (8.55), by noting that, for $s<t$,

$$
\sum_{\substack{i, j=1 \\ i<j}}^{s-2} \sum_{\substack{k, l=1 \\ k<l}}^{t-2}=\sum_{\substack{i, j=1 \\ i<j}}^{s-2}\left(\sum_{\substack{k, l=1 \\ k<l}}^{s-2}+\sum_{l=s-1}^{s} \sum_{k=1}^{l-1}+\sum_{l=s+1}^{t-2} \sum_{k=1}^{l-1}\right)
$$

and

$$
4 \sum_{\substack{i, j=1 \\ i<j}}^{s-2} \sum_{\substack{k, l=1 \\ k<l}}^{s-2} \sum_{\substack{i, j=1 \\ i \neq j}}^{s-2} \sum_{\substack{k, l=1 \\ k \neq l}}^{s-2}=\sum_{i \neq j \neq k \neq l}^{s-2}+4 \sum_{\substack{i \neq j \neq k \\ l=i}}^{s-2}+2 \sum_{\substack{i \neq j \\ k=i, l=j}}^{s-2},
$$

we may write, with the notation $I(\ldots)$ as in Lemma 8.3,

$$
\begin{aligned}
Z_{s} Z_{t} & =\sum_{\substack{i, j=1 \\
i<j}}^{s-2} \sum_{\substack{k, l=1 \\
k<l}}^{t-2} I(i, j, k, l, s, t) \\
& =I_{1 s t}+I_{2 s t}+I_{3 s t}+I_{4 s t}+I_{5 s t},
\end{aligned}
$$


where, by symmetry and (8.17),

$$
\begin{aligned}
\left|E I_{1 s t}\right|= & \left|\sum_{i \neq j \neq k \neq l}^{s-2} E I(\ldots \ldots)\right| \\
\leq & \frac{C h^{4}}{t-s} \sum_{1 \leq i<j<k<l \leq s-2} \frac{1}{\sqrt{s-l}} \frac{1}{\sqrt{l-k}} \frac{1}{\sqrt{j-i}} \\
& +\frac{C h^{4}}{\sqrt{t-s}} \sum_{1 \leq i<j<k<l \leq s-2} \frac{1}{s-l} \frac{1}{\sqrt{l-k}} \frac{1}{\sqrt{j-i}} \\
\leq & C h^{4}\left(\frac{s^{5 / 2}}{t-s}+\frac{s^{2} \log s}{\sqrt{t-s}}\right) ;
\end{aligned}
$$

by $\left|u_{j}\right| \leq A$ and (8.15) with $l=i$ in Lemma 8.2,

$$
\begin{aligned}
& \left|E I_{2 s t}\right|=4\left|\sum_{\substack{i \neq j \neq k \\
l=i}}^{s-2} E I(\ldots . .)\right| \\
& \leq 4 A^{4} \sum_{i \neq j \neq k}^{s-2} E\left\{K\left[\left(x_{s}-x_{i}\right) / h\right] K\left[\left(x_{t}-x_{i}\right) / h\right] K\left[\left(x_{s}-x_{j}\right) / h\right] K\left[\left(x_{t}-x_{k}\right) / h\right]\right\} \\
& \leq \frac{C h^{4}}{\sqrt{t-s}} \sum_{1 \leq i<j<k \leq s-2} \frac{1}{\sqrt{s-k}} \frac{1}{\sqrt{j-i}} \\
& \leq \frac{C h^{4} s^{2}}{\sqrt{t-s}}
\end{aligned}
$$

by $\left|u_{j}\right| \leq A$ and (8.14) with $k=i$ and $l=j$ in Lemma 8.2,

$$
\begin{aligned}
& \left|E I_{3 s t}\right|=2\left|\sum_{\substack{i \neq j \\
k=i, l=j}}^{s-2} E I(\ldots . .)\right| \\
\leq & 4 A^{4} \sum_{i \neq j}^{s-2} E\left\{K\left[\left(x_{s}-x_{i}\right) / h\right] K\left[\left(x_{t}-x_{i}\right) / h\right] K\left[\left(x_{s}-x_{j}\right) / h\right] K\left[\left(x_{t}-x_{j}\right) / h\right]\right\} \\
\leq & \frac{C h^{3}}{\sqrt{t-s}} \sum_{1 \leq i<j \leq s-2} \frac{1}{\sqrt{s-j}} \\
\leq & \frac{C h^{3} s^{3 / 2}}{\sqrt{t-s}}
\end{aligned}
$$


by $(8.18)$ in Lemma 8.3 ,

$$
\begin{aligned}
& \left|E I_{5 s t}\right|=\sum_{\substack{i, j=1 \\
i<j}}^{s-2} \sum_{l=s+1}^{t-2}\left(\sum_{k=1}^{s-1}+\sum_{k=s}^{l-1}\right)|E I(\ldots \ldots)| \\
& \leq \sum_{\substack{i, j=1 \\
i<j}}^{s-2} \sum_{l=s+1}^{t-2} \sum_{k=1}^{s-1} \frac{C h^{4}}{t-l} \frac{1}{\sqrt{l-k}} \frac{1}{\sqrt{s-j}} \frac{1}{\sqrt{j-i}} \\
& +\sum_{\substack{i, j=1 \\
i<j}}^{s-2} \sum_{l=s+1}^{t-2} \sum_{k=s}^{l-1} \frac{C h^{4}}{t-l} \frac{1}{\sqrt{l-s}} \frac{1}{\sqrt{s-j}} \frac{1}{\sqrt{j-i}} \\
& \leq C h^{4} s\left(\sum_{l=s+1}^{t-2} \sum_{k=1}^{s-1} \frac{1}{t-l} \frac{1}{\sqrt{l-k}}+\sum_{l=s+1}^{t-2} \sum_{k=s}^{l-1} \frac{1}{t-l} \frac{1}{\sqrt{l-s}}\right) \\
& \leq C h^{4} s\left[\sqrt{t-s} \log (t-s)+\frac{s \log (t-s)}{\sqrt{t-s}}\right] \text {. }
\end{aligned}
$$

As for $I_{4 s t}$, by noting

$$
\begin{gathered}
\sum_{\substack{i, j=1 \\
i<j}}^{s-2} \sum_{l=s-1}^{s} \sum_{k=1}^{l-1}=\frac{1}{2} \sum_{\substack{i, j=1 \\
i \neq j}}^{s-2}\left(\sum_{l=s-1}^{s} \sum_{k=1}^{s-2}+\sum_{l=s-1}^{s} \sum_{k=s-1}^{l-1}\right) \\
=\frac{1}{2} \sum_{\substack{i, j, k=1 \\
i \neq j \neq k}}^{s-2} \sum_{l=s-1}^{s}+\sum_{\substack{i, j \\
i \neq j, k=i}}^{s-2} \sum_{l=s-1}^{s}+\frac{1}{2} \sum_{\substack{i, j=1 \\
i \neq j}}^{s-2} \sum_{l=s-1}^{s} \sum_{k=s-1}^{l-1},
\end{gathered}
$$

it follows from $\left|u_{j}\right| \leq A,(8.14)-(8.15)$ in Lemma 8.2,

$$
\begin{aligned}
& \left|E I_{4 s t}\right| \leq \sum_{\substack{i, j=1 \\
i<j}}^{s-2} \sum_{l=s-1}^{s} \sum_{k=1}^{l-1}|E I(\ldots \ldots)| \\
\leq & A^{4}\left(\frac{1}{2} \sum_{\substack{i, j, k=1 \\
i \neq j \neq k}}^{s-2} \sum_{\substack{l=s-1 \\
s}}^{s} \sum_{\substack{i, j \\
i \neq j, k=i}}^{s-2} \sum_{l=s-1}^{s}+\frac{1}{2} \sum_{\substack{i, j=1 \\
i \neq j}}^{s-2} \sum_{l=s-1}^{s} \sum_{k=s-1}^{l-1}\right) \chi_{s t}(i, j, k, l) \\
\leq & \frac{C h^{4}}{\sqrt{t-s}} \sum_{\substack{1 \leq i<j<k \leq s-2 \\
\sqrt{s-k}}} \frac{1}{\sqrt{j-i}} \\
& +\frac{C h^{3}}{\sqrt{t-s}} \sum_{\substack{1 \leq i<j \leq s-2 \\
\leq}} \frac{1}{\sqrt{s-j}} \\
\sqrt{t-s} &
\end{aligned}
$$

since $s \leq n$ and $n h^{2} \rightarrow \infty$.

By virtue of (8.57)-(8.62), the result (8.55) follows from a simple calculation. The proof of Proposition 6.4 is now complete. 
8.6 Proof of Proposition 6.5 . First consider (6.6). For $i=j-1$ or $j$, it follows from (8.8) with $p(x)=K\left(x-x_{i} / h\right) K\left(x-x_{j} / h\right)$ that

$$
\begin{aligned}
& \left|E\left\{u_{i+1} u_{j+1} K\left[\left(x_{t}-x_{i}\right) / h\right] K\left[\left(x_{t}-x_{j}\right) / h\right]\right\}\right| \\
\leq & E\left\{\left|u_{i+1}\right|\left|u_{j+1}\right| E\left(K\left[\left(x_{t}-x_{i}\right) / h\right] K\left[\left(x_{t}-x_{j}\right) / h\right] \mid \mathcal{F}_{j+1}\right)\right\} \\
\leq & \frac{C h}{\sqrt{t-j}} E\left(\left|u_{i+1}\right|\left|u_{j+1}\right|\right) .
\end{aligned}
$$

Similarly, for $i \leq j-2$, we have

$$
\begin{aligned}
E\left\{\left|u_{i+1}\right| K\left[x+\left(x_{j}-x_{i}\right) / h\right]\right\} & \leq E\left\{\left|u_{i+1}\right| E\left(K\left[x+\left(x_{j}-x_{i}\right) / h\right] \mid \mathcal{F}_{i+1}\right)\right\} \\
& \leq \frac{C h}{\sqrt{t-j}} E\left(\left|u_{i+1}\right|\right),
\end{aligned}
$$

uniformly for $x \in R$. The result (8.64), together with the usage of (8.7) with $p(x)=$ $K\left(x-x_{i} / h\right) K\left(x-x_{j} / h\right)$, yields that

$$
\begin{aligned}
& \left|E\left\{u_{i+1} u_{j+1} K\left[\left(x_{t}-x_{i}\right) / h\right] K\left[\left(x_{t}-x_{j}\right) / h\right]\right\}\right| \\
\leq & E\left\{\left|u_{i+1}\right|\left|E\left(u_{j+1} K\left[\left(x_{t}-x_{i}\right) / h\right] K\left[\left(x_{t}-x_{j}\right) / h\right] \mid \mathcal{F}_{j}\right)\right|\right\} \\
\leq & \frac{C h}{t-j} \int_{-\infty}^{\infty} E\left\{\left|u_{i+1}\right| K\left[x+\left(x_{j}-x_{i}\right) / h\right]\right\} K(x) d x \\
\leq & \frac{C h^{2}}{t-j} \frac{1}{\sqrt{j-i}} E\left|u_{i+1}\right| .
\end{aligned}
$$

Combining (8.63) and (8.65), we obtain that, for $1 \leq k \leq t-r$ and $r \geq 2$,

$$
\begin{aligned}
E Z_{t k r}^{2} & =2\left(\sum_{\substack{i, j=k \\
j-i \geq 2}}^{t-r}+\sum_{\substack{i, j=k \\
0 \leq j-i \leq 1}}^{t-r} E\left\{u_{i+1} u_{j+1} K\left[\left(x_{t}-x_{i}\right) / h\right] K\left[\left(x_{t}-x_{j}\right) / h\right]\right\}\right. \\
& \leq C \max _{1 \leq i, j \leq n} E\left[\left|u_{i}\right|\left(1+\left|u_{j}\right|\right)\right]\left(\sum_{\substack{i, j=k \\
j-i \geq 2}}^{t-r} \frac{h^{2}}{t-j} \frac{1}{\sqrt{j-i}}+\sum_{\substack{i, j=k \\
0 \leq j-i \leq 1}}^{t-r} \frac{h}{\sqrt{t-j}}\right) \\
& \leq C \max _{1 \leq i, j \leq n} E\left[\left|u_{i}\right|\left(1+\left|u_{j}\right|\right)\right](h \log t+1) h \sqrt{t-r-k},
\end{aligned}
$$

which yields (6.6), since $h \log ^{2} n \rightarrow 0$.

The proof of (6.7) is similar. We omit the details.

We next prove (6.8). Recalling $\sup _{x} K(x)<\infty,\left|u_{j}\right| \leq A$ and the notation defined in 
Lemma 8.3, we have

$$
\begin{aligned}
E Z_{412}^{4} \leq & \sum_{\substack{i, j, k, l=1 \\
i \neq j \neq k \neq l}}^{t-2}|I(i, j, k, l, t, t)|+C \sum_{\substack{i, j, k=1 \\
i \neq j \neq k}}^{t-2} E\left\{K\left[\left(x_{t}-x_{i}\right) / h\right] K\left[\left(x_{t}-x_{j}\right) / h\right] K\left[\left(x_{t}-x_{k}\right) / h\right]\right\} \\
& +C \sum_{\substack{i, j=1 \\
i \neq j}}^{t-2} E\left\{K\left[\left(x_{t}-x_{i}\right) / h\right] K\left[\left(x_{t}-x_{j}\right) / h\right]\right\}+C \sum_{i=1}^{t-2} E K\left[\left(x_{t}-x_{i}\right) / h\right] \\
\leq & \tilde{Z}_{1 t}+\tilde{Z}_{2 t}+\tilde{Z}_{3 t}+\tilde{Z}_{4 t} .
\end{aligned}
$$

Using (8.19), we have

$$
\begin{aligned}
\tilde{Z}_{1 t} & \leq C h^{4} \sum_{l=4}^{t-2} \sum_{k=3}^{l-1} \sum_{j=2}^{k-1} \sum_{i=1}^{j-1} \frac{1}{t-l} \frac{1}{\sqrt{l-k}} \frac{1}{\sqrt{k-j}} \frac{1}{\sqrt{j-i}} \\
& \leq C h^{4} t^{3 / 2} \log t
\end{aligned}
$$

Using (8.11)-(8.13) with $p_{1}(x)=p_{2}(x)=p_{3}(x)=K(x)$, we obtain

$$
\begin{aligned}
\tilde{Z}_{2 t}+\tilde{Z}_{3 t}+\tilde{Z}_{4 t} \leq & \sum_{k=3}^{t-2} \sum_{j=2}^{k-1} \sum_{i=1}^{j-1} \frac{C h^{3}}{\sqrt{t-k}} \frac{1}{\sqrt{k-j}} \frac{1}{\sqrt{j-i}} \\
& +\sum_{j=2}^{t-2} \sum_{i=1}^{j-1} \frac{C h^{2}}{\sqrt{t-j}} \frac{1}{\sqrt{j-i}}+\sum_{j=1}^{t-2} \frac{C h}{\sqrt{t-j}} \\
\leq & C h^{3} t^{3 / 2}+C h^{2} t+C h \sqrt{t} \leq C h^{3} t^{3 / 2}
\end{aligned}
$$

since $t \leq n$ and $n h^{2} \rightarrow \infty$. By virtue of (8.66)-(8.68), we obtain (6.8).

Finally for (6.9). For $i<j$, write

$$
I I_{i, j}=E\left\{u_{i+1} u_{j+1} E\left(K\left[\left(x_{t}-x_{i}\right) / h\right] \mid \mathcal{F}_{t-m}\right) E\left(K\left[\left(x_{t}-x_{j}\right) / h\right] \mid \mathcal{F}_{t-m}\right)\right\} .
$$

Using (8.6), (8.8) and $\left|u_{j}\right| \leq A$, simple calculations show that

$$
\begin{gathered}
I I_{i, j}=\frac{h}{\sqrt{m}} \int_{-\infty}^{\infty} E\left\{u_{i+1} u_{j+1} E\left(K\left[\left(x_{t}-x_{i}\right) / h\right] \mid \mathcal{F}_{t-m}\right)\right. \\
\left.K\left[\left(\rho^{m} x_{t-m}-x_{j}\right) / h+x\right]\right\} h_{t-m, t}\left(\frac{h x}{\sqrt{m}}\right) d x+\tilde{Z}_{5 n} \\
=\frac{h^{2}}{m} \int_{-\infty}^{\infty} \int_{-\infty}^{\infty} E\left\{u_{i+1} u_{j+1} K\left[\left(\rho^{m} x_{t-m}-x_{i}\right) / h+y\right] K\left[\left(\rho^{m} x_{t-m}-x_{j}\right) / h+x\right]\right\} \\
h_{t-m, t}\left(\frac{h x}{\sqrt{m}}\right) h_{t-m, t}\left(\frac{h y}{\sqrt{m}}\right) d x d y+\tilde{Z}_{5 n}+\tilde{Z}_{6 n},
\end{gathered}
$$


where $h_{s, t}(z)$ is the density given in (8.2),

$$
\begin{aligned}
\left|\tilde{Z}_{5 n}\right| & \leq \frac{C h}{m} E\left\{\left(1+\sum_{k=0}^{\infty}(k+1)^{-1-\delta}\left|\epsilon_{t-m-k}\right|\right)\left|E\left(K\left[\left(x_{t}-x_{i}\right) / h\right] \mid \mathcal{F}_{t-m}\right)\right|\right\} \\
& \leq \frac{C h^{2}}{m^{3 / 2}}, \\
\left|\tilde{Z}_{6 n}\right| & \leq \frac{C h^{2}}{m^{3 / 2}} \int_{-\infty}^{\infty} E\left\{\left(1+\sum_{k=0}^{\infty}(k+1)^{-1-\delta}\left|\epsilon_{t-m-k}\right|\right) K\left[\left(\rho^{m} x_{t-m}-x_{j}\right) / h+x\right]\right\} d x \\
& \leq \frac{C h^{2}}{m^{3 / 2}} .
\end{aligned}
$$

On the other hand, using (8.7) with $p(z)=K\left[\left(\rho^{m} z-x_{i} / h+y\right] K\left[\left(\rho^{m} z-x_{j} / h+x\right]\right.\right.$,

$$
\begin{aligned}
& \left|E\left\{u_{j+1} K\left[\left(\rho^{m} x_{t-m}-x_{i}\right) / h+y\right] K\left[\left(\rho^{m} x_{t-m}-x_{j}\right) / h+x\right] \mid \mathcal{F}_{j}\right\}\right| \\
\leq & \frac{C h}{t-m-j} \int_{-\infty}^{\infty} K\left[( \rho ^ { m } z - x _ { i } / h + y ] K \left[\left(\rho^{m} z-x_{j} / h+x\right] d z\right.\right. \\
\leq & \frac{C h}{t-j} \int_{-\infty}^{\infty} K\left(\rho^{m} z\right) K\left[\rho^{m} z+\left(x_{j}-x_{i}\right) / h+y-x\right] d z .
\end{aligned}
$$

Taking these estimates into (8.69) and recalling $h_{s, t}(z) \leq C_{0}$ and $\int_{-\infty}^{\infty} h_{s, t}(z) d z=1$, we obtain

$$
\begin{aligned}
I I_{i, j} & \leq \frac{C_{1} h^{3}}{m(t-j)} E\left\{\int_{-\infty}^{\infty} \int_{-\infty}^{\infty} \int_{-\infty}^{\infty} K\left(\rho^{m} z\right) K\left[\rho^{m} z+\left(x_{j}-x_{i}\right) / h+y-x\right]\right. \\
& \leq \frac{C_{3} h^{2}}{\sqrt{m}(t-j)}+\frac{C h^{2}}{m^{3 / 2}}
\end{aligned}
$$

This, together with (8.8), yields that

$$
\begin{aligned}
& E\left|\sum_{i=1}^{t-m-1} u_{i+1} E\left(K\left[\left(x_{t}-x_{i}\right) / h\right] \mid \mathcal{F}_{t-m}\right)\right|^{2} \\
\leq & 2 \sum_{1 \leq i<j \leq t-m-1} I I_{i, j}+A^{2} \sum_{i=1}^{t-m-1} E\left|E\left(K\left[\left(x_{t}-x_{i}\right) / h\right] \mid \mathcal{F}_{t-m}\right)\right|^{2} \\
\leq & 2 \sum_{1 \leq i<j \leq t-m-1}\left(\frac{C_{3} h^{2}}{\sqrt{m}(t-j)}+\frac{C h^{2}}{m^{3 / 2}}\right)+C \sum_{i=1}^{t-m-1} \frac{h^{2}}{m} \\
\leq & \frac{C h^{2} t^{2}}{m^{3 / 2}}+\frac{C h^{2} t \log (t-m)}{\sqrt{m}}+\frac{C h^{2} t}{m} .
\end{aligned}
$$

This proves (6.9) and also completes the proof of Proposition 6.5. 
8.7 Proof of Proposition 6.6. Write $\tau_{n, t}^{*}=\tau_{n, t}-\tau_{n, t-1}$ and recall $E\left(\tau_{n, t}^{*} \mid \mathcal{F}_{n, t-1}^{0}\right)=$ $\sigma^{-2} E\left[u_{t+1}^{2} \mid \mathcal{F}_{t}\right]$ by $(6.35)$. We have, for $0 \leq r \leq T$,

$$
\begin{aligned}
{\left[M_{n}, V\right]_{r}=} & \frac{1}{n} \sum_{t=1}^{[n r] \wedge n} Y_{n t}^{*}+\frac{1}{n \sigma^{2}} \sum_{t=1}^{[n r] \wedge n} Y_{n t}^{*}\left[\tau_{n, t}^{*}-E\left(\tau_{n, t}^{*} \mid \mathcal{F}_{n, t-1}^{0}\right)\right] \\
& +\frac{1}{n \sigma^{2}} \sum_{t=1}^{[n r] \wedge n} Y_{n t}^{*}\left[E\left(u_{t+1}^{2} \mid \mathcal{F}_{t}\right)-\sigma^{2}\right]+R_{n}(r) \\
:= & Z_{1 n}(r)+Z_{2 n}(r)+Z_{3 n}(r)+R_{n}(r),
\end{aligned}
$$

where, by recalling $\tau_{n, j-1}<n r \leq \tau_{n, j}$ and (6.34),

$$
\begin{aligned}
\sup _{0 \leq r \leq T}\left|R_{n}(r)\right| & \leq \sup _{0 \leq r \leq 1}\left|R_{n}(r)\right|+\sup _{1 \leq r \leq T}\left|R_{n}(r)\right| \\
& \leq n^{-1} \max _{1 \leq k \leq n}\left[|[n r]-(j-1)|\left|Y_{n k}^{*}\right| \tau_{n, k}^{*}\right]+\left(T+\left|1-\tau_{n, n} / n\right|\right) / \sqrt{n} \\
& \leq C n^{\delta-1} \max _{1 \leq k \leq n}\left[\left|Y_{n k}^{*}\right| \tau_{n, k}^{*}\right]+C / \sqrt{n}
\end{aligned}
$$

for any $\delta>1 / 2$. Since $\left|u_{k}\right| \leq C$, it follows from (6.35), (6.8) and

$$
Y_{n t}^{*}=_{d} \frac{\sqrt{n}}{d_{n}} Z_{t 11}
$$

(where $Z_{t k r}=\sum_{i=k}^{t-r} u_{i+1} K\left[\left(x_{t}-x_{i}\right) / h\right]$ is defined as in Proposition 6.5) that

$$
\begin{aligned}
E \max _{1 \leq k \leq n}\left[\left|Y_{n k}^{*}\right| \tau_{n, k}^{*}\right] & \leq\left\{\sum_{k=1}^{n} E\left[\left|Y_{n k}^{*}\right|^{4} E\left(\tau_{n, k}^{* 4} \mid \mathcal{F}_{n, t-1}^{0}\right)\right]\right\}^{1 / 4} \\
& \leq C\left(n h^{2}\right)^{-1 / 4}\left(E Z_{t 11}^{4}\right)^{1 / 4} \leq C\left(n h^{2}\right)^{-1 / 4}\left(C+E Z_{t 12}^{4}\right)^{1 / 4} \\
& \leq C\left(n h^{2}\right)^{-1 / 4}\left(1+h^{4} \sum_{t=2}^{n} t^{3 / 2} \log t+h^{3} \sum_{t=1}^{n} t^{3 / 2}\right)^{1 / 4} \\
& \leq C n^{3 / 8} h^{1 / 4},
\end{aligned}
$$

whenever $n h^{2} \rightarrow \infty$ and $h \log ^{2} n \rightarrow 0$. Taking this estimate into (8.71), we obtain $\sup _{0 \leq r \leq T}\left|R_{n}(r)\right|=o_{P}(1)$ by choosing $\delta<1 / 8$. Note that $\left\{Y_{n t}^{*}\left[\tau_{n, t}^{*}-E\left(\tau_{n, t}^{*} \mid \mathcal{F}_{n, t-1}^{0}\right)\right], \mathcal{F}_{n, t}^{0}\right\}_{1 \leq t \leq n}$ forms a martingale difference. It follows from the maximal inequality for martingales, (6.35) and (6.6) that

$$
\begin{aligned}
E \sup _{0 \leq r \leq T}\left|Z_{2 n}(r)\right|^{2} & \leq \frac{C}{n^{2}} \sum_{t=1}^{n} E\left\{Y_{n t}^{* 2} E\left(\tau_{n, t}^{* 2} \mid \mathcal{F}_{n, t-1}^{0}\right)\right\} \\
& \leq \frac{C_{1}}{n d_{n}^{2}} \sum_{t=1}^{n} E\left(Z_{t 11}\right)^{2} \leq \frac{C_{2}}{n^{5 / 2} h} \sum_{t=1}^{n}(1+h \sqrt{t}) \leq C / n,
\end{aligned}
$$


which yields that $\sup _{0 \leq r \leq T}\left|Z_{2 n}(r)\right|=o_{P}(1)$. To estimate $Z_{3 n}(r)$, recall

$$
E\left|Y_{n t}^{*}\right| \leq \frac{C}{n^{1 / 4} \sqrt{h}}\left(E\left|Z_{t 11}\right|^{2}\right)^{1 / 2} \leq C_{1}(t / n)^{1 / 4} .
$$

It is readily seen that $\sum_{t=1}^{n} E\left|Y_{n t}^{*}\right| \leq C n$ and for any $c_{n} / n \rightarrow 0, \sum_{t=1}^{c_{n}} E\left|Y_{n t}^{*}\right| \leq C c_{n}^{5 / 4} n^{-1 / 4}=$ $o(n)$. By virtue of these facts and $E\left[u_{t+1}^{2} \mid \mathcal{F}_{t}\right] \rightarrow_{a . s .} \sigma^{2}$, it follows from Lemma 8.6 that

$$
E \sup _{0 \leq r \leq T}\left|Z_{3 n}(r)\right|^{1 / 2} \leq \frac{C}{\sqrt{n}} E\left(\sum_{t=1}^{n}\left|Y_{n t}^{*}\right|\left|\left[E\left(u_{t+1}^{2} \mid \mathcal{F}_{t}\right)-\sigma^{2}\right]\right|\right)^{1 / 2}=o(1),
$$

which yields that $\sup _{0 \leq r \leq T}\left|Z_{3 n}(r)\right|=o_{P}(1)$.

Hence, it suffices to show that

$$
\sup _{0 \leq r \leq T}\left|Z_{1 n}(r)\right|={ }_{d} \frac{1}{\sqrt{n} d_{n}} \max _{1 \leq k \leq n}\left|\sum_{t=1}^{k} \sum_{i=1}^{t-1} u_{i+1} K\left[\left(x_{t}-x_{i}\right) / h\right]\right|=o_{P}(1) .
$$

Note that, with $\eta_{n}=\epsilon_{n} n$ and $m=\eta_{n} / 2$ where $\epsilon_{n}$ is chosen such that $\epsilon_{n} \rightarrow 0$ and $\epsilon_{n}^{-1 / 2} h \log ^{2} n \rightarrow 0$,

$$
\begin{aligned}
& \max _{1 \leq k \leq n}\left|\sum_{t=1}^{k} \sum_{i=1}^{t-1} u_{i+1} K\left[\left(x_{t}-x_{i}\right) / h\right]\right| \leq C n+\max _{1 \leq k \leq n}\left|\sum_{t=1}^{k} Z_{t 12}\right| \\
& \leq C n+2 \sum_{t=1}^{\eta_{n}}\left|Z_{t 12}\right|+\sum_{\eta_{n}+1 \leq t \leq n}\left|\sum_{i=t-m+1}^{t-2} u_{i+1} K\left[\left(x_{t}-x_{i}\right) / h\right]\right| \\
& +\max _{\eta_{n}+1 \leq k \leq n}\left|\sum_{t=\eta_{n}+1}^{k} \sum_{i=1}^{t-m} u_{i+1} K\left[\left(x_{t}-x_{i}\right) / h\right]\right| \\
& :=C n+2 H_{1 n}+H_{2 n}+H_{3 n} .
\end{aligned}
$$

By (6.6), it follows that

$$
\begin{aligned}
E H_{1 n} & \leq \sum_{t=1}^{\eta_{n}}\left(E\left|Z_{t 12}\right|^{2}\right)^{1 / 2} \leq C h^{1 / 2} \sum_{t=1}^{\eta_{n}} t^{1 / 4} \leq C_{1} \epsilon_{n} n^{5 / 4} h^{1 / 2}, \\
E H_{2 n} & \leq \sum_{\eta_{n}+1 \leq t \leq n}\left(E\left|Z_{t(m-1) 2}\right|^{2}\right)^{1 / 2} \\
& \leq C n m^{1 / 4} \sqrt{h} \leq C_{1} \epsilon_{n}^{1 / 4} n^{5 / 4} h^{1 / 2} .
\end{aligned}
$$

By virtue of (8.76) and (8.77), we have

$$
C n+2 H_{1 n}+H_{2 n} \leq C n^{5 / 4} h^{1 / 2}\left[1 /\left(n h^{2}\right)^{1 / 4}+O_{P}\left(\epsilon_{n}^{1 / 4}\right)\right]=o_{P}\left(n^{5 / 4} h^{1 / 2}\right) .
$$

Taking this fact into (8.75), the result (8.74) will follow if we prove

$$
H_{3 n}=\max _{\eta_{n}+1 \leq k \leq n}\left|\sum_{t=\eta_{n}+1}^{k} Z_{t 1 m}\right|=o_{P}\left(n^{5 / 4} \sqrt{h}\right) .
$$


It is readily seen that

$$
\begin{aligned}
H_{3 n} & \leq \max _{\eta_{n}+1 \leq k \leq n}\left|\sum_{t=\eta_{n}+1}^{k}\left\{Z_{t 1 m}-E\left(Z_{t 1 m} \mid \mathcal{F}_{t-m}\right)\right\}\right|+\sum_{t=\eta_{n}+1}^{n}\left|E\left(Z_{t 1 m} \mid \mathcal{F}_{t-m}\right)\right| \\
& :=H_{3 n 1}+H_{3 n 2} .
\end{aligned}
$$

Note that, for fixed $m,\left\{Z_{t 1 m}-E\left(Z_{t 1 m} \mid \mathcal{F}_{t-m}, \mathcal{F}_{t-m}\right\}_{t \geq 1}\right.$ forms a martingale difference. It follows that

$$
E H_{3 n 1}^{2} \leq C \sum_{t=1}^{n} E Z_{t 1 m}^{2} \leq C h \sum_{t=1}^{n} t^{1 / 2} \leq C_{1} h n^{3 / 2}
$$

which yields that $H_{3 n 1}=o_{P}\left(n^{5 / 4} \sqrt{h}\right)$.

As for $H_{3 n 2}$, by noting that $u_{i+1}$ is $\mathcal{F}_{t-m}$ measurable when $i \leq t-m-1$, it follows from (6.9) that

$$
\begin{aligned}
E H_{3 n 2} & \leq C n+\sum_{t=m+1}^{n} E\left|\sum_{i=1}^{t-m-1} u_{i+1} E\left(K\left[\left(x_{t}-x_{i}\right) / h\right] \mid \mathcal{F}_{t-m}\right)\right| \\
& \leq C n+\sum_{t=m+1}^{n}\left(E\left|\sum_{i=1}^{t-m-1} u_{i+1} E\left(K\left[\left(x_{t}-x_{i}\right) / h\right] \mid \mathcal{F}_{t-m}\right)\right|^{2}\right)^{1 / 2} \\
& \leq C n+C \sum_{t=m+1}^{n}\left(\frac{h^{2} t^{2}}{m^{3 / 2}}+\frac{h^{2} t \log (t-m)}{\sqrt{m}}+\frac{h^{2} t}{m}\right)^{1 / 2} \\
& \leq C n+C h\left(\frac{n^{2}}{m^{3 / 4}}+\frac{n^{3 / 2} \log n}{m^{1 / 4}}+\frac{n^{3 / 2}}{m^{1 / 2}}\right) \\
& \leq C n+C \epsilon_{n}^{-1 / 4} n^{5 / 4} h \log n \\
& =o_{P}\left(n^{5 / 4} \sqrt{h}\right)
\end{aligned}
$$

since $\epsilon_{n}^{-1 / 4} \sqrt{h} \log n=o(1)$. This proves (8.78), and hence the proof of Proposition 6.6 is complete.

8.8 Proof of Proposition 6.7. As in the proof of Proposition 6.6, write $\tau_{n, t}^{*}=$ $\tau_{n, t}-\tau_{n, t-1}$. Recall $E\left(\tau_{n, t}^{*} \mid \mathcal{F}_{n, t-1}^{0}\right)=\sigma^{-2} E\left[u_{t+1}^{2} \mid \mathcal{F}_{t}\right]$ by (6.35). We have,

$$
\begin{aligned}
& {\left[M_{n}\right]_{1}=} \frac{1}{n} \sum_{t=1}^{n} Y_{n t}^{* 2}+\frac{1}{n \sigma^{2}} \sum_{t=1}^{n} Y_{n t}^{* 2}\left[\tau_{n, t}^{*}-E\left(\tau_{n, t}^{*} \mid \mathcal{F}_{n, t-1}^{0}\right)\right] \\
& \frac{1}{n \sigma^{2}} \sum_{t=1}^{n} Y_{n t}^{* 2}\left[E\left(u_{t+1}^{2} \mid \mathcal{F}_{t}\right)-\sigma^{2}\right]+R_{n j}^{*} \\
&:=\frac{1}{n} \sum_{t=1}^{n} Y_{n t}^{* 2}+L_{1 n}+L_{2 n}+R_{n j}^{*}
\end{aligned}
$$


where, for some $1 \leq j \leq n$ satisfying $\tau_{n, j-1}<n \leq \tau_{n, j}$,

$$
\left|R_{n j}^{*}\right| \leq \frac{1}{n}\left(1-\frac{\tau_{n, n}}{n}\right)+\frac{1}{n} \sum_{t=j}^{n} Y_{n t}^{* 2} \tau_{n, t}^{*} .
$$

Note that $j$ is a random variable satisfying

$$
n-j \leq \max _{1 \leq j \leq n}\left|\tau_{n, j}-j\right|+1 / n=o\left(n^{\delta}\right), \quad \text { a.s. }
$$

for any $\delta>0$, due to (6.34). It is readily seen that

$$
\left|R_{n j}^{*}\right| \leq o_{P}(1)+\frac{1}{n} \sum_{t=n-n^{\delta}}^{n} Y_{n t}^{* 2} \tau_{n t}^{*}=o_{P}(1),
$$

where we have used the fact that, for any $\delta>0$,

$$
\begin{aligned}
\frac{1}{n} \sum_{t=n-n^{\delta}}^{n} E\left[Y_{n t}^{* 2} \tau_{n t}^{*}\right] & \leq \frac{1}{n} \sum_{t=n-n^{\delta}}^{n} E\left(\left|Y_{t n}^{* 2}\right| E\left[\left(\tau_{n, t}-\tau_{n, t-1}\right)^{2} \mid \mathcal{F}_{n, t-1}^{0}\right]\right) \\
& \leq \frac{C}{n^{3 / 2} h} \sum_{t=n-n^{\delta}}^{n} E L_{t 11}^{2} \leq \frac{C}{n^{3 / 2}} \sum_{t=n-n^{\delta}}^{n}(1+h \log t) \sqrt{t} \\
& \leq \frac{C}{n^{3 / 2}}\left[n^{3 / 2}-\left(n-n^{\delta}\right)^{3 / 2}\right]=o(1)
\end{aligned}
$$

due to (6.34), (8.72), (6.6) and $h \log ^{2} n \rightarrow 0$.

By noting that $\left\{Y_{n t}^{* 2}\left[\tau_{n, t}^{*}-E\left(\tau_{n, t}^{*} \mid \mathcal{F}_{n, t-1}^{0}\right)\right], \mathcal{F}_{n, t-1}^{0}\right\}_{t \geq 1}$ forms a martingale difference, it follows from (6.35) and (6.8) that

$$
\begin{aligned}
E L_{1 n}^{2} & \leq \frac{C}{n^{2}} \sum_{t=1}^{n} E\left\{Y_{n t}^{* 4} E\left(\tau_{n, t}^{* 2} \mid \mathcal{F}_{n, t-1}^{0}\right)\right\} \\
& \leq \frac{C}{n d_{n}^{2}} \sum_{t=1}^{n} E\left|\sum_{i=1}^{n} u_{i+1} K\left(\frac{x_{t}-x_{i}}{h}\right)\right|^{4} \\
& \leq \frac{C}{n^{5 / 2} h} \sum_{t=1}^{n}\left(C+h^{4} t^{3 / 2} \log t+h^{3} t^{3 / 2}\right) \\
& \leq C h^{2}(1+h \log n)=o(1),
\end{aligned}
$$

since $h \log ^{2} n \rightarrow 0$, which implies that $L_{1 n}=o_{P}(1)$. To estimate $L_{2 n}$, recall that, by (6.6),

$$
E Y_{n t}^{* 2} \leq \frac{C n}{d_{n}^{2}} E\left|\sum_{i=1}^{t-1} u_{i+1} K\left(\frac{x_{t}-x_{i}}{h}\right)\right|^{2} \leq C(t / n)^{1 / 2}
$$

It is readily seen that $\sum_{t=1}^{n} E Y_{n t}^{* 2} \leq C n$ and for any $c_{n} / n \rightarrow 0, \sum_{t=1}^{c_{n}} E Y_{n t}^{* 2} \leq C c_{n}^{3 / 2} n^{-1 / 2}=$ $o(n)$. By virtue of these facts and $E\left[u_{t+1}^{2} \mid \mathcal{F}_{t}\right] \rightarrow_{a . s .} \sigma^{2}$, it follows from Lemma 8.6 that

$$
E\left|L_{2 n}\right|^{1 / 2} \leq \frac{C}{\sqrt{n}} E\left(\sum_{t=1}^{n} Y_{n t}^{* 2}\left|\left[E\left(u_{t+1}^{2} \mid \mathcal{F}_{t}\right)-\sigma^{2}\right]\right|\right)^{1 / 2}=o(1)
$$


which yields that $L_{2 n}=o_{P}(1)$.

By virtue of all these facts, namely $L_{1 n}+L_{2 n}+R_{n j}^{*}=o_{P}(1)$, and (8.80), we obtain (6.47). We next prove (6.46), which will follow if we prove that, under the extended probability space,

$$
\frac{1}{n} \sum_{t=1}^{n} Y_{n t}^{* 2} \rightarrow_{P} \eta^{2}
$$

In fact, by recalling (6.32)-(6.33), simple calculations show that

$$
\frac{1}{n} \sum_{t=1}^{n} Y_{n t}^{* 2}=\frac{\sigma^{2}}{d_{n}^{2}} \sum_{t=1}^{[n r]} \sum_{i=1}^{t-1} K^{2}\left\{c_{n}\left[G_{n}^{0}(t / n)-G_{n}^{0}(i / n)\right]\right\}+R_{1 n}^{*}+R_{2 n}^{*}
$$

where

$$
\begin{aligned}
& \left.R_{1 n}^{*}={ }_{d} \frac{1}{d_{n}^{2}} \sum_{t=1}^{n} \sum_{i=1}^{t-1}\left(u_{i+1}^{2}-\sigma^{2}\right) K^{2}\left[\left(x_{t}-x_{i}\right) / h\right]\right\}, \\
& R_{2 n}^{*}={ }_{d} \frac{2}{d_{n}^{2}} \sum_{t=1}^{n} \sum_{\substack{i, j=1 \\
i \neq j}}^{t-1} u_{i+1} u_{j+1} K\left[\left(x_{t}-x_{i}\right) / h\right] K\left[\left(x_{t}-x_{j}\right) / h\right] .
\end{aligned}
$$

The result (8.84) hence follows from Theorem 5.1, Propositions 6.3 and 6.4.

8.9 Proof of Proposition 6.8. As in (8.80), we have

$$
M_{n}(1)=\sum_{t=2}^{n}\left[V\left(\tau_{n, t} / n\right)-V\left(\tau_{n, t-1} / n\right)\right] Y_{n, t}^{*}+R_{n j}^{*}
$$

where, for some $1 \leq j \leq n$ satisfying $\tau_{n, j-1}<n \leq \tau_{n, j}$,

$$
\begin{aligned}
\left|R_{n j}^{*}\right| \leq & \frac{1}{\sqrt{n}}\left|V(1)-V\left(\tau_{n, n} / n\right)\right|+\left|Y_{n, j}^{*}\right|\left|V(1)-V\left(\tau_{n, j-1} / n\right)\right| \\
& +\left|\sum_{t=j}^{n}\left[V\left(\tau_{n, t} / n\right)-V\left(\tau_{n, t-1} / n\right)\right] Y_{n, t}^{*}\right| \\
:= & L_{3 n}+L_{4 n}+L_{5 n} .
\end{aligned}
$$

Write $\Omega_{j}=\left\{j: n-j \leq n^{\delta}\right.$, where $\left.\tau_{n, j-1}<n \leq \tau_{n, j}\right\}$. It follows from (6.34) that

$$
P\left(j \notin \Omega_{j}\right) \rightarrow 0
$$

This yields that, for any $\epsilon>0$,

$$
\begin{aligned}
P\left(L_{5 n} \geq \epsilon\right) & \leq P\left(j \notin \Omega_{j}\right)+P\left(j \in \Omega_{j}, L_{5 n} \geq \epsilon\right) \\
& \leq o(1)+\epsilon^{-2} E\left(I_{\left(j \in \Omega_{j}\right)} L_{5 n}^{2}\right) .
\end{aligned}
$$


Note that $\Omega_{j}$ is $\mathcal{F}_{n, t-1}^{0}$-measurable for $t \geq j$ and

$$
E\left\{\left[V\left(\tau_{n, t} / n\right)-V\left(\tau_{n, t-1} / n\right)\right] \mid \mathcal{F}_{n, t-1}\right\}=0 .
$$

It follows from the conditional arguments and (8.83) that

$$
\begin{aligned}
E\left(I_{\left(j \in \Omega_{j}\right)} L_{5 n}^{2}\right) & \leq E\left(I_{\left(j \in \Omega_{j}\right)} \sum_{t=j}^{n}\left[V\left(\tau_{n, t} / n\right)-V\left(\tau_{n, t-1} / n\right)\right]^{2} Y_{n, t}^{* 2}\right) \\
& \leq \sum_{t=n-n^{\delta}}^{n} E\left(\left[V\left(\tau_{n, t} / n\right)-V\left(\tau_{n, t-1} / n\right)\right]^{2} Y_{n, t}^{* 2}\right) \\
& \leq \sum_{t=n-n^{\delta}}^{n} E\left(Y_{n, t}^{* 2} E\left[\left[V\left(\tau_{n, t} / n\right)-V\left(\tau_{n, t-1} / n\right)\right]^{2} \mid \mathcal{F}_{n, t-1}^{0}\right]\right) \\
& \leq \frac{1}{n} \sum_{t=n-n^{\delta}}^{n} E\left(Y_{n, t}^{* 2}\left[\tau_{n, t}-\tau_{n, t-1}\right]\right) \rightarrow 0 .
\end{aligned}
$$

This yields that $L_{5 n}=o(1)$. By virtue of (6.34), $L_{3 n}=o_{p}(1)$ is obvious. As for $L_{4 n}$, similar to the proof of (8.88), we have

$$
\begin{aligned}
P\left(L_{4 n} \geq \epsilon\right) & \leq P\left(j \notin \Omega_{j}\right)+P\left(j \in \Omega_{j}, L_{4 n} \geq \epsilon\right) \\
& \leq o(1)+\epsilon^{-2} E\left(I_{\left(j \in \Omega_{j}\right)}^{2} L_{4 n}^{2}\right) \\
& \leq o(1)+\sum_{t=n-n^{\delta}}^{n} E\left(Y_{n, t}^{* 2} \sup _{\tau_{n, t-1}<n \leq \tau_{n, t}}\left[V(1)-V\left(\tau_{n, t-1} / n\right)\right]^{2}\right) \\
& \leq o(1)+\sum_{t=n-n^{\delta}}^{n} E\left\{Y_{n, t}^{* 2} E\left(\sup _{\tau_{n, t-1}<n \leq \tau_{n, t}}\left[V(1)-V\left(\tau_{n, t-1} / n\right)\right]^{2} \mid \mathcal{F}_{n, t-1}^{0}\right)\right\} \\
& \leq o(1)+\frac{1}{n} \sum_{t=n-n^{\delta}}^{n} E\left(Y_{n, t}^{* 2}\left[\tau_{n, t}-\tau_{n, t-1}\right]\right) \rightarrow 0 .
\end{aligned}
$$

Taking all these estimates into (8.87), we obtain the required (6.48).

\section{Supplement to the Simulations in Section 4}

The results given here supplement those reported in the published paper by the inclusion of additional parameter configurations. We refer readers to Section 4 of the published paper for discussion.

Simulations were conducted to evaluate the finite sample performance of the statistic $Z_{n}=S_{n} / \sqrt{2} V_{n}$ under the null and some local alternatives under various assumptions about the generating mechanism. The model followed (2.1) with $y_{t+1}=f\left(x_{t}\right)+u_{t+1}, x_{t}=$ 
$x_{t-1}+\eta_{t}, x_{0}=0$, and $\eta_{t}$ generated by an AR(1) process $\eta_{t}=\lambda \eta_{t-1}+\varepsilon_{t}$ or an MA(1) process $\eta_{t}=\varepsilon_{t}+\lambda \varepsilon_{t-1}$ with $\left(u_{t}, \varepsilon_{t}\right) \sim \operatorname{iidN}\left(0,\left(\begin{array}{ll}1 & r \\ r & 1\end{array}\right)\right)$. A linear null hypothesis $H_{0}: f(x)=$ $\theta_{0}+\theta_{1} x$ was used together with polynomial local alternatives $H_{1}: f(x)=\theta_{0}+\theta_{1} x+\rho_{n}|x|^{\nu}$, with $\rho_{n}=1 / n^{\frac{1}{4}+\frac{\nu}{3}} h^{\frac{1}{4}}$. The parameter settings were $\theta_{0}=0, \theta_{1}=1, \nu \in\{0.5,1.5,2,3\}$, and $r \in\{0, \pm 0.5\}$. Results are reported for sample sizes $n \in\{100,200,500\}$ and bandwidth settings $h=n^{-p}$ for $p \in\left\{\frac{1}{4}, \frac{1}{3}, \frac{1}{2.5}\right\}$. Note that $h=n^{-1 / 4}$ satisfies Assumption $2^{*}$ but not Assumption 2. The number of replications was 5, 000.

Table 1: Size: $\eta_{t}=\varepsilon_{t}$

\begin{tabular}{|c|c|c|c|c|c|c|}
\hline$r=0$ & \multicolumn{3}{|c|}{ Nominal Size $5 \%$} & \multicolumn{3}{|c|}{ Nominal Size 1\% } \\
\hline $\mathrm{n}$ & $h \quad n^{-\frac{1}{4}}$ & $n^{-\frac{1}{3}}$ & $n^{-\frac{1}{2.5}}$ & $n^{-\frac{1}{4}}$ & $n^{-\frac{1}{3}}$ & $n^{-\frac{1}{2.5}}$ \\
\hline 100 & 0.028 & 0.035 & 0.033 & 0.006 & 0.006 & 0.007 \\
\hline 200 & 0.034 & 0.042 & 0.041 & 0.007 & 0.007 & 0.008 \\
\hline 500 & 0.044 & 0.045 & 0.050 & 0.009 & 0.010 & 0.010 \\
\hline$r=0.5$ & & & & & & \\
\hline 100 & 0.030 & 0.035 & 0.040 & 0.006 & 0.007 & 0.007 \\
\hline 200 & 0.038 & 0.044 & 0.045 & 0.009 & 0.008 & 0.008 \\
\hline 500 & 0.041 & 0.045 & 0.048 & 0.008 & 0.009 & 0.009 \\
\hline$r=-0.5$ & & & & & & \\
\hline 100 & 0.031 & 0.035 & 0.037 & 0.007 & 0.008 & 0.008 \\
\hline 200 & 0.036 & 0.045 & 0.046 & 0.007 & 0.008 & 0.009 \\
\hline 500 & 0.041 & 0.047 & 0.051 & 0.009 & 0.010 & 0.011 \\
\hline
\end{tabular}

Table 2: Size: $\eta_{t}=\lambda \eta_{t-1}+\varepsilon_{t}, r= \pm 0.5$

\begin{tabular}{|c|c|c|c|c|c|c|}
\hline \multicolumn{4}{|c|}{ Nominal Size $5 \%$} & \multicolumn{3}{|c|}{ Nominal Size $1 \%$} \\
\hline \multicolumn{7}{|c|}{$r=0.5, \lambda=0.4$} \\
\hline & $n^{-\frac{1}{4}}$ & $n^{-\frac{1}{3}}$ & $n^{-\frac{1}{2.5}}$ & $n^{-\frac{1}{4}}$ & $n^{-\frac{1}{3}}$ & $n^{-\frac{1}{2.5}}$ \\
\hline 100 & 0.034 & 0.038 & 0.041 & 0.002 & 0.004 & 0.005 \\
\hline 200 & 0.044 & 0.044 & 0.047 & 0.004 & 0.006 & 0.007 \\
\hline 500 & 0.058 & 0.058 & 0.057 & 0.007 & 0.010 & 0.011 \\
\hline \multicolumn{7}{|c|}{$r=0.5, \lambda=-0.4$} \\
\hline 100 & 0.038 & 0.042 & 0.046 & 0.013 & 0.013 & 0.011 \\
\hline 200 & 0.051 & 0.051 & 0.051 & 0.018 & 0.015 & 0.014 \\
\hline 500 & 0.070 & 0.061 & 0.057 & 0.026 & 0.022 & 0.016 \\
\hline \multicolumn{7}{|c|}{$r=-0.5, \lambda=0.4$} \\
\hline 100 & 0.034 & 0.038 & 0.040 & 0.002 & 0.004 & 0.005 \\
\hline 200 & 0.044 & 0.044 & 0.048 & 0.004 & 0.006 & 0.007 \\
\hline 500 & 0.058 & 0.058 & 0.057 & 0.007 & 0.009 & 0.011 \\
\hline \multicolumn{7}{|c|}{$r=-0.5, \lambda=-0.4$} \\
\hline 100 & 0.035 & 0.040 & 0.043 & 0.012 & 0.012 & 0.012 \\
\hline 200 & 0.050 & 0.049 & 0.050 & 0.018 & 0.015 & 0.013 \\
\hline 500 & 0.073 & 0.064 & 0.056 & 0.026 & 0.018 & 0.016 \\
\hline
\end{tabular}


Table 2B: Size: $\eta_{t}=\varepsilon_{t}+\lambda \varepsilon_{t-1}, r= \pm 0.5$

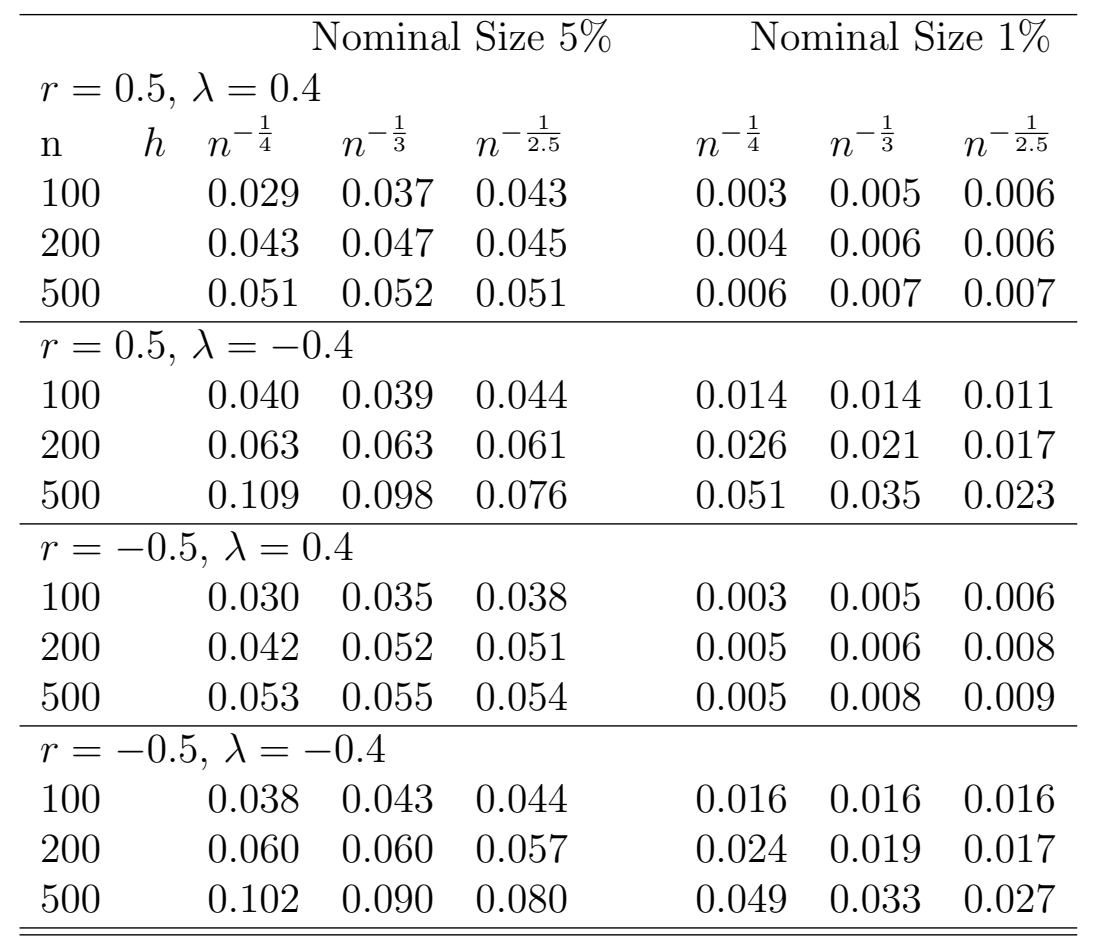


Table 3: Size: $\eta_{t}=\lambda \eta_{t-1}+\varepsilon_{t}, r= \pm 0.75$

\begin{tabular}{|c|c|c|c|c|c|c|}
\hline & & Jomina & Size $5 \%$ & & ninal S & ze $1 \%$ \\
\hline$r=$ & $\lambda=0$ & & & & & \\
\hline & $n^{-\frac{1}{4}}$ & $n^{-\frac{1}{3}}$ & $n^{-\frac{1}{2.5}}$ & $n^{-\frac{1}{4}}$ & $n^{-\frac{1}{3}}$ & $n^{-\frac{1}{2.5}}$ \\
\hline 100 & 0.036 & 0.038 & 0.039 & 0.003 & 0.003 & 0.004 \\
\hline 200 & 0.043 & 0.049 & 0.050 & 0.005 & 0.006 & 0.007 \\
\hline 500 & 0.057 & 0.055 & 0.053 & 0.007 & 0.009 & 0.008 \\
\hline$r=$ & $\lambda=$ & & & & & \\
\hline 100 & 0.074 & 0.068 & 0.027 & 0.036 & 0.033 & 0.027 \\
\hline 200 & 0.108 & 0.096 & 0.087 & .050 & 0.043 & 0.034 \\
\hline 500 & 0.177 & 0.140 & 0.115 & 0.094 & 0.062 & 0.048 \\
\hline$r=$ & $\lambda=0$ & & & & & \\
\hline 100 & 0.026 & 0.029 & 0.032 & 0.005 & 0.006 & 0.006 \\
\hline 200 & 0.037 & 0.044 & 0.046 & 0.007 & 0.008 & 0.010 \\
\hline 500 & 0.040 & 0.042 & 0.047 & 0.008 & 0.009 & 0.009 \\
\hline$r=$ & $75, \lambda=$ & & & & & \\
\hline 100 & 0.027 & 0.035 & 0.036 & 0.005 & 0.008 & 0.007 \\
\hline 200 & 0.036 & 0.040 & 0.043 & .008 & 0.010 & 0.010 \\
\hline 500 & 0.041 & 0.045 & 0.044 & 0.008 & 0.008 & 0.009 \\
\hline$r=$ & $75, \lambda=$ & & & & & \\
\hline 100 & 0.074 & 0.071 & 0.063 & 0.003 & 0.004 & 0.004 \\
\hline 200 & 0.103 & 0.085 & 0.074 & 0.011 & 0.012 & 0.011 \\
\hline 500 & 0.135 & 0.105 & 0.088 & 0.027 & 0.020 & 0.015 \\
\hline$r=$ & $75, \lambda=$ & -0.4 & & & & \\
\hline 100 & 0.070 & 0.066 & 0.065 & 0.033 & 0.026 & 0.023 \\
\hline 200 & 0.109 & 0.094 & 0.087 & 0.055 & 0.042 & 0.033 \\
\hline 500 & 0.175 & 0.136 & 0.109 & 0.093 & 0.065 & 0.048 \\
\hline
\end{tabular}


Table 3B: Size: $\eta_{t}=\varepsilon_{t}+\lambda \varepsilon_{t-1}, r= \pm 0.75$

\begin{tabular}{|c|c|c|c|c|c|c|}
\hline & & Nomina & Size $5 \%$ & Nol & ninal S & ze $1 \%$ \\
\hline$r=$ & $\lambda=0$ & & & & & \\
\hline & $n^{-\frac{1}{4}}$ & $n^{-\frac{1}{3}}$ & $n^{-\frac{1}{2.5}}$ & $n^{-\frac{1}{4}}$ & $n^{-\frac{1}{3}}$ & $n^{-\frac{1}{2.5}}$ \\
\hline 100 & 0.056 & 0.058 & 0.056 & 0.004 & 0.005 & 0.006 \\
\hline 200 & 0.075 & 0.070 & 0.064 & 0.006 & 0.007 & 0.008 \\
\hline 500 & 0.122 & 0.096 & 0.080 & 0.017 & 0.013 & 0.011 \\
\hline$r=$ & $\lambda=-$ & 0.4 & & & & \\
\hline 100 & 0.087 & 0.083 & 0.076 & 0.045 & 0.038 & 0.031 \\
\hline 200 & 0.176 & 0.142 & 0.119 & 0.095 & 0.069 & 0.055 \\
\hline 500 & 0.294 & 0.223 & 0.182 & 0.180 & 0.126 & 0.088 \\
\hline$r=$ & $\lambda=0$ & & & & & \\
\hline 100 & 0.033 & 0.037 & 0.043 & 0.006 & 0.007 & 0.008 \\
\hline 200 & 0.036 & 0.040 & 0.044 & 0.006 & 0.008 & 0.008 \\
\hline 500 & 0.041 & 0.043 & 0.045 & 0.008 & 0.009 & 0.010 \\
\hline$r=$ & $75, \lambda=$ & & & & & \\
\hline 100 & 0.034 & 0.038 & 0.041 & 0.006 & 0.009 & 0.007 \\
\hline 200 & 0.036 & 0.038 & 0.041 & 0.007 & 0.009 & 0.008 \\
\hline 500 & 0.039 & 0.045 & 0.047 & 0.009 & 0.009 & 0.009 \\
\hline$r=$ & $75, \lambda=$ & 0.4 & & & & \\
\hline 100 & 0.058 & 0.059 & 0.059 & 0.002 & 0.004 & 0.006 \\
\hline 200 & 0.080 & 0.072 & 0.067 & 0.008 & 0.009 & 0.010 \\
\hline 500 & 0.119 & 0.092 & 0.078 & 0.021 & 0.017 & 0.014 \\
\hline$r=$ & $75, \lambda=$ & -0.4 & & & & \\
\hline 100 & 0.038 & 0.043 & 0.044 & 0.016 & 0.016 & 0.016 \\
\hline 200 & 0.060 & 0.060 & 0.057 & 0.024 & 0.019 & 0.017 \\
\hline 500 & 0.102 & 0.090 & 0.080 & 0.049 & 0.033 & 0.027 \\
\hline
\end{tabular}


Table 4: Local Power: $\nu=3, \eta_{t}=\lambda \eta_{t-1}+\varepsilon_{t}, r= \pm 0.5$

\begin{tabular}{llllllll}
\hline \multicolumn{4}{c}{ Nominal Size $5 \%$} & \multicolumn{3}{c}{ Nominal Size $1 \%$} \\
$r=0.5, \lambda=0.4$ & & & & & \\
$\mathrm{n}$ & $h$ & $n^{-\frac{1}{4}}$ & $n^{-\frac{1}{3}}$ & $n^{-\frac{1}{2.5}}$ & $n^{-\frac{1}{4}}$ & $n^{-\frac{1}{3}}$ & $n^{-\frac{1}{2.5}}$ \\
100 & 0.819 & 0.779 & 0.743 & & 0.787 & 0.739 & 0.693 \\
200 & 0.906 & 0.878 & 0.845 & & 0.892 & 0.849 & 0.811 \\
500 & 0.971 & 0.950 & 0.923 & & 0.963 & 0.935 & 0.901 \\
\hline$r=0.5, \lambda=-0.4$ \\
100 & 0.247 & 0.211 & 0.179 & 0.197 & 0.154 & 0.126 \\
200 & 0.358 & 0.306 & 0.265 & 0.302 & 0.247 & 0.199 \\
500 & 0.522 & 0.448 & 0.389 & 0.458 & 0.376 & 0.310 \\
\hline$r=-0.5, \lambda=0.4$ \\
100 & 0.829 & 0.780 & 0.743 & 0.792 & 0.742 & 0.696 \\
200 & 0.910 & 0.879 & 0.845 & 0.891 & 0.851 & 0.813 \\
500 & 0.965 & 0.947 & 0.921 & 0.957 & 0.931 & 0.903 \\
\hline$r=-0.5, \lambda=-0.4$ \\
100 & 0.238 & 0.204 & 0.176 & 0.189 & 0.151 & 0.127 \\
200 & 0.352 & 0.297 & 0.253 & 0.295 & 0.239 & 0.193 \\
500 & 0.513 & 0.431 & 0.367 & 0.449 & 0.367 & 0.301 \\
\hline \hline
\end{tabular}

Table 4B: Local Power: $\nu=3, \eta_{t}=\varepsilon_{t}+\lambda \varepsilon_{t-1}, r= \pm 0.5$

\begin{tabular}{|c|c|c|c|c|c|c|}
\hline \multirow{2}{*}{\multicolumn{2}{|c|}{$r=0.5, \lambda=0$}} & \multicolumn{2}{|c|}{ Nominal Size 5\% } & \multicolumn{3}{|c|}{ Nominal Size $1 \%$} \\
\hline & & & & & & \\
\hline $\mathrm{n}$ & $n^{-\frac{1}{4}}$ & $n^{-\frac{1}{3}}$ & $n^{-\frac{1}{2.5}}$ & $n^{-\frac{1}{4}}$ & $n^{-\frac{1}{3}}$ & $n^{-\frac{1}{2.5}}$ \\
\hline 100 & 0.685 & 0.645 & 0.601 & 0.649 & 0.598 & 0.549 \\
\hline 200 & 0.800 & 0.751 & 0.719 & 0.769 & 0.715 & 0.664 \\
\hline 500 & 0.889 & 0.843 & 0.803 & 0.865 & 0.814 & 0.767 \\
\hline \multicolumn{7}{|c|}{$r=0.5, \lambda=-0.4$} \\
\hline 100 & 0.177 & 0.149 & 0.124 & 0.127 & 0.097 & 0.077 \\
\hline 200 & 0.279 & 0.232 & 0.200 & 0.223 & 0.175 & 0.139 \\
\hline 500 & 0.413 & 0.342 & 0.287 & 0.343 & 0.268 & 0.215 \\
\hline \multicolumn{7}{|c|}{$r=-0.5, \lambda=0.4$} \\
\hline 100 & 0.689 & 0.649 & 0.605 & 0.652 & 0.600 & 0.553 \\
\hline 200 & 0.788 & 0.739 & 0.699 & 0.757 & 0.703 & 0.649 \\
\hline 500 & 0.897 & 0.852 & 0.813 & 0.875 & 0.825 & 0.778 \\
\hline \multicolumn{7}{|c|}{$r=-0.5, \lambda=-0.4$} \\
\hline 100 & 0.142 & 0.121 & 0.109 & 0.104 & 0.082 & 0.066 \\
\hline 200 & 0.228 & 0.192 & 0.163 & 0.182 & 0.137 & 0.111 \\
\hline 500 & 0.375 & 0.310 & 0.254 & 0.309 & 0.238 & 0.186 \\
\hline
\end{tabular}


Table 5: Local Power: $\nu=2, \eta_{t}=\lambda \eta_{t-1}+\varepsilon_{t}, r= \pm 0.5$

\begin{tabular}{|c|c|c|c|c|c|c|}
\hline \multirow{2}{*}{\multicolumn{2}{|c|}{$r=0.5, \lambda=0}}$. & \multicolumn{2}{|c|}{ Nominal Size 5\% } & \multicolumn{3}{|c|}{ Nominal Size $1 \%$} \\
\hline & & & & & & \\
\hline $\mathrm{n}$ & $n^{-\frac{1}{4}}$ & $n^{-\frac{1}{3}}$ & $n^{-\frac{1}{2.5}}$ & $n^{-\frac{1}{4}}$ & $n^{-\frac{1}{3}}$ & $n^{-\frac{1}{2.5}}$ \\
\hline 100 & 0.357 & 0.282 & 0.228 & 0.282 & 0.205 & 0.147 \\
\hline 200 & 0.484 & 0.389 & 0.315 & 0.418 & 0.310 & 0.228 \\
\hline 500 & 0.682 & 0.557 & 0.458 & 0.616 & 0.482 & 0.376 \\
\hline \multicolumn{7}{|c|}{$r=0.5, \lambda=-0.4$} \\
\hline 100 & 0.058 & 0.054 & 0.053 & 0.027 & 0.020 & 0.016 \\
\hline 200 & 0.103 & 0.083 & 0.068 & 0.048 & 0.034 & 0.024 \\
\hline 500 & 0.169 & 0.118 & 0.094 & 0.098 & 0.057 & 0.036 \\
\hline \multicolumn{7}{|c|}{$r=-0.5, \lambda=0.4$} \\
\hline 100 & 0.114 & 0.123 & 0.128 & 0.065 & 0.066 & 0.067 \\
\hline 200 & 0.226 & 0.235 & 0.244 & 0.157 & 0.159 & 0.160 \\
\hline 500 & 0.437 & 0.457 & 0.462 & 0.350 & 0.359 & 0.367 \\
\hline \multicolumn{7}{|c|}{$r=-0.5, \lambda=-0.4$} \\
\hline 100 & 0.056 & 0.050 & 0.046 & 0.022 & 0.016 & 0.014 \\
\hline 200 & 0.102 & 0.082 & 0.066 & 0.053 & 0.031 & 0.022 \\
\hline 500 & 0.173 & 0.123 & 0.096 & 0.103 & 0.061 & 0.037 \\
\hline
\end{tabular}

Table 5B: Local Power: $\nu=2, \eta_{t}=\varepsilon_{t}+\lambda \varepsilon_{t-1}, r= \pm 0.5$

\begin{tabular}{llllllll}
\hline \multicolumn{4}{c}{ Nominal Size 5\% } & \multicolumn{3}{c}{ Nominal Size 1\% } \\
$\mathrm{n}=0.5, \lambda=0.4$ & $h$ & $n^{-\frac{1}{4}}$ & $n^{-\frac{1}{3}}$ & $n^{-\frac{1}{2.5}}$ & $n^{-\frac{1}{4}}$ & $n^{-\frac{1}{3}}$ & $n^{-\frac{1}{2.5}}$ \\
100 & 0.219 & 0.172 & 0.139 & 0.160 & 0.108 & 0.0 .76 \\
200 & 0.347 & 0.267 & 0.206 & 0.282 & 0.195 & 0.135 \\
500 & 0.513 & 0.390 & 0.304 & 0.437 & 0.318 & 0.220 \\
\hline$r=0.5$, & $\lambda=-0.4$ \\
100 & 0.050 & 0.049 & 0.050 & 0.019 & 0.016 & 0.013 \\
200 & 0.091 & 0.074 & 0.063 & 0.043 & 0.030 & 0.022 \\
500 & 0.164 & 0.116 & 0.092 & 0.088 & 0.051 & 0.035 \\
\hline$r=-0.5, \lambda=0.4$ \\
100 & 0.218 & 0.163 & 0.126 & 0.155 & 0.105 & 0.073 \\
200 & 0.343 & 0.260 & 0.201 & 0.267 & 0.180 & 0.126 \\
500 & 0.527 & 0.402 & 0.309 & 0.451 & 0.322 & 0.239 \\
\hline$r=-0.5, \lambda=-0.4$ \\
100 & 0.050 & 0.049 & 0.049 & 0.022 & 0.018 & 0.016 \\
200 & 0.088 & 0.074 & 0.064 & 0.042 & 0.033 & 0.022 \\
500 & 0.165 & 0.121 & 0.092 & 0.091 & 0.056 & 0.037 \\
\hline \hline
\end{tabular}


Table 6: Local Power: $\nu=1.5, \eta_{t}=\lambda \eta_{t-1}+\varepsilon_{t}, r= \pm 0.5$

\begin{tabular}{llllllll}
\hline \multicolumn{4}{c}{ Nominal Size 5\% } & \multicolumn{3}{c}{ Nominal Size $1 \%$} \\
$r=0.5, \lambda=0.4$ & & & & \\
$\mathrm{n}$ & $h$ & $n^{-\frac{1}{4}}$ & $n^{-\frac{1}{3}}$ & $n^{-\frac{1}{2.5}}$ & $n^{-\frac{1}{4}}$ & $n^{-\frac{1}{3}}$ & $n^{-\frac{1}{2.5}}$ \\
100 & 0.058 & 0.051 & 0.045 & & 0.021 & 0.012 & 0.010 \\
200 & 0.087 & 0.065 & 0.057 & & 0.040 & 0.022 & 0.015 \\
500 & 0.158 & 0.103 & 0.077 & 0.096 & 0.046 & 0.024 \\
\hline$r=0.5, \lambda=-0.4$ \\
100 & 0.043 & 0.040 & 0.041 & 0.016 & 0.014 & 0.012 \\
200 & 0.061 & 0.058 & 0.055 & 0.024 & 0.019 & 0.015 \\
500 & 0.096 & 0.074 & 0.070 & 0.038 & 0.031 & 0.023 \\
\hline$r=-0.5, \lambda=0.4$ \\
100 & 0.066 & 0.053 & 0.050 & 0.025 & 0.015 & 0.011 \\
200 & 0.093 & 0.065 & 0.052 & 0.046 & 0.023 & 0.015 \\
500 & 0.152 & 0.094 & 0.090 & 0.088 & 0.042 & 0.023 \\
\hline$r=-0.5, \lambda=-0.4$ \\
100 & 0.049 & 0.049 & 0.049 & 0.018 & 0.017 & 0.013 \\
200 & 0.063 & 0.058 & 0.059 & 0.024 & 0.021 & 0.017 \\
500 & 0.092 & 0.074 & 0.064 & 0.037 & 0.029 & 0.021 \\
\hline \hline
\end{tabular}

Table 6B: Local Power: $\nu=1.5, \eta_{t}=\varepsilon_{t}+\lambda \varepsilon_{t-1}, r= \pm 0.5$

\begin{tabular}{|c|c|c|c|c|c|c|}
\hline \multirow{2}{*}{\multicolumn{2}{|c|}{$r=0.5, \lambda=0}}$. & \multicolumn{2}{|c|}{ Nominal Size 5\% } & \multicolumn{3}{|c|}{ Nominal Size $1 \%$} \\
\hline & & & & & & \\
\hline $\mathrm{n}$ & $n^{-\frac{1}{4}}$ & $n^{-\frac{1}{3}}$ & $n^{-\frac{1}{2.5}}$ & $n^{-\frac{1}{4}}$ & $n^{-\frac{1}{3}}$ & $n^{-\frac{1}{2.5}}$ \\
\hline 100 & 0.181 & 0.193 & 0.198 & 0.112 & 0.117 & 0.115 \\
\hline 200 & 0.333 & 0.344 & 0.355 & 0.250 & 0.256 & 0.257 \\
\hline 500 & 0.577 & 0.591 & 0.596 & 0.489 & 0.500 & 0.505 \\
\hline \multicolumn{7}{|c|}{$r=0.5, \lambda=-0.4$} \\
\hline 100 & 0.054 & 0.058 & 0.061 & 0.022 & 0.021 & 0.019 \\
\hline 200 & 0.094 & 0.092 & 0.091 & 0.043 & 0.042 & 0.039 \\
\hline 500 & 0.188 & 0.175 & 0.164 & 0.109 & 0.096 & 0.089 \\
\hline \multicolumn{7}{|c|}{$r=-0.5, \lambda=0.4$} \\
\hline 100 & 0.114 & 0.123 & 0.128 & 0.065 & 0.066 & 0.067 \\
\hline 200 & 0.226 & 0.235 & 0.244 & 0.157 & 0.159 & 0.160 \\
\hline 500 & 0.437 & 0.457 & 0.462 & 0.350 & 0.359 & 0.367 \\
\hline \multicolumn{7}{|c|}{$r=-0.5, \lambda=-0.4$} \\
\hline 100 & 0.052 & 0.055 & 0.056 & 0.024 & 0.023 & 0.021 \\
\hline 200 & 0.096 & 0.089 & 0.086 & 0.043 & 0.038 & 0.037 \\
\hline 500 & 0.182 & 0.160 & 0.145 & 0.103 & 0.085 & 0.070 \\
\hline
\end{tabular}


Table 7: Local Power: $\nu=0.5, \eta_{t}=\lambda \eta_{t-1}+\varepsilon_{t}, r= \pm 0.5$

\begin{tabular}{llllllll}
\hline \multicolumn{4}{c}{ Nominal Size 5\% } & \multicolumn{3}{c}{ Nominal Size 1\% } \\
$r=0.5, \lambda=0.4$ & \multicolumn{3}{c}{$n^{-\frac{1}{4}}$} & $n^{-\frac{1}{3}}$ & $n^{-\frac{1}{2.5}}$ \\
$\mathrm{n}$ & $h$ & $n^{-\frac{1}{4}}$ & $n^{-\frac{1}{3}}$ & $n^{-\frac{1}{2.5}}$ & $n^{2.5}$ \\
100 & 0.035 & 0.040 & 0.042 & & 0.004 & 0.004 & 0.005 \\
200 & 0.040 & 0.043 & 0.046 & 0.007 & 0.007 & 0.008 \\
500 & 0.056 & 0.054 & 0.052 & 0.008 & 0.008 & 0.009 \\
\hline$r=0.5, \lambda=-0.4$ \\
100 & 0.039 & 0.045 & 0.048 & 0.014 & 0.013 & 0.012 \\
200 & 0.057 & 0.055 & 0.054 & 0.021 & 0.018 & 0.017 \\
500 & 0.080 & 0.068 & 0.059 & 0.032 & 0.023 & 0.018 \\
\hline$r=-0.5, \lambda=0.4$ \\
100 & 0.031 & 0.036 & 0.042 & 0.004 & 0.005 & 0.005 \\
200 & 0.043 & 0.048 & 0.050 & 0.005 & 0.006 & 0.007 \\
500 & 0.053 & 0.052 & 0.050 & 0.007 & 0.006 & 0.007 \\
\hline$r=-0.5, \lambda=-0.4$ \\
100 & 0.042 & 0.045 & 0.044 & 0.014 & 0.012 & 0.010 \\
200 & 0.050 & 0.052 & 0.053 & 0.018 & 0.016 & 0.014 \\
500 & 0.080 & 0.068 & 0.060 & 0.032 & 0.024 & 0.017 \\
\hline \hline
\end{tabular}

Table 7B: Local Power: $\nu=0.5, \eta_{t}=\varepsilon_{t}+\lambda \varepsilon_{t-1}, r= \pm 0.5$

\begin{tabular}{llllllll}
\hline \multicolumn{4}{c}{ Nominal Size $5 \%$} & \multicolumn{3}{c}{ Nominal Size 1\% } \\
$r=0.5, \lambda=0.4$ & & & & & \\
$\mathrm{n}$ & $h$ & $n^{-\frac{1}{4}}$ & $n^{-\frac{1}{3}}$ & $n^{-\frac{1}{2.5}}$ & $n^{-\frac{1}{4}}$ & $n^{-\frac{1}{3}}$ & $n^{-\frac{1}{2.5}}$ \\
100 & 0.028 & 0.034 & 0.036 & & 0.002 & 0.004 & 0.003 \\
200 & 0.043 & 0.048 & 0.049 & & 0.005 & 0.007 & 0.008 \\
500 & 0.050 & 0.052 & 0.053 & & 0.008 & 0.009 & 0.010 \\
\hline$r=0.5, \lambda=-0.4$ \\
100 & 0.045 & 0.049 & 0.053 & 0.020 & 0.019 & 0.017 \\
200 & 0.065 & 0.057 & 0.055 & 0.028 & 0.021 & 0.017 \\
500 & 0.102 & 0.088 & 0.074 & 0.047 & 0.032 & 0.024 \\
\hline$r=-0.5, \lambda=0.4$ \\
100 & 0.029 & 0.040 & 0.047 & 0.003 & 0.002 & 0.005 \\
200 & 0.041 & 0.048 & 0.046 & 0.003 & 0.005 & 0.006 \\
500 & 0.049 & 0.052 & 0.054 & 0.006 & 0.008 & 0.009 \\
\hline$r=-0.5, \lambda=-0.4$ & & & & \\
100 & 0.047 & 0.048 & 0.048 & 0.019 & 0.017 & 0.016 \\
200 & 0.070 & 0.065 & 0.062 & 0.028 & 0.023 & 0.018 \\
500 & 0.107 & 0.093 & 0.079 & 0.049 & 0.036 & 0.028 \\
\hline \hline
\end{tabular}

